\title{
Review and Catalog of the Ostracode Family Rutidermatidae (Crustacea: Myodocopa)
}

\author{
Elizabeth Harrison-Nelson, ${ }^{1}$ John M. Orr, ${ }^{2}$ Margaret E. Tarmann, ${ }^{1}$ and Louis S. Kornicker ${ }^{1}$ \\ ${ }^{1}$ Department of Invertebrate Zoology, National Museum of Natural History, Smithsonian Institution, Washington, \\ DC 20013-7012, USA \\ ${ }^{2}$ Department of Environmental Science and Policy, George Mason University, 4400 University Drive, MSN 5F2, \\ Fairfax, VA 22030-4444, USA \\ Correspondence should be addressed to Louis S. Kornicker, kornickl@si.edu \\ Received 31 January 2011; Accepted 17 March 2011 \\ Academic Editors: A. Arslan and V. Ketmaier
}

Copyright ( $) 2011$ Elizabeth Harrison-Nelson et al. This is an open access article distributed under the Creative Commons Attribution License, which permits unrestricted use, distribution, and reproduction in any medium, provided the original work is properly cited.

Cohen and Kornicker (1987) presented a catalog of species of the Rutidermatidae that had been published prior to March 1986. The present contribution includes one additional subfamily, one additional genus, and twelve additional species (one new) and, also, keys to subfamilies, genera, and species, illustrations of carapaces, tables giving characteristics of appendages, and maps showing distribution of species.

\section{Introduction}

Family Rutidermatidae belongs to the subclass Myodocopa, order Myodocopida. Order Myodocopida can further be divided into the three suborders Cladocopina, Halocypridina, and Myodocopina. The Rutidermatidae is one of five extant families included in the ostracod suborder Myodocopina. It contains two subfamilies: the Rutidermatinae with three genera (Alternochelata with four species, Rutiderma with 37 species, and Scleraner with two species) and the Metaschismatinae with one genus (Metaschisma with two species). Cohen and Kornicker [1] published a catalog of species of the Rutidermatidae that had been reported prior to March 1986. Since then, an additional subfamily and genus as well as 12 new species have been included in the family, and taxa have been reported from additional localities. We have updated the catalog to August 2009 and have included keys and appendices to help compare and identify the species in this family. Keys are used with care; determination of species should always be verified by use of the descriptions and figures. A tabular key to families of Myodocopida was given in Cohen and Morin [2, Appendix 3] and in Cohen et al. [3, Table 4].

\section{Class Ostracoda Latreille 1806}

Superorder Myodocopa Sars 1866
Order Myodocopida Sars 1866

Suborder Myodocopina Sars 1866

Family Rutidermatidae Brady and Norman 1866

Composition. The Rutidermatidae includes two subfamilies, the Rutidermatinae (with three genera, Alternochelata, Rutiderma, and Scleraner) and the Metaschismatinae (with one genus, Metaschisma).

Description. Carapace oval in lateral view with male more elongate than female; surface punctate or smooth; prominent ribbing absent in Scleraner, present in most species of Rutiderma; projecting caudal process in some species, absent in others; rostrum generally rounded anteriorly; incisur minute in some species and forming small indentation creating overhanging rostrum in others; rostrum of adult male more prominent than that of adult female and juveniles.

First antenna: The third and fourth segments fused in females of some species, separated by suture in adult male; fifth segment long in female and juveniles, small in adult male; sensory bristle of adult female either bare or with few short filaments, of adult male with broad proximal part with numerous long slender filaments; sixth segment of juveniles and adult female minute and fused with fifth segment, of 
male long and with suture separating it from fourth and fifth segments; a-bristle of seventh segment not claw-like; $\mathrm{d}$ - and e-bristles of eighth segment about same length, bare with blunt tips; c-bristle of seventh segment and f-bristle of eighth segment of female about same length as d- and ebristles, of adult male extremely long and with numerous short filaments.

Second antenna: Protopodite without distal medial bristle; endopodite of female small with 1 segment in Rutiderma and 2 in Scleraner, of male 3-segmented and reflexed in both genera; exopodite of female with natatory hairs on long bristles of segments 6-9, of male on long bristles of segments 3-9, of juveniles without natatory hairs on bristles.

Mandible with long narrow hirsute exopodite on male, rudimentary or absent on female; second endopodial segment of female broad with stout ventral claw forming pincer with stout claw of third endopodial segment, of adult male second endopodial segment narrow without stout claw, and with long narrow claw on third endopodial segment.

Maxilla of female stout with 3 endites; exopodial segments of male with bristles; female with stout teeth on first exopodial segment, and broad flat tooth on second exopodial segment. Second thoracic leg well-developed in both sexes but with fewer bristles on male; terminus with opposing combs.

Furca of Rutiderma with 3 or 4 stout claws followed by 2 or 3 secondary claws, of Scleraner with 4 stout claws followed by 5 secondary claws, of Alternochelata with secondary claws between main claws.

Bellonci organ elongate, 1 or 2 segmented, with rounded or pointed tip. Lateral eyes of female small with 4 or 5 ommatidia, of male larger with 14-30 ommatidia.

Medial eye large, pigmented, with dorsal filaments on some species of Rutiderma.

Copulatory organ of adult male elongate terminating in small lobes bearing minute processes and bristles (Kornicker [4, page 171$])$.

Biology. Juveniles and adult females are carnivores eating mostly crustaceans and worms; guts of adult males generally are empty or have little food. Members of the family have 4 juvenile instars; carapaces of instars of both sexes are similar and generally resemble that of the adult female. The adult male is a more efficient swimmer than the female and is occasionally collected in the water column without females; it is generally sparser than the female in collections from substrate; juveniles of both sexes, which are without natatory hairs on the exopodial bristles of the second antenna, are restricted to the bottom (Kornicker [4, page 171]).

Distribution. Members of the family are widespread between latitudes of $45^{\circ} \mathrm{N}$ and $53^{\circ} \mathrm{S}$ at mostly shelf depths (0-200 m), but they also have been collected on the upper slope (200$1100 \mathrm{~m}$ ). Rutiderma and Scleraner have been collected in the Subantarctic and are absent in the Antarctic (Kornicker [4, page 171]). Tables 1-4 list Rutidermatidae distribution by hemisphere, ocean, locations and depths, and water temperatures and salinities, for those species for which data are available.
TABLE 1: Rutidermatidae distribution by hemisphere.

\begin{tabular}{ll}
\hline Northern Hemisphere & Southern Hemisphere \\
\hline A. nealei & A. lizardensis \\
A. polychelata & M. nex \\
A. sikorai & R. arx \\
R. apex & R. dux \\
R. arcuatilis & R. exrex \\
R. chessi & R. ferax \\
R. cohenae & R. gerdhartmanni \\
R. compressa & R. ovate \\
R. darbyi & R. rex \\
R. dinochelata & R. sagax \\
R. flex & R. tryx \\
R. fusca & S. chacaoi \\
R. gyre & S. trifax \\
R. hartmanni & \\
R. irrostrata & \\
R. judayi & \\
R. leloeuffi & \\
R. licina & \\
R. lomae & \\
R. kalkei & \\
R. mollitum & \\
R. mortenseni & \\
R. normani & \\
R. pax & \\
R. rostrata & \\
R. rotunda & \\
R. schroederi & \\
R. sterreri & \\
R. tridens & \\
R. vox & \\
\hline
\end{tabular}

Comparisons. Species are mapped in Figure 10 (Alternochelata, Scleraner, and Metaschisma), and Figures 11, 12 and 13 (Rutiderma). The Rutidermatidae is the only family having mandibles of juveniles and adult females with a stout claw on the second endopodial segment forming a pincer with the stout claw of the third endopodial segment. A stout claw is absent on the second endopodial segment of the mandible of the adult male (Kornicker [4, page 171]).

Ecology. Some abiotic variables that influence the distribution of living marine ostracods are substrate, temperature and salinity, depth, abundance and seasonality.

Klie [5, pages 404, 406 (substrate)], Kornicker [6, pages 195, 202, 217, 222, 223-224, 236-238 (response to light, substrate, temperature, salinity)], Hartmann [7, page 39 (substrate)], Hartmann-Schröder in HartmannSchröder and Hartmann [8, pages 30, 31 (substrate)], McKenzie [9, pages 58-60 (substrate, temperature, salinity, productivity)], Poulsen [10, pages 22, 31, 35 (substrate)], Lie [11, pages 274, 288, 550 (substrate)], Lie and Kisker 
TABLE 2: Rutidermatidae distribution by ocean.

\begin{tabular}{|c|c|c|}
\hline Atlantic Ocean & Pacific Ocean & Indian Ocean \\
\hline A. sikorai & A. lizardensis & R. $\operatorname{arx}$ \\
\hline A. nealei & M. nex & R. $d u x$ \\
\hline A. polychelata & R. apex & R. exrex \\
\hline R. darbyi & R. chessi & R. ferax \\
\hline R. arcuatilis & R. $d u x$ & R. fusca \\
\hline R. cohenae & R. gerdhartmanni & R. rex \\
\hline R. compressa & R. hartmanni & R. sagax \\
\hline R. dinochelata & R. judayi & \\
\hline R. flex & R. lomae & \\
\hline R. gyre & R. normani & \\
\hline R. hartmanni & R. ovate & \\
\hline R. irrostrata & R. pax & \\
\hline R. kalkei & R. rostrata & \\
\hline R. leloeuffi & R. rotunda & \\
\hline R. licina & R. $\operatorname{try} x$ & \\
\hline R. mollitum & R. vox & \\
\hline R. mortenseni & S. chacaoi & \\
\hline R. schroederi & S. trifax & \\
\hline \multicolumn{3}{|l|}{ R. sterreri } \\
\hline R. tridens & & \\
\hline
\end{tabular}

[12, page 2279 (substrate, community)], Kornicker [13, pages 25, 29, 30, 37 (substrate, temperature)], Kornicker and Meyers [14, pages 2, 4, 10, 19, 25 (substrate, temperature)], Kornicker [15, page 793 (substrate, salinity)], Kornicker $[16$, pages $4,7,11,12,24,70,85$ (substrate, temperature, salinity)].

Substrate. The Rutidermatidae has been collected on a variety of substrates including sand, shelly sand, shell, gravel, ooze, eel grass in tide pools, corals, oyster shells, red sponge, algae, clay, silty clay, plankton, mud, muddy sand, Gorgonacea, and under rocks [1] but are most commonly found on a sand substrate, which may facilitate burrowing. Grabe et al. [17] found that the densities of eight species of myodocopid ostracods excluding rutidermatids were positively correlated with a silt-clay substrate but the density of $R$. darbyi was not.

Temperature and Salinity. Rutidermatidae has been collected in water temperatures ranging from $9.8^{\circ}$ to $32^{\circ} \mathrm{C}$, salinities from 30 to $42 \%$ [ [1], and dissolved oxygen levels from 2.5 to $9.2 \mathrm{mg} / 1$ [18]. When collected from Tampa Bay, $R$. darbyi was more abundant at greater depth, in coarser sediment, and in more saline water than was average for the bay suggesting that $R$. darbyi enters the bay from the Gulf of Mexico [18].

Depth. The Rutidermatidae has been collected as deep as $560 \mathrm{~m}$. Deeper records exist but they are questionable [1]. Alternochelata nealei was purportedly collected from
$1100 \mathrm{~m}$ but the sample contained species of Cyclasteropinae and Asteropteroninae that were confined to continental shelf depths in the area [19]. Kornicker [13] reported a single $R$. ovata collected at $1834 \mathrm{~m}$ but the sample contained many cypris larvae and euphausiids which were inconsistent with the reported method of collection.

Biology: Reproduction. Rutidermatids are usually confined to the continental shelf $(0-200 \mathrm{~m}$ depth) but are occasionally collected on the upper continental slope $(200-1100 \mathrm{~m}$ depth). Males and females may be collected at different depths. In some species, females become completely benthic after mating by breaking bristles off the second antenna [16], whereas males typically remain free-swimming. Deep water may pose a barrier to their distribution as they are generally not collected around isolated island systems or Antarctica which has a deep continental shelf. Cohen [20] found that rutidermatids were abundant at a shallow lagoon site but uncommon at a deeper fore-reef site. Grabe et al. [17], however, collected fewer $R$. darbyi from shallow subtidal stations than from deeper stations offshore [21, pages 184$185,196,199]$.

Abundance. Large concentrations of rutidermatids have been found in a specific area. For example, rutidermatids were the most abundant family of ostracods collected in Belize [20], where $R$. dinochelata was the most abundant species, accounting for $32.4 \%$ of the myodocopids collected from a lagoon. $R$. darbyi was the second most abundant ostracod collected in Tampa Bay, accounting for $19.8 \%$ of the ostracods sampled [18]. $R$. darbyi and R. mollitum were the third and fourth most numerous ostracod species in samples taken off Florida [22].

Seasonality. In Florida, the abundance of $R$. darbyi was correlated with the seasons [17]. Horsley [22] found the abundance of myodocopid ostracods, including rutidermatids, was greater in May and June (193 rutidermatids sampled) than in December (100 rutidermatids sampled).

\section{Catalog of the Rutidermatidae}

General Works. Skogsberg [23, pages 31, 33, 156, 159, 165, 166, 168, 171, 173, 195], Mertens [24, Plates 6, 10], Poulsen [25, pages $5,11,13,339,365]$, Poulsen [10, pages 5-18, 47-50, 52, 56, 146, 147, 155, 451-454, 457, 464, 465, 468, 481-483, Figure 152, Tables 2, 3] Hartmann [26, pages 95, $166,168,176,183,184-185,193,200,207-209]$, Hartmann and Puri [27, page 14], Hartmann [28, pages 671 (key to the suborder Myodocopa), 681 (diagnosis)], Kornicker [13, pages $34,35,37,38,41,42-43,46,48,52,53,83-84,645-$ 646, Tables 13, 15, 16, 17, 18, Figures 4, 9, 10, 11, 12, 15, 17, 22, 23], Kornicker and Cohen [29, Table 1], Kornicker [30, pages $1,30-36,42,43,46-49,52,53,55-57,63,66$, 67, Tables 8, 15], Kornicker [31, page 5], Kornicker and Meyers [14, pages 1, 2], Kornicker [16, pages 1, 12, 16, Table 1], Kornicker [19, page 2], Kornicker [32, pages 174178, Figure 113], Kornicker [33, Table 3], Maddocks et al. [34, page 282], Cohen and Kornicker [1, pages 1-8], Hartmann and Petersen [35, pages 157, 158], Cohenand Morin 
TABle 3: Rutidermatidae species locations and depths.

\begin{tabular}{|c|c|c|}
\hline & Location & Depth $(\mathrm{m})$ \\
\hline \multicolumn{3}{|l|}{ Western Atlantic Region } \\
\hline Alternochelata polychelata & Bahamas; Belize & $1-5$ \\
\hline Alternochelata sikorai & Gulf of Mexico & $61-137.2$ \\
\hline Rutiderma arcuatilis & Virgin Islands & 9 \\
\hline Rutiderma cohenae & Key West; Bahamas; Belize & Subtidal-4 \\
\hline Rutiderma darbyi & East coast USA; Gulf of Mexico; Belize; Bahamas & Intertidal-168 \\
\hline Rutiderma dinochelata & East coast USA; Bahamas; Belize & Intertidal-20 \\
\hline Rutiderma flex & Bahamas & 25 \\
\hline Rutiderma gyre & Florida Shelf; Gulf of Mexico & $6.1-148$ \\
\hline Rutiderma hartmanni & Belize & $1.5,18-30$ \\
\hline Rutiderma kalkei & Gulf of Mexico & 91 \\
\hline Rutiderma. licina & East coast USA; Gulf of Mexico & $17-68$ \\
\hline Rutiderma mollitum & East coast USA; Gulf of Mexico & $5.4-190$ \\
\hline Rutiderma mortenseni & Virgin Islands & $?$ \\
\hline Rutiderma schroederi & Bahamas & $67,90-100$ \\
\hline Rutiderma sterreri & Bermuda & Intertidal-11 \\
\hline \multicolumn{3}{|l|}{ Eastern Atlantic Region } \\
\hline Alternochelata nealei & West coast Africa & $53-560,1100 ?$ \\
\hline Rutiderma compressa & West coast Europe & 150 \\
\hline Rutiderma irrostrata & Mauritania & $94-250$ \\
\hline Rutiderma. leloeuffi & Ivory Coast; Mauritania & $20-150$ \\
\hline Rutiderma tridens & Mauritania & 30 \\
\hline \multicolumn{3}{|l|}{ Indo-West Pacific Region } \\
\hline Alternochelata lizardensis & Lizard Island Group, Australia & Intertidal-12.3 \\
\hline Metaschisma nex & New South Wales, Australia & 204 \\
\hline Rutiderma arx & Madagascar; Comoros; Mozambique & Reef flat-31 \\
\hline Rutiderma dux & Darwin, Australia; Lizard Island, Australia & Intertidal \\
\hline Rutiderma exrex & Madagascar & Infralittoral-27 \\
\hline Rutiderma ferax & Madagascar & Residual pool \\
\hline Rutiderma fusca & Red Sea & Surface \\
\hline Rutiderma normani & Thailand, Philippines & $2-17$ \\
\hline Rutiderma rex & Comoros & 26 \\
\hline Rutiderma sagax & Darwin, Australia & Intertidal \\
\hline Rutiderma tryx & Lizard Island, Australia & 1.5 \\
\hline Rutiderma vox & Enewetak Atoll, Marshall Islands & $3-7$ \\
\hline Scleraner trifax & New South Wales, Australia & $204-400$ \\
\hline \multicolumn{3}{|l|}{ East Pacific Region } \\
\hline Rutiderma apex & California & $1.8-5.2,9.1-11$ \\
\hline Rutiderma chessi & San Clemente Island, California & $?$ \\
\hline Rutiderma gerdhartmanni & Chile & 12 \\
\hline Rutiderma hartmanni & Pearl Islands, Panama & 9 \\
\hline Rutiderma judayi & California; Baja California, Mexico & $7-21$ \\
\hline Rutiderma lomae & California & Surface \\
\hline Rutiderma ovata & Chile & $176-192,1834 ?$ \\
\hline Rutiderma pax & El Salvador & 12 \\
\hline Rutiderma rostrata & California; Baja California, Mexico & $11-22$ \\
\hline Rutiderma rotunda & California; Baja California, Mexico & $0.3-22$ \\
\hline Scleraner chacaoi & Chile & 190 \\
\hline
\end{tabular}


TABLE 4: Rutidermatidae water temperatures and salinities.

\begin{tabular}{lcc}
\hline Species & Temperature $\left({ }^{\circ} \mathrm{C}\right)$ & Salinity \\
\hline Alternochelata polychelata & $\sim 29$ & 37 \\
Rutiderma dinochelata & $\sim 29$ & $31-42$ \\
Rutiderma judayi & $18.5-25$ & $34-37$ \\
Rutiderma leloeuffi & 28.9 & 34.4 \\
Rutiderma lomae & 9.8 & \\
Rutiderma mollitum & 32 & 30,35 \\
Rutiderma rotunda & 18.5 & $34-37$ \\
Scleraner chacaoi & $\sim 11$ & \\
\hline
\end{tabular}

[21, pages 184, 185, 195-196, 199, Table 2], Morin et al. [36, pages 3-4, Table 1], Kornicker [37, pages 114, 190, 192 (continental shelf of Australia)], Parker [38, pages 633, 650], Parker [39, pages 95, 104], Kornicker [40, pages 680-681], Kornicker [41, pages 268, 270-272], Kornicker [42, pages 798, 800, 808, Figure 11A], Kornicker and Harrison-Nelson [43, pages 426-428, 455-456, 460, 465, 467, Figure 1, Tables 1, 7-9], Kornicker et al. [44, page 82, Table 1], Cohen in Cohen et al. [3, page 432, Table 4, Plate 187: B,C, Plate 188: A,B], Frame et al. [45, page 337].

Keys. Müller [46, pages 34, 35 (Rutiderma)], Poulsen [10, pages 17-18 (Rutiderma)], Kornicker [13, Key to Families: pages 83, 646 (south of latitude $35^{\circ} \mathrm{S}$ )], Kornicker and Caraion [47, page 54], Kornicker and Cohen [29, page 500], Kornicker and Myers [14, pages 3, 4 (Southern California coast)], Kornicker [16, pages 16, 25 (genera worldwide, species of SE North America and Gulf of Mexico)], Cohen et al. [3, Table 4], Cohen and Morin [2, Appendix 3].

Regional Works. Continental Shelf Southeastern North America, northern half Gulf of Mexico, Bahama Islands, Andros Island, Bimini Islands, U.S. Virgin Islands, Windward Islands, Barbados, West Indies and Bermuda, Kornicker [16, pages 1-89], Bermuda, Maddocks et al. [34, pages 280288], Northwest Pacific Ocean: Marshall Islands-Enewetak and Bikini Atolls, Kornicker [48, pages 78-84], Indian Ocean: NE Mozambique Channel, Kornicker [49, 123-144], Darwin, Northern Territory, Australia Lizard Island, Great Barrier Reef, Palfrey Island (Lizard Island Group), Queensland, Australia, Kornicker [50, pages 93-94], North Pacific, Pillar Point Harbor, Half Moon Bay, California, Kornicker and HarrisonNelson [51, pages 36-45], Tuléar Reef Complex, SW Madagascar, Kornicker and Thomassin [52, pages 73-92], Gulf of Mexico: Florida-Tampa Bay, Grabe [18, page 62, Tables 2-5], Harrison-Nelson et al. [53, pages 873-874], Scripps Coastal Reserve and Cortez Street in La Jolla, California, Frame et al. [45, page 332].

Biology: Reproduction. Cohen and Morin [21, pages 184185, 196, 199].

Parasites. Choniostomatid copepods found in Rutiderma darbyi and R. sterreri.

Life history and ontogeny. Key to instars of Rutiderma darbyi Kornicker and Harrison-Nelson [43, page 456].

\section{Key to the Genera of Rutidermatidae (Adapted from Kornicker and Caraion page 54 of [47])}

1. Furca with secondary claws alternating with primary (Alternochelata)

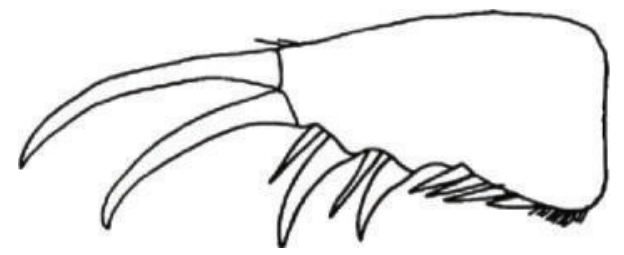

Furca with secondary claws following primary claws

(2)

Furca with some claws alternating and some not. (See also Table 9) (Metaschisma)

2. Endopodite of female second antenna with 1 segment (Rutiderma)

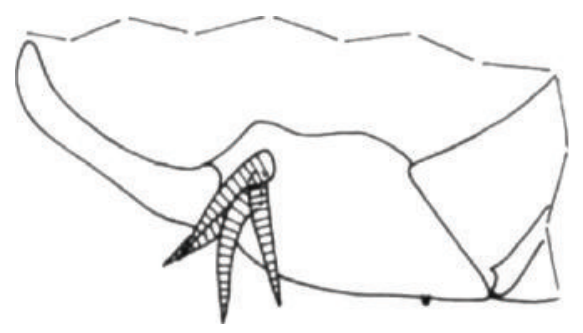

Endopodite of female second antenna with 2 segments. (See also Table 9) (Scleraner)

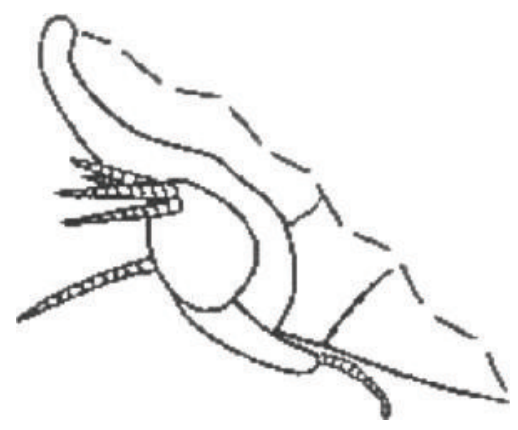

3.1. Alternochelata Kornicker [6]. Rutiderma (Alternochelata) Kornicker [6, pages 236-238] (type-species, by monotypy: Rutiderma (Alternochelata) polychelata Kornicker [6]; gender: feminine), Poulsen [10, pages 11, 17], Hartmann and Puri [27, page 14 (mentions)].

Alternochelata. McKenzie [9, page 62], Hartmann in Hartmann-Schröder and Hartmann [8, page 328], Hartmann [28, page 681], Kornicker [13, pages 645, 646, 678], Kornicker [54, page 40 (mentions)], Kornicker and Caraion [47, page 66 (keys)], Kornicker and Meyers [14, page 2], Kornicker [16, pages 12, 16 (Key to species of Rutiderma), Table 1], Cohen and Kornicker [1, page 2], Kornicker [37, pages 114, 123], Kornicker and Harrison-Nelson [43, pages $465,467]$. 
Distribution. Atlantic: Great Bahama Bank; northern Gulf of Mexico; off Mauritania and Western Sahara. Pacific: Great Barrier Reef, Australia; coast of Chile.

Habitat. Marine, benthic, from 1-5600 m (questionable record at $1100 \mathrm{~m}$; Kornicker [16, page 16]) on substrates of sand, muddy sand, mud, and Gorgonacea.

Life History and Ontogeny. From 1 to 6 brooded eggs, broken swimming bristles on adult females of $A$. sikorai, $A$. nealei, and A. lizardensis.

\section{Key to the Species of Alternochelata Kornicker [6]}

(See also Tables 5 and 6).

1. Carapace with rounded posteroventral corner; each lamella of furca with main 1, 2, 3, 5

Carapace with angular posteroventral corner; each lamella of furca with main claws $1,2,4,5$, or $1,2,4,6$ (3)

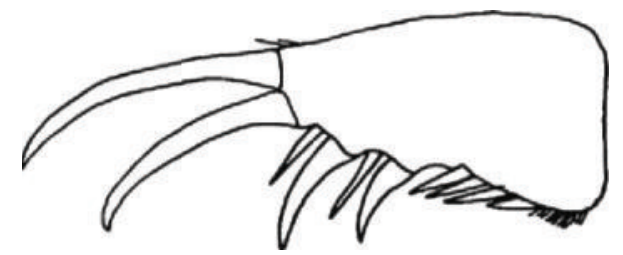

2. Fossae on surface of carapace bordered by reticulations formed by minute but distinct pustules (A. sikorai)

Fossae on surface of carapace not bordered by reticulations formed by minute pustules (A. nealei)

3. Each lamella of furca with main claws $1,2,4,5 \quad$ (A. lizardensis)

Each lamella of furca with main claws $1,2,4,6 \quad$ (A. polychelata)

\subsection{Rutidermatinae Brady and Norman [55]}

4.1.1. Alternochelata lizardensis Kornicker [15] (Figures 1(a)1(c)). Alternochelata lizardensis Kornicker [15, pages 793805, Figures 1-6], Kornicker [16, pages 12, 16, (key), 25], Cohen and Kornicker [1, page 2], Kornicker and HarrisonNelson [43, page 467, Tables 8, 9].

Holotype. USNM 158609, adult female.

Type Locality. Lizard Island main lagoon, undisturbed sand flat, depth $6 \mathrm{~m}$, Great Barrier Reef, Australia.

Distribution. North Atlantic, viz. the Great Bahama Bank (Kornicker [6, page 237]), off Mauritania (Kornicker and Caraion [47, page 66]), and Lizard Island Group, Great Barrier Reef, Australia.

Habitat. Benthic; from low intertidal to $12.3 \mathrm{~m}$; sand flat near coral reef.

Life History and Ontogeny. Adult male and female, A-1 male, 4 to 6 eggs, broken swimming bristles on females.
Comparisons. Alternochelata lizardensis differs from $A$. nealei in that the carapace bears a distinct caudal process; also, furcal claws $1,2,3,5$ of $A$. nealei are primary claws, whereas claw 3 is a secondary claw on the furca of $A$. lizardensis. The furca of $A$. lizardensis does not have a secondary claw between 2 sets of primary claws as on $A$. polychelata (Kornicker [15, page 805]).

4.1.2. Alternochelata nealei Kornicker and Caraion [47] (Figures 1(d)-1(e)). Alternochelata nealei Kornicker and Caraion [47, pages 3-6, 66-73, Table 1, Figures 1-3, 56-59], Kornicker and Myers [14, page 2 (mentions)], Kornicker [16, pages 12, 16 (key), 24, 25], Cohen and Kornicker [1, page 2], Kornicker and Harrison-Nelson [43, pages 467, Tables 8, 9].

Alternochelata neali Kornicker [15, page 805 (compares to A. lizardensis; misspelling)].

Holotype. "Grigore Antipa" 293, Female, Museum of Natural History, Bucharest, Romania.

Type Locality. Station X013, $19^{\circ} 46^{\prime} 00^{\prime \prime} \mathrm{N}, 17^{\circ} 08^{\prime} 00^{\prime \prime} \mathrm{W}$; $61 \mathrm{~m}$; Islamic Republic of Mauritania.

Distribution. Northeast Atlantic off Mauritania and Western Sahara.

Habitat. Benthic, shelf-bathyal, from 53 to 560 m (questionable record at $1100 \mathrm{~m}$ ); mud, muddy sand, Gorgonacea washings, and sand substrates.

Life History and Ontogeny. Adult male and female, from two to three eggs, female with broken natatory bristles.

Comparisons. The species $A$. nealei differs from $A$. polychelata, Kornicker [6], in not having a distinct caudal process on the carapace. Also, the main claws of the furca are claws 1-3, 5 on A. nealei and claws 1, 2, 4, 6 on A. polychelata (Kornicker and Caraion [47, page 73]).

4.1.3. Alternochelata polychelata (Kornicker [6]) (Figure 1(f)). Rutiderma (Alternochelata) polychelata Kornicker [6, pages 232, 237, 238, Figures 46:6A-B, 59A-E, 86C-G], Poulsen [10, pages 7, 8, 17, 18, 43], Cohen [20, page 326], McKenzie [9, page 62 (inferred)], Kornicker and Caraion [47, pages 66, 73 (compares to A. nealei)], Kornicker [15, pages 793, 805 (compares to A. lizardensis)], Kornicker [16, pages 11-14, 16 (key), 17, 25, Table 1, Figures 1, 5].

Rutiderma polychelata. Hartmann in HartmannSchröder and Hartmann [8, page 328 (mentions)] .

Alternochelata polychelata. Kornicker [33, Table 3], Kornicker [41, 42, Table 1], Kornicker and Harrison-Nelson [43, page 467, Tables 8, 9], Kornicker et al. [56, Table 3].

Holotype. USNM 122908.

Type Locality. Station 110F-2, Bimini Islands, Bahama Islands.

Distribution. Bimini, Bahamas; Caribbean Sea, Belize.

Habitat. Benthic; from 1 to $5 \mathrm{~m}$; calcareous sand, mangroves (Avicennia); about $29^{\circ} \mathrm{C}$, about $37 \%$ o salinity.

Life History and Ontogeny. Sex of holotype and paratype unknown.

Comparisons. Alternochelata polychelata differs from $A$. nealei in having a distinct caudal process on the carapace, and while, on $A$. nealei, the main claws of the furca are 13 , and 5 , on $A$. polychelata, the main claws are $1,2,4$, and 
TABLE 5: Summary of characteristics of mature females in Alternochelata.

\begin{tabular}{|c|c|c|c|c|}
\hline Species & lizardensis & nealei & polychelata & sikorai \\
\hline Shell length, mm & 1.36 & $1.18 \mathrm{~mm}$ & 1.37 & 1.27 \\
\hline Surface with ribs (r) or smooth (s) & s & s & s & s \\
\hline Incisur shallow or deep & ${ }^{*}$ Deep & ${ }^{*}$ Deep & *Deep & *Deep \\
\hline No. of medial br. on rostrum & $(11-12)$ & 7 & $?$ & 6 \\
\hline \multicolumn{5}{|l|}{ 1st antenna } \\
\hline no. of br. 2nd seg., d-la & $1-1-0$ & $1-1-1$ & $?$ & $1-1-1$ \\
\hline no. of br. 3rd seg., d.-v & $1-1$ & $1-1$ & $?$ & $1-1$ \\
\hline no. of br. 4 th seg., d.-v & $1-3$ & $1-3$ & $?$ & $1-3$ \\
\hline no. of fil. on sens. br. & 3 & 1 & $?$ & 1 \\
\hline no. of fil. on b.-br & 1 & 0 & $?$ & 0 \\
\hline no. of fil. on c.-br. & 3 & 1 & $?$ & 1 \\
\hline no. of fil. on g.-br. & 3 & 1 & $?$ & 1 \\
\hline \multicolumn{5}{|l|}{ 2nd antenna } \\
\hline endop. no. of br. & 6 & 5 & $(5-6)$ & 5 \\
\hline endop. no. of br. on 2 nd seg. & 2 & 1 & $?$ & 1 \\
\hline endop. no. of br. on 3 rd seg. & 0 , when present & abs. & $?$ & abs. \\
\hline \multicolumn{5}{|l|}{ Mandible } \\
\hline coxale + or - bifurcate endite & + & + & $?$ & + \\
\hline basale, no. of d. br. mid.-dist. & 3 & 3 & 4 & 3 \\
\hline basale, no. of thin v. br. & $* 3$ & $* 3$ & ? & $*_{5}$ \\
\hline basale, no. of stout v. br. & $* 3$ & $* 3$ & ? & $* 2$ \\
\hline basale, total no. of v. br. & 6 & 6 & 7 & 7 \\
\hline 1st end. seg., no. of v. br. & 3 & 2 & 2 & 3 \\
\hline 2nd end. seg., no. of d. br. & 7 & $(5-6)$ & 5 & 7 \\
\hline 2nd end. seg., no. of v. br. excl. claw & 2 & 2 & 4 & 2 \\
\hline 3rd end. seg., no. of br. excl. claw & 5 & 5 & 3 & 5 \\
\hline Maxilla, no. of br. on the 3 endites & $3-2-4$ & $7-4-(5-6)$ & $?$ & $8-5-7$ \\
\hline \multicolumn{5}{|l|}{5 th limb } \\
\hline no. of br. on the 3 endites & $2-4-8$ & $2-3-4$ & $?$ & $3-5-5$ \\
\hline ex. seg., no. of primary teeth & 2 & 3 & $?$ & 3 \\
\hline 2nd ex. seg., no. of teeth & 2 & 3 & $?$ & 3 \\
\hline 2nd ex. seg., + or - bi-or trifurcate teeth & + & + & $?$ & + \\
\hline 3rd ex. seg., no. of br. on each lobe & $2-2$ & $3-2$ & $?$ & $3-2$ \\
\hline 4 th +5 th segs. no. of br. & 5 & $(3-4)$ & $?$ & $(2-4)$ \\
\hline \multicolumn{5}{|l|}{ 6th limb } \\
\hline no. of epipodial br. & 2 & 2 & $?$ & $(2-3)$ \\
\hline no. of br. endites & $3-3-4-3$ & $3-3-3-2$ & $?$ & $3-3-3-2$ \\
\hline end-seg., no. of br. & 8 & 7 & $?$ & $(6-7)$ \\
\hline \multicolumn{5}{|l|}{ 7th limb } \\
\hline no. of terminal cleaning br. & $3-3$ & $3-3$ & $3-3$ & $3-3$ \\
\hline no. of prox. cleaning br. & $2-2$ & $2-2$ & $2-2$ & $(1-2)-2$ \\
\hline no. of comb-teeth & $9-3$ & $11-0$ & $2-5$ & $14-0$ \\
\hline Furca, no. of main claws & 4 & 4 & 4 & 4 \\
\hline Furca, no. of secondary claws & 6 & 6 & 2 & 6 \\
\hline Lateral eyes, + or - & + & - & + & - \\
\hline Specimen Number, \# = USNM & \#158488 & \#141269 & \#122908 & \#158027 \\
\hline
\end{tabular}

abs.: absent; br.: bristles; d: dorsal; dist.: distal; endop.: endopodite; ex.: exopod; fil.: filaments; no.: number; prox.: proximal; seg.: segment; sens: sensory; ?: unknown; v: ventral, ${ }^{*}$ : after Poulsen [10]. 
TABLE 6: Summary of characteristics of Alternochelata male.

\begin{tabular}{|c|c|c|c|}
\hline Species & lizardensis & nealei & sikorai \\
\hline Shell length, mm & 1.36 & 1.38 & 1.38 \\
\hline Surface with ribs (r) or smooth (s) & s & s & s \\
\hline Incisur shallow or deep & ${ }^{*}$ Deep & *Deep & *Deep \\
\hline \multicolumn{4}{|l|}{ No. medial br. rostrum } \\
\hline (rostral infold) & $?$ & 7 & 6 \\
\hline \multicolumn{4}{|l|}{ 1st antenna } \\
\hline no. br. 2nd seg., d.-l.-v. & $1-1$ & $1-1-1$ & $1-1-1$ \\
\hline no. br. 3rd seg., d.-v & $1-1$ & $1-1$ & $1-1$ \\
\hline no. br. 4th seg., d.-v & $1-4$ & $1-4$ & $1-4$ \\
\hline no. fil. sens. br. & Numerous & 33 & Numerous \\
\hline no. fil. b.-br & 3 & 3 & 3 \\
\hline no. fil. c.-br. & 13 & 12 & 13 \\
\hline no. fil. g.-br. & 3 & $(1-2)$ & 1 \\
\hline \multicolumn{4}{|l|}{ 2nd antenna } \\
\hline endop. no. br. 1st seg. & 6 & 6 & 6 \\
\hline endop. no. br. 2nd seg. & 2 & 2 & 2 \\
\hline endop. no. br. 3rd seg. & 3 & 2 & 2 \\
\hline \multicolumn{4}{|l|}{ Mandible } \\
\hline \multicolumn{4}{|l|}{ Basale } \\
\hline no. d. br. mid.-dist. & 3 & 3 & 3 \\
\hline no. thin v. br. & $* 6$ & $?$ & $* 5$ \\
\hline no. stout v. br. & $* 0$ & $?$ & $* 3$ \\
\hline total no. v. br. & 6 & 8 & 8 \\
\hline exop. + or - & - & + & - \\
\hline 1st end. seg., no. v. br. & 3 & 3 & 3 \\
\hline 2nd end. seg., no. d. br. & 7 & 7 & 7 \\
\hline 2nd end. seg., no. v. br. excl. cl. & 6 & 6 & 6 \\
\hline 3rd end. seg., no. br. excl. cl. & 6 & 5 & 6 \\
\hline Maxilla, no. br. 3 endites & $6-4-4$ & $6-6-7$ & $(5-6)-4-6$ \\
\hline \multicolumn{4}{|l|}{5 th $\operatorname{limb}$} \\
\hline no. br. 3 endites & $3-4-7$ & $2-(2-3)-(4-5)$ & $(2-4)-4-5$ \\
\hline 3rd exop. seg., no. br. each lobe & $3-2$ & $2-2$ & $2-2$ \\
\hline 5th limb, 4th +5 th segs. no. br. & 5 & 4 & $(3-4)$ \\
\hline \multicolumn{4}{|l|}{ 6th limb } \\
\hline no. epipodial br. & 2 & 2 & 2 \\
\hline no. br. 4 endites & $(3-4)-(3-4)-4-3$ & $2-3-3-2$ & $3-3-3-(1-2)$ \\
\hline end.-seg., no. br. & 9 & 7 & 6 \\
\hline \multicolumn{4}{|l|}{ 7th limb } \\
\hline no. terminal cleaning br. & $2-2$ & $2-2$ & $2-2$ \\
\hline no. prox. cleaning br. & $2-2$ & $2-2$ & $(1-2)-2$ \\
\hline no. comb-teeth & $9-2$ & $(11-13)-0$ & $13-0$ \\
\hline \multicolumn{4}{|l|}{ Furca } \\
\hline no. main claws & 4 & 4 & 4 \\
\hline no. secondary claws & 5 & 6 & 6 \\
\hline Copulatory limbs long or short (sh.) & sh. & $*$ sh. & $?$ \\
\hline USNM Specimen Number & \#158487 & \#156596 & \#158935 \\
\hline
\end{tabular}

br.: bristles; cl.: claw; d.: dorsal; dist.: distal; endop.: endopodite; excl.: excluding; exop.: exopodite; fil.: filaments; 1.: lateral; no.: number; prox.: proximal; seg.: segment; sens: sensory; v.: ventral. * after Poulsen [10]. 


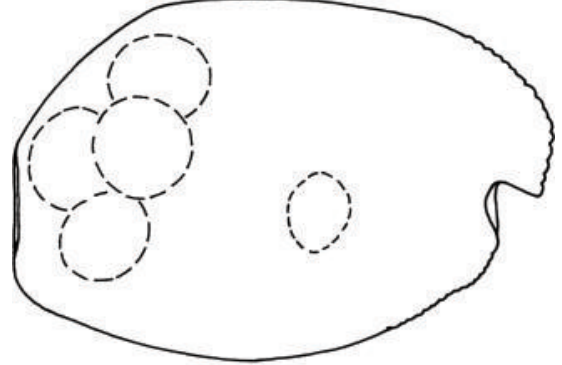

(a)

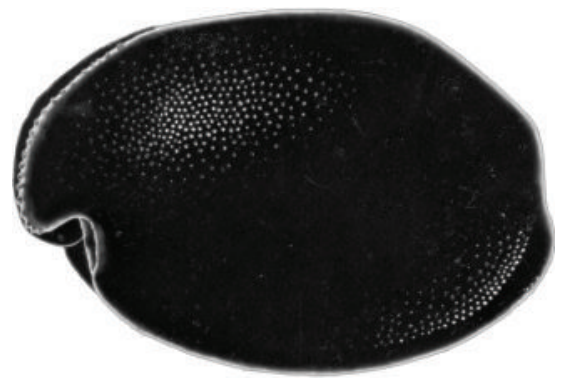

(c)

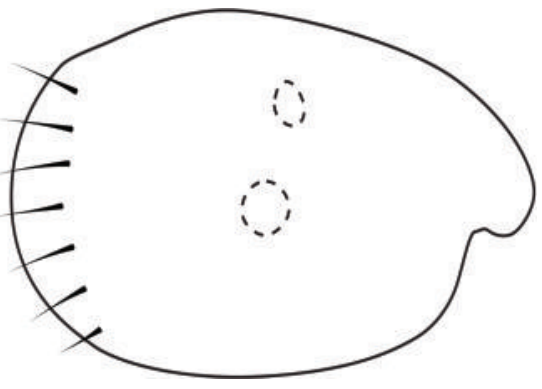

(e)

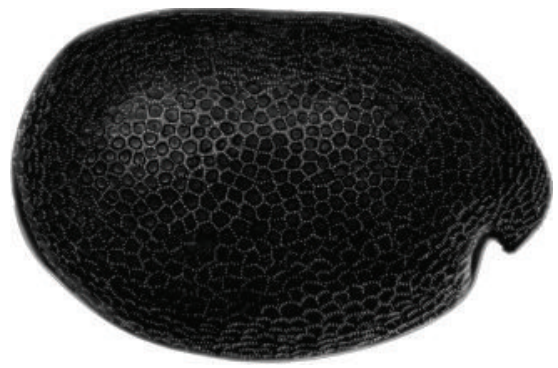

(g)

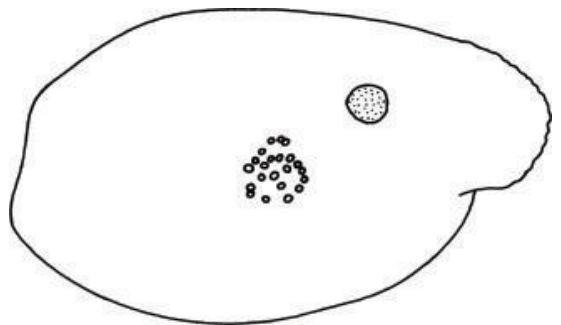

(b)

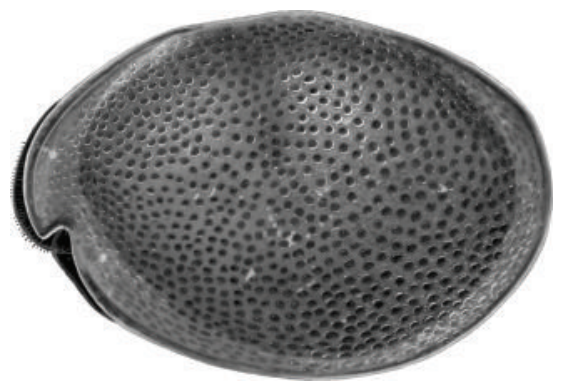

(d)

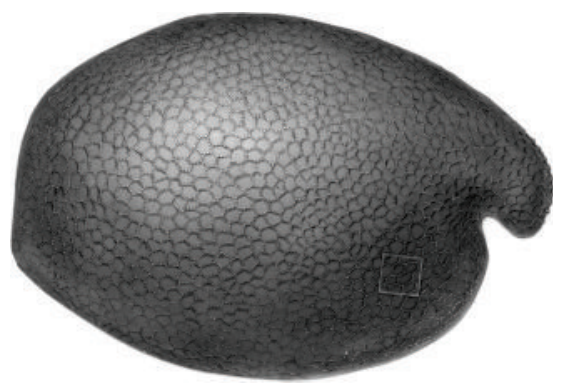

(f)

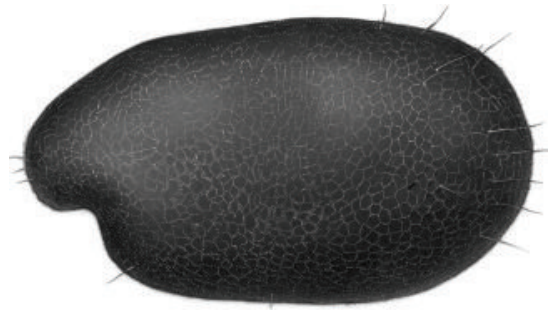

(h)

Figure 1: (a) Alternochelata lizardensis, ovigerous female, $1.43 \mathrm{~mm}$. (b) A. lizardensis, male, $1.36 \mathrm{~mm}$. (c) A. lizardensis, A-1 male, $1.29 \mathrm{~mm}$. (d) A. nealei, female, $1.18 \mathrm{~mm}$. (e) A. nealei, male, $1.27 \mathrm{~mm}$. (f) A. polychelata, female, $1.37 \mathrm{~mm}$. (g) A. sikorai, female, $1.27 \mathrm{~mm}$. (h) A. sikorai, male, $1.38 \mathrm{~mm}$.

6. Furcal claw distribution also distinguishes $A$. polychelata from A. lizardensis in that $A$. polychelata has secondary claws between two sets of primary claws. Alternochelata polychelata also has a more rounded posteroventral corner of the shell than A. sikorai.

4.1.4. Alternochelata sikorai Kornicker [16] (Figure 1(g)1(h)). Alternochelata sikorai Kornicker [16, pages 1, 7-10, 12-14, 16 (key), 17-25, Table 1, Figures 1 (distribution), 69], Kornicker [33, Table 3], Kornicker and Harrison-Nelson
[43, page 467, Tables 8, 9], Harrison-Nelson and Kornicker [53, page 874$]$.

Alternochelata species A. Flint [57, Figure 4].

Holotype. USNM 158027, adult female.

Type Locality. Station EFG-2, in vicinity of East Flower Garden, continental shelf off Texas, $27^{\circ} 53^{\prime} \mathrm{N}, 93^{\circ} 38^{\prime} \mathrm{W}$, depth $112 \mathrm{~m}$.

Distribution. Gulf of Mexico, off Louisiana and Texas.

Habitat. Benthic; from 61 to $137.2 \mathrm{~m}$. 
Life History and Ontogeny. Adult male and female, 1 to 3 eggs; broken swimming bristles on female.

Comparisons. The species $A$. sikorai is closely related to A. nealei, having the claws of the furca similarly distributed and the carapace having a similar shape. It differs from that species in having the reticulations of the carapace being formed by rows of minute but distinct pustules. The distribution of furcal claws differs from that of $A$. polychelata, and the posteroventral corner of the shell is more rounded (Kornicker [16, pages 24-25]).

4.2. Metaschismatinae Kornicker [37]. Metaschismatinae Kornicker [37, page 123], Kornicker and Harrison-Nelson [43, page 428].

Diagnosis and Description. Kornicker [37, page 123].

Distribution. Off Nowra, New South Wales, Australia, depth $204 \mathrm{~m}$.

General Habitat. Benthic; upper continental slope (Kornicker [37, Appendix 1]).

4.2.1. Metaschisma Kornicker [37]. Metaschisma Kornicker [37, page 123 (monotypic; type-species, Metaschisma nex)], Kornicker and Harrison-Nelson [43, pages 465, 467].

Distribution. Off Nowra, New South Wales, Australia.

Habitat. Marine; benthic; $204 \mathrm{~m}$, on substrate of coarse shell.

Life History and Ontogeny. From 3 to 6 brooded eggs, broken swimming bristles on adult females of $M$. nex. Instar A-1 male with short bristles without natatory hairs on exopod of second antenna.

4.2.2. Metaschisma nex Kornicker [37] (Figure 2(a)). Metaschisma nex Kornicker [37, pages 123-128, 192, Figures 6971, 111b, Tables 1, 2, Appendices 1, 2], Kornicker and Harrison-Nelson [43, page 467, Tables 1, 8].

Holotype. NMV J35600, ovigerous female, in collection of Museum of Victoria, Abbottsford, Australia.

Type Locality. Slope 1, 34 ${ }^{\circ} 59.52^{\prime} \mathrm{S}, 151^{\circ} 5.94^{\prime} \mathrm{E}$, off Nowra, New South Wales, $204 \mathrm{~m}$.

Distribution. Type locality only.

Habitat. Upper slope, 204 m; coarse shell.

Life History and Ontogeny. Adult female, A-1 male, late juveniles; ovigerous females with 3 to 6 eggs; swimming bristles of adult female broken, juveniles with short swimming bristles without natatory hairs.

\subsection{Rutidermatinae Brady and Norman [55]}

4.3.1. Rutiderma Brady and Norman [55]. Rutiderma Brady and Norman [55, pages 623, 627, 640-642, 673] (type species, by monotypy: Brady and Norman [55, gender neuter]), Juday [58, page 147 (diagnosis)], Müller [59, pages 90-92 (diagnosis, compares to other genera)], Müller [46, pages 24, 34, 35 (diagnosis, key)], Skogsberg [23, pages 165-168, 173 (systematic position)], Kornicker [6, pages 236, 237 (diagnosis of subgenus Rutiderma)], Hartmann et al. [60, page 328 (compares to Alternochelata)], McKenzie
[9, pages 57, 62 (diagnosis, compares to Alternochelata)], Poulsen [10, pages 6-17 (diagnosis, general), keys to species of males, females: pages 17, 18, (key) 22, 38, 43 (map), 52, 453, 456, Figures 1b, c, 13 (maps of distribution of species), 151c, 152B], Hartmann [26, pages 95, 129, 165, 183, 184], Hartmann [28, pages 588 (found in the Red Sea), 589 (in sand and mixed substrate), 591 (found in Chile), 681], Kornicker [13, pages 645-648, 678 (diagnosis, compares to other genera, Key to Genera, Key to Antarctic Species)], Kornicker [54, page 40], Kornicker [31, pages 1, 5], Kornicker and Myers [14, pages 1, 2 (diagnosis, keys to Southern California species, adult females and males)], Bonaduce et al. [61, page 474, (Red Sea) Figure 2(4)], Kornicker [16, pages $1,12,16,25$, Table 1, Key (Key to species of western North Atlantic and Gulf of Mexico)], McCain [62, page 99 Appendix 1 (Arabian Gulf)], Kornicker [63, pages 1, 3, 24, 25, 26 (Bay of Panama, Pacific Ocean)], Kornicker [33, (western North Atlantic and Gulf of Mexico), Table 3], Kornicker [49, page 123 (Indian Ocean)], Kornicker [37, pages 114, 123 (southeastern Australian continental shelf)], Parker [39, page 105], Kornicker and Harrison-Nelson [43, pages 426, 428, 429, 465, 467], Oakley and Cunningham [64, Figure 1 (mentions)], Oakley [65, page 184].

Type Species. Rutiderma compressa Brady and Norman [55, page 673].

Distribution. This genus is cosmopolitan between the latitudes of $45^{\circ} \mathrm{N}$ and $53^{\circ} \mathrm{S}$, at depths from intertidal to $317 \mathrm{~m}$ (questionably to $1834 \mathrm{~m}$ ), and contains about 37 species (Cohen and Kornicker [1, page 2], Kornicker [49, page 123], Kornicker [50, page 22]).

Life History and Ontogeny. Key to Instars I-IV, male, female (Kornicker [49, page 123]).

\section{Key to Species of Rutiderma (Females)}

See also Tables 3, 4, 7, 8 (males), 10, 11 (juveniles).

1. Surface of valves smooth

(R. $\operatorname{ar} x)$

Surface of valves with ribs

2. Second segment of the first antenna with 1 bristle

Second segment of the first antenna with 2 bristles

3. Carapace length approximately

$1.1 \mathrm{~mm}$

Carapace length around $1.5 \mathrm{~mm}$ or greater

4. Incisur shallow

(R. compressa)

Incisur deep

(R. schroederi)

5. Third segment of the first antenna with 2 bristles

Third segment of the first antenna with 3 bristles

6. Lateral eye present

Lateral eye absent

(R. irrostrata) 
7. Sensory bristle of first antenna with 1 filament

Sensory bristle of first antenna lacking filaments

8. Sensory bristle of first antenna with no filaments

Sensory bristle of first antenna with filaments

9. Furca with 4 primary claws and 2 secondary claws

Furca with 3 primary claws and 3 secondary claws

10. Second endopodial segment of mandible with 4 dorsal bristles

Second endopodial segment of mandible with 3 dorsal bristles

11. Lateral eyes absent

Lateral eyes present

12. Sixth limb with 2 bristles instead of

epipodial appendage

Sixth limb with 1 bristle instead of epipodial appendage

13. Carapace length approximately $1 \mathrm{~mm}$ Carapace length approximately $1.46 \mathrm{~mm}$

14. Carapace length slightly less than $1 \mathrm{~mm}$

Carapace length much greater than

$1 \mathrm{~mm}$, around $1.3 \mathrm{~mm}$

15. Furca with 4 primary claws and 2 secondary claws

Furca with 3 primary claws and 3 secondary claws

16. Carapace length less that $1 \mathrm{~mm}$

Carapace length well above $1 \mathrm{~mm}$

17. Incisur shallow and barely noticeable Incisur pronounced and deep

18. Rostral infold with 7 bristles

Rostral infold with 8-12 bristles

19. Seventh limb with a total of 7 comb teeth

Seventh limb with around 20 comb teeth 20 C-bristle on first antenna bare, with no filaments

C-bristle on first antenna with 1 filament

21. Incisur shallow

Incisur deep

22. Rostral infold with 10 bristles

Rostral infold with less than 10 bristles

23. Sensory bristle on first antenna with 3 bristles

Sensory bristle on first antenna with 1 bristle

24. C-bristle on first antenna bare
(R. sterreri) C-bristle on first antenna with 2

filaments

(R. arcuatilis)

25. Sensory bristle with 5 filaments

(R. cohenae)

(R. leloeuffi) Sensory bristle with only 1 or 2 filaments

26. Exterior of carapace with bristles on

(9) the ventral and anterodorsal margins

Exterior of carapace with no bristles

27. Fifth limb with 5 bristles on the

fourth and fifth segments

(R. $d u x)$

(R. dinochelata) Fifth limb with 3 or 4 bristles on the

fourth and fifth segments

(10) 28. C-bristle of first antenna with 1

filament

(R. normani) C-bristle of first antenna lacking

filaments

(R. licina) 29. Rostrum and incisur with sharp angle and sharp tip

(R. pax)

(R. lomae)

(R. vox)

(R. rostrata)

(R. rex)

(R. rotunda)

(R. mollita)

Rostrum and incisur with a smooth rounded angle and rounded tip

30. Carapace has very pronounced horizontal ribs with high relief

Carapace with less pronounced horizontal ribs

31. C-bristle of first antenna lacks

filaments

C-bristle of first antenna with 1 filament

32. List of caudal process of left valve

serrate along anterior and ventral

margins

List of caudal process of left valve not

serrate.

\subsection{Rutiderma apex Kornicker and Harrison-Nelson}

$$
\text { [51] (Figures 2(b)-2(c)) }
$$

5.1.1. Rutiderma sp. Tuel et al. page 155 of [66] . Rutiderma apex. Kornicker and Harrison-Nelson [51, pages 4, 36-45, Figures 2, 6, 7, 19-25 (compares to other species), Tables 13], Kornicker and Harrison-Nelson [43, Tables 1, 8, 9], Oakley [67, Figure 1 (mentions)], Oakley [65, Figures 1, 2, 3, Tables 1,2], Cohen et al. [3, page 432, Plate 187: B,C, Plate 188: A, B].

Holotype. USNM 158263, ovigerous female.

(R. ovata)

(R. gerdhartmanni)

(R. ferax)

(R. exrex)

(R. chessi)

(R. apex)
Type Locality. Pillar Point Harbor, Half Moon Bay, California, Station 9A (Dec).

Distribution. California coast: Stations 5-9, Pillar Point Harbor, Half Moon Bay, depth range from 1.8 to $5.2 \mathrm{~m}$; Dark Gulch, $39^{\circ} 14.5^{\prime} \mathrm{N}, 123^{\circ} 45.8^{\prime} \mathrm{W}$, Mendocino County, depth from 9.1 to $11 \mathrm{~m}$; Tomales Bay, near Spud Pt. Marina, Bodega Harbor.

Habitat. Clayey silt and sand.

Life History and Ontogeny. Adult male, A-1 male, and female, from 3 to 4 eggs.

Comparisons. The surface ornamentation of $R$. apex resembles that of $R$. judayi McKenzie [9], except that the female is without a small process near the middle of posterior margin that projects past the posterior end of the valve. The length of the female carapace of R. judayi is from 0.95 
TABLE 7: Summary of characteristics of mature females in Rutiderma.

(a)

\begin{tabular}{|c|c|c|c|c|c|c|c|}
\hline Species & apex & arcuatilis & $\operatorname{arx}$ & chessi & cohenae & compressa & darbyi \\
\hline Shell length, mm & 1.18 & 1.02 & 1.43 & 1.21 & 1.24 & 1.6 & 1.28 \\
\hline Surface with ribs (r) or smooth (s) & $\mathrm{r}$ & $\mathrm{r}$ & $\mathrm{s}$ & $\mathrm{r}$ & $\mathrm{r}$ & $\mathrm{r}$ & $\mathrm{r}$ \\
\hline Incisur shallow or deep & $*$ Deep & $*$ Deep & *Shallow & $*$ Deep & $*$ Deep & * Shallow & ${ }^{*}$ Deep \\
\hline No. of medial br. on rostrum (infold) & 10 & 10 & $7-8$ & 10 & $7-8$ & $?$ & 7 \\
\hline \multicolumn{8}{|l|}{ 1st antenna } \\
\hline no. of br. 2nd seg., d-la & $1-1$ & $1-1$ & $1-1$ & $1-1$ & $1-1$ & $1-0$ & $1-1$ \\
\hline no. of br. 3rd seg., d.-v & $2-1$ & $2-1$ & $2-1$ & $2-1$ & $2-1$ & $?$ & $2-1$ \\
\hline no. of br. 4 th seg., d.-v & $1-2$ & $1-2$ & $1-2$ & $1-2$ & $1-2$ & $1-2$ & $1-2$ \\
\hline no. of fil. on sens. br. & 1 & 1 & 2 & 3 & 5 & ? & 2 \\
\hline no. of fil. on b.-br & 0 & 0 & 0 & 0 & 0 & ? & 0 \\
\hline no. of fil. on c.-br. & 0 & 2 & 1 & 1 & 2 & $?$ & 2 \\
\hline no. of fil. on g.-br. & 1 & 2 & 2 & 1 & 3 & $?$ & 1 \\
\hline \multicolumn{8}{|l|}{ 2nd antenna } \\
\hline endop. no. of br. & 4 & 4 & 5 & 4 & 5 & 4 & 4 \\
\hline \multicolumn{8}{|l|}{ Mandible } \\
\hline coxale + or - bifurcate endite & + & + & + & + & + & + & + \\
\hline basale, no. of d. br. mid.-dist. & 3 & 3 & 3 & 3 & 3 & $?$ & 3 \\
\hline basale, no. of thin v. br. & 2 & 4 & 3 & $* 3$ & 5 & $?$ & $* 5$ \\
\hline basale, no. of stout v. br. & 4 & 3 & 4 & $* 3$ & 2 & $?$ & $* 2$ \\
\hline basale, total no. of v. br. & 6 & 7 & 7 & 6 & 7 & $?$ & 7 \\
\hline 1st end. seg., no. v. br. & 2 & 2 & 2 & 2 & 2 & $?$ & 2 \\
\hline 2nd end. seg., no. of d. br. & 3 & 3 & 4 & 3 & 4 & $?$ & 3 \\
\hline 2nd end. seg., no. of v. br. excl cl.. & 5 & 1 or 2 & 5 & 2 or 3 & 2 & $?$ & 3 \\
\hline 3rd end. seg., no. of br. excl. cl. & 4 & 6 & 3 & 6 & 6 & $?$ & 6 \\
\hline Maxilla, no. br. on the 3 endites & $2-3-4$ & $? \quad(2$ & $(2-4)-(2-4)-(3-5)$ & $(2-3)-(2-3)-(2-3)$ & ) $6-4-8$ & $?$ & $6-5-6$ \\
\hline \multicolumn{8}{|l|}{ Fifth limb } \\
\hline no. br. on the 3 endites & $2-6-(6-7)$ & 15 & $3-5-7$ & $3-5-7$ & $5-6-10$ & $?$ & $(3-4)-5-(5-6)$ \\
\hline ex. seg., no. primary teeth & 4 & 4 & $*_{4}$ & $* 4$ & $* 4$ & 4 & 4 \\
\hline 2nd ex. seg., no. teeth & 3 & 2 & $* 3$ & $* 3$ & $* 3$ & 3 & $* 3$ \\
\hline 2nd ex. seg., + or - bi-or trifurcate teeth & + & + & + & + & + & $?$ & + \\
\hline 3rd ex. seg., no. br. on each lobe & $2-2$ & $3-2$ & $3-2$ & $3-2$ & $2-2$ & $?$ & $2-3$ \\
\hline 4 th +5 th segs. no. br. & 5 & 5 & 4 & 4 & 4 & 4 & 4 \\
\hline \multicolumn{8}{|l|}{ Sixth limb } \\
\hline no. epipodial br. & 2 & 2 & 2 & 1 or 2 & 2 & 2 & 2 \\
\hline no. br. on the 4 endites & $3-2-2-2$ & $3-3-2-3$ & $(2-3)-2-3-(3-4)$ & $3-2-2-2$ & $3-3-(2-4)-3$ & $2-2-3-2$ & $3-2-2-3$ \\
\hline end-seg., no. br. & 7 & 7 & 6 & 7 & 7 & 7 & 7 \\
\hline \multicolumn{8}{|l|}{7 th $\operatorname{limb}$} \\
\hline no. terminal cleaning br. & $3-3$ & $3-3$ & $3-3$ & $3-3$ & $3-3$ & $3-3$ & $3-3$ \\
\hline no. prox. cleaning br. & $2-2$ & $2-2$ & $2-2$ & $2-2$ & $2-2$ & $2-2$ & $2-2$ \\
\hline no. comb-teeth & $6-6$ & $5-4$ & $4-(5-6)$ & $5-3$ & $3-5$ & $1-5$ & $10-4$ \\
\hline Furca, no. of main claws & 4 & 4 & 3 & 4 & 4 & 3 & 4 \\
\hline Furca, no. of secondary claws & 2 & 2 & 3 & 2 & 2 & 3 & 2 \\
\hline Lateral Eyes, + or - & + & + & + & + & + & + & + \\
\hline Specimen Number, \# = USNM & \#158262 & \#158212 & \#193411 & $\# 158280$ & $\# 158414$ & $*$ & $\# 158628 \mathrm{~A}$ \\
\hline
\end{tabular}


(b)

\begin{tabular}{|c|c|c|c|c|c|c|c|}
\hline Species & dinochelata & $d u x$ & exrex & ferax & gerdhartmanni & gyre & hartmannii \\
\hline Shell length, mm & 1.18 & 0.99 & 0.99 & 1.16 & 1.36 & 1.1 & 1.12 \\
\hline Surface with ribs (r) or smooth (s) & $\mathrm{r}$ & $\mathrm{r}$ & $\mathrm{r}$ & $\mathrm{r}$ & $\mathrm{r}$ & $\mathrm{r}$ & $\mathrm{r}$ \\
\hline Incisur shallow or deep & *Shallow & *Deep & *Shallow & $*$ Deep & *Deep & *Deep & *Deep \\
\hline No. of medial br. on rostrum (infold) & 8 & $6-7$ & $5-6 ?$ & $8 ?$ & 13 & $7-9$ & $7-8$ \\
\hline \multicolumn{8}{|l|}{ 1st antenna } \\
\hline no. of br. 2nd seg., d-la & $1-1$ & $1-1$ & $1-1$ & $1-1$ & $1-1$ & $1-1$ & $1-1$ \\
\hline no. of br. 3rd seg., d.-v & $2-1$ & $2-1$ & $2-1$ & $2-1$ & $2-1$ & $2-1$ & $2-1$ \\
\hline no. of br. 4 th seg., d.-v & $1-2$ & $1-2$ & $1-2$ & $1-2$ & $1-2$ & $1-2$ & $1-2$ \\
\hline no. of fil. on sens. br. & 0 & 1 & 2 & 3 & 2 & 1 & 1 \\
\hline no. of fil. on b.-br & 0 & 0 & 0 & 0 & 0 & 0 & 0 \\
\hline no. of fil. on c.-br. & 0 & 0 & 1 & 1 & 0 & 1 & 0 \\
\hline no. of fil. on g.-br. & 1 & 1 & 2 & 2 & 1 & 1 & 1 \\
\hline \multicolumn{8}{|l|}{ 2nd antenna } \\
\hline endop. no. of br. & 4 & $3-4$ & 1 or 4 & 5 & 4 & 4 & 4 \\
\hline \multicolumn{8}{|l|}{ Mandible } \\
\hline coxale + or - bifurcate endite & + & + & + & + & + & + & + \\
\hline basale, no. of d. br. mid.-dist. & $?$ & 3 & 3 & 3 & 3 & 4 & 3 \\
\hline basale, no. of thin v. br. & $?$ & 5 & $* 5$ & $* 3$ & $4 ?$ & *2 & 4 \\
\hline basale, no. of stout v. br. & $?$ & 3 & $* 2$ & $* 4$ & $2 ?$ & $* 4$ & 2 \\
\hline basale, total no. of v. br. & $?$ & 8 & 7 & 7 & 6 & 6 & 6 \\
\hline 1st end. seg., no. v. br. & $?$ & 2 & 2 & 2 & 2 & 2 & 2 \\
\hline 2nd end. seg., no. of d. br. & $?$ & 3 & 4 & $3-4$ & 3 & 3 & 3 \\
\hline 2nd end. seg., no. of v. br. excl. cl. & $?$ & 4 & 4 & 4 & 4 & 2 & 3 \\
\hline 3rd end. seg., no. of br. excl. cl. & $?$ & 4 & 3 & 3 & 3 & 6 & 4 \\
\hline Maxilla, no. br. on the 3 endites & 9 & $3-3-4$ & $?$ & $?$ & $6-5-6$ & $3-3-3$ & $2-3-3$ \\
\hline \multicolumn{8}{|l|}{ Fifth limb } \\
\hline no. br. on the 3 endites & $?$ & $3-3-5$ & $3-4-6$ & $?$ & $4-4-7$ & $(2-3)-5-8$ & $3-7-4$ \\
\hline ex. seg., no. primary teeth & $?$ & 4 & 4 & $* 4$ & 4 & 4 & 4 \\
\hline 1st ex. seg., no. teeth & $?$ & 3 & 3 & $* 3$ & 3 & 3 & 3 \\
\hline 2nd ex. seg., + or - bi-or trifurcate teeth & $?$ & + & + & + & + & + & + \\
\hline 3rd ex. seg., no. br. on each lobe & $2-2$ & $3-2$ & $3-2$ & $3-2$ & $3-2$ & $2-3$ & $2-2$ \\
\hline 4 th +5 th segs. no. br. & 4 & 5 & 4 & 4 & $3-4$ & $4-5$ & 4 \\
\hline \multicolumn{8}{|l|}{ Sixth limb } \\
\hline no. epipodial br. & $?$ & 2 & 2 & 2 & 2 & $1-2$ & 2 \\
\hline no. br. on the 4 endites & $?$ & $3-2-2-(2-3)$ & $3-2-2-2$ & $3-2-3-2$ & $(2-3)-2-3-3$ & $3-2-2-(2-3)$ & $2-2-3-2$ \\
\hline end-seg., no. br. & $?$ & $7-8$ & 6 & 6 & 7 & $7-8$ & 7 \\
\hline \multicolumn{8}{|l|}{ 7th limb } \\
\hline no. terminal cleaning br. & $3-3$ & $3-3$ & $3-3$ & $3-3$ & $3-3$ & $3-3$ & $3-3$ \\
\hline no. prox. cleaning br. & $2-2$ & $2-(1-2)$ & $(1-2)-(1-2)$ & $2-2$ & $2-2$ & $2-2$ & $2-2$ \\
\hline no. comb-teeth & $1-2$ & $(2-3)-(2-3)$ & $(3-4)-(3-4)$ & $3-4$ & $5-2$ & $* ? 4-2$ & $1-5$ \\
\hline Furca, no. of main claws & 4 & 4 & $* 4 ?$ & 3 & 3 & 4 & 4 \\
\hline Furca, no. of secondary claws & 2 & 2 & $* 3 ?$ & 3 & 3 & 2 & 2 \\
\hline Lateral Eyes, + or - & $?$ & + & + & + & + & + & + \\
\hline Specimen Number, \# = USNM & \#122907 & \#194087 & \#194254 & (1) & ZMH 27297 & \#154185 & \#158219 \\
\hline
\end{tabular}


(c)

\begin{tabular}{|c|c|c|c|c|c|c|c|}
\hline Species & irrostrata & judayi & kalkei & leloeuffi & licina & lomae & mollita \\
\hline Shell length, mm & 1.5 & 1.01 & 1.08 & 1.99 & 1.02 & 1.46 & 1.46 \\
\hline Surface with ribs (r) or smooth (s) & $\mathrm{r}$ & $\mathrm{r}$ & $\mathrm{r}$ & $\mathrm{r}$ & $\mathrm{r}$ & $\mathrm{r}$ & $\mathrm{r}$ \\
\hline Incisur shallow or deep & Deep & *Deep & *Deep & *Deep & *Deep & *Deep & *Shallow \\
\hline No. of medial br. on rostrum (infold) & $?$ & 7 & $7-8$ & 4 & $6-7$ & $10-12$ & $8-12$ \\
\hline \multicolumn{8}{|l|}{ 1st antenna } \\
\hline no. of br. 2nd seg., d-la & $1-1$ & $1-1$ & $1-1$ & $1-1$ & $1-1$ & $1-1$ & $1-1$ \\
\hline no. of br. 3rd seg., d.-v & $1-1$ & $2-1$ & $2-1$ & $1-1$ & $2-1$ & $2-1$ & $2-1$ \\
\hline no. of br. 4th seg., d.-v & $1-2$ & $1-2$ & $1-2$ & $1-2$ & $1-2$ & $1-2$ & $1-2$ \\
\hline no. of fil. on sens. br. & 0 & 1 & 1 & 0 & 0 & 3 & $3-4$ \\
\hline no. of fil. on b.-br & 0 & 0 & 0 & 0 & 0 & 0 & 0 \\
\hline no. of fil. on c.-br. & 0 & 0 & 1 & 0 & 1 & $0-1$ & $0-1$ \\
\hline no. of fil. on g.-br. & 0 & 1 & 1 & 0 & 1 & 0 & $2-3$ \\
\hline \multicolumn{8}{|l|}{ 2nd antenna } \\
\hline endop. no. of br. & 4 & 4 & 4 & 4 & 4 & 5 & 4 \\
\hline \multicolumn{8}{|l|}{ Mandible } \\
\hline coxale + or - bifurcate endite & 2 & $(2-5)$ & $(2-5)$ & $(2-4)$ & $(2-5)$ & $(2-5)$ & $(2-5)$ \\
\hline basale, no. of d. br. mid.-dist. & + & + & + & + & + & + & + \\
\hline basale, no. of thin v. br. & 3 & 3 & 3 & 2 & 3 & 3 & 3 \\
\hline basale, no. of stout v. br. & 4 & $* 2$ & 3 & 2 & 2 & $* 2$ & $* 3$ \\
\hline basale, total no. of v. br. & 6 & 6 & 4 & 6 & 2 & 5 & 7 \\
\hline 1st end. seg., no. of v. br. & 3 & 2 & 2 & 3 & 2 & 2 & 2 \\
\hline 2nd end. seg., no. of d. br. & 2 & 3 & 3 & 3 & 3 & 3 & 4 \\
\hline 2nd end. seg., no. of v. br. excl. cl. & 1 & 2 & 2 & 1 & 3 & 2 & 3 \\
\hline 3rd end. seg., no. of br. excl. cl. & 5 & $6 ?$ & 6 & 5 & 6 & 6 & 6 \\
\hline Maxilla, no. of br. on the 3 endites & $(5-6)-(5-6)-7$ & $?$ & $(2-3)-(2-3)-(2-3)$ & $(4-7)-4-5$ & $6-5-8$ & $5-4-(6-7)$ & $5-5-5$ \\
\hline \multicolumn{8}{|l|}{ 5th limb } \\
\hline no. of br. on the 3 endites & $2-3-4$ & $?$ & $3-6-6$ & $2-3-4$ & $3-5-6$ & $2-6-6$ & $3-5-8$ \\
\hline ex. seg., no. of primary teeth & $* 2$ & 4 & $* 4$ & 4 & $* 4$ & 4 & 4 \\
\hline 2nd ex. seg., no. of teeth & $* 3$ & 3 & $* 3$ & 3 & $* 3$ & 3 & 3 \\
\hline 2nd ex. seg., + or - bi-or trifurcate teeth & + & + & + & + & + & + & + \\
\hline 3rd ex. seg., no. of br. on each lobe & $2-2$ & $3-2$ & $2-3$ & $3-2$ & $3-(1$ or 2$)$ & $3-2$ & $3-2$ \\
\hline 4 th +5 th segs. no. of br. & 4 & 4 & $3-4$ & 4 & 4 & 5 & 4 \\
\hline \multicolumn{8}{|l|}{ 6th limb } \\
\hline no. of epipodial br. & 2 & 2 & 2 & 2 & 2 & 2 & 2 \\
\hline no. of br. on the 4 endites & $1-2-2-3$ & $2-2-2-2$ & $(2-3)-2-2-3$ & $3-2-2-2$ & $3-1-(2-3)-(2-3)$ & $2-1-2-2$ & $3-2-4-3$ \\
\hline end-seg., no. of br. & 7 & 7 & $7-8$ & 7 & 7 & 7 & 7 \\
\hline \multicolumn{8}{|l|}{ 7th limb } \\
\hline no. of terminal cleaning br. & 7 & $3-3$ & $2-3$ or $3-3$ & $2-3$ & $2-3$ or $3-3$ & $3-3$ & $3-3$ \\
\hline no. of prox. cleaning br. & $3-2$ & $2-2$ & $1-1$ & $2-2$ & $2-2$ & $2-2$ & $2-2$ \\
\hline no. of comb-teeth & $2-2$ & $3-2$ & $?$ & $! 8$ & $(2-3)-(2-3)$ & $5-3$ & $11-7$ \\
\hline Furca, no. of main claws & $* 3-3$ & 4 & 4 & 3 & 3 & 4 & 3 \\
\hline Furca, no. of secondary claws & 3 & 2 & 2 & 3 & 3 & 2 & 3 \\
\hline Lateral Eyes, + or - & - & + & + & $?$ & + & - & + \\
\hline Specimen Number, \# = USNM & \#152826 & \#158221 & \#159079 & \#149330 & \#152851 & $\# 158258$ & \#158001 \\
\hline
\end{tabular}


(d)

\begin{tabular}{|c|c|c|c|c|c|c|c|}
\hline Species & normani & ovata & pax & rex & rostrata & rotunda & schroederi \\
\hline Shell length, mm & 1.2 & 1.52 & 1 & 0.92 & 1.32 & 1.33 & 1.73 \\
\hline Surface with ribs (r) or smooth (s) & $\mathrm{r}$ & $\mathrm{r}$ & $\mathrm{r}$ & $\mathrm{r}$ & $\mathrm{r}$ & $\mathrm{r}$ & $\mathrm{r}$ \\
\hline Incisur shallow or deep & *Deep & $*$ Deep & *Deep & *Shallow & $*$ Deep & *Shallow & $*$ Deep \\
\hline No. of medial br. on rostrum (infold) & 5 & $4 ?$ & 7 & $?$ & $7-9$ & 7 & 17 \\
\hline \multicolumn{8}{|l|}{ 1st antenna } \\
\hline no. of br. 2nd seg., d-la & $1-1$ & $1-1$ & $1-1$ & $1-1$ & $1-1$ & $1-1$ & $1-0$ \\
\hline no. of br. 3rd seg., d.-v & $2-1$ & $2-1$ & $2-1$ & $2-1$ & $2-1$ & $2-1$ & $2-1$ \\
\hline no. of br. 4 th seg., d.-v & $1-2$ & $1-2$ & $1-2$ & $1-2$ & $1-2$ & $1-2$ & $1-2$ \\
\hline no. of fil. on sens. br. & 0 & 2 & 1 & 3 & 1 & 3 & 1 \\
\hline no. of fil. on b.-br & 0 & 0 & 0 & 0 & 0 & 0 & 0 \\
\hline no. of fil. on c.-br. & 0 & $0 ?$ & 1 & 1 & 1 & 1 & 0 \\
\hline no. of fil. on g.-br. & 1 & 1 & 1 & 2 & 1 & 2 & 2 \\
\hline \multicolumn{8}{|l|}{ 2nd antenna } \\
\hline endop. no. of br. 1st seg & 4 & 4 & 4 & 4 & 4 & 4 & 5 \\
\hline endop. no. of br. 2nd seg & abs. & abs. & abs. & abs. & abs. & abs. & abs. \\
\hline endop. no. of br. 3rd seg & abs. & abs. & abs. & abs. & abs. & abs. & abs. \\
\hline endop. no. of br. w/o setules & $(2-5)$ & $(2-5)$ & $(2-5)$ & $(2-5)$ & $(2-5)$ & $(2-5)$ & $(2-5)$ \\
\hline \multicolumn{8}{|l|}{ Mandible } \\
\hline coxale + or - bifurcate endite & + & + & + & + & + & + & + \\
\hline basale, no. of d. br. mid.-dist. & 3 & 3 & 3 & 3 & 3 & 3 & 3 \\
\hline basale, no. of thin v. br. & 4 & $* 5$ & $* 5$ & $* 3$ & $* 5$ & $* 5$ & $* 5$ \\
\hline basale, no. of stout v. br. & 2 & $* 2$ & $* 2$ & $* 4$ & $* 2$ & $* 2$ & $* 3$ \\
\hline basale, total no. of v. br. & 6 & 7 & 7 & 7 & 7 & 7 & $7-8$ \\
\hline 1st end. seg., no. of v. br. & 2 & 2 & 2 & 2 & 2 & 2 & 3 \\
\hline 2nd end. seg., no. of d. br. & 4 & 3 & 3 & 4 & 3 & 4 & 5 \\
\hline 2nd end. seg., no. of v. br. excl. cl. & 2 & 2 & 2 & 4 & 2 & 2 & $\begin{array}{l}2 \text { or } 3 \\
\text { (otd) }\end{array}$ \\
\hline 3rd end. seg., no. of br. excl. cl. & 6 & 3 & 6 & 3 & 6 & 6 & 4 \\
\hline \multicolumn{8}{|l|}{ Maxilla } \\
\hline no. of br. on the 3 endites & $6-5-5$ & $6-5-7$ & $\begin{array}{l}(4-6)-(4- \\
6)-(4-6)\end{array}$ & $\begin{array}{l}(2-3)-(2- \\
3)-(2-3)\end{array}$ & $\begin{array}{l}(4-6)-(4- \\
6)-(4-6)\end{array}$ & $6-4-5$ & $* 6-3-6$ \\
\hline \multicolumn{8}{|l|}{5 th $\operatorname{limb}$} \\
\hline no. of br. on the 3 endites & $3-5-7$ & $3-4-6$ & $2-4-6$ & $3-3-9$ & $2-4-6$ & $3-(4-5)-6$ & $\begin{array}{l}(1-3)-5- \\
(5-6)\end{array}$ \\
\hline ex. seg., no. of primary teeth & 5 & 4 & 4 & $* 4$ & 4 & 4 & 4 \\
\hline 2nd ex. seg., no. of teeth & 3 & 3 & 3 & $* 3$ & 3 & 3 & 3 \\
\hline $\begin{array}{l}\text { 2nd ex. seg., + or - bi-or trifurcate } \\
\text { teeth }\end{array}$ & + & + & + & + & + & - & + \\
\hline 3rd ex. seg., no. of br. on each lobe & $3-2$ & $3-2$ & $1-2$ & $3-2$ & $2-2$ & $4-2$ & $3-2$ \\
\hline 4 th +5 th segs. no. of br. & 4 & 4 & 4 & $3-4$ & 4 & 4 & 4 \\
\hline \multicolumn{8}{|l|}{ 6th limb } \\
\hline no. of epipodial br. & 2 & 2 & 2 & 2 & 1 & 2 & 2 \\
\hline no. of br. on the 4 endites & $3-2-2-3$ & $\begin{array}{l}3-1-(2- \\
3)-3\end{array}$ & $3-1-2-2$ & $3-2-2-2$ & $3-1-2-3$ & $3-4-3-3$ & $3-3-3-3$ \\
\hline end-seg., no. of br. & 7 & 7 & 7 & 6 & 7 & 6 & 6 \\
\hline \multicolumn{8}{|l|}{ 7th limb } \\
\hline no. of terminal cleaning br. & $3-3$ & $3-3$ & $2-3$ or $3-3$ & $\begin{array}{l}(2-3)-(2- \\
3)\end{array}$ & $3-3$ & $3-3$ & $3-3$ \\
\hline no. of prox. cleaning br. & $?$ & $2-2$ & $1-2$ or $2-2$ & $\begin{array}{l}(1-2)-(1- \\
2)\end{array}$ & $2-2$ & $3-3$ & $2-2$ \\
\hline no. of comb-teeth & $2-5$ & $13-9$ & $11-6$ & $6-4$ & $11-6$ & $8-8$ & $5-2$ \\
\hline Furca, no. of main claws & 3 & 3 & 4 & 3 & 4 & 3 & 4 \\
\hline Furca, no. of secondary claws & 3 & 3 & 2 & 3 & 2 & 3 & 2 \\
\hline Lateral Eyes, + or - & + & + & - & + & - & + & + \\
\hline Specimen Number, \# = USNM & $\# 193675$ & $\# 137686$ & $\begin{array}{c}\mathrm{ZMH} \\
27314-3\end{array}$ & \#193415 & \#158222 & \#158214 & \#194472 \\
\hline
\end{tabular}


(e)

\begin{tabular}{|c|c|c|c|}
\hline Species & sterreri & $\operatorname{tryx}$ & vox \\
\hline Shell length, mm & 1.1 & 1.1 & 0.91 \\
\hline Surface with ribs (r) or smooth (s) & $\mathrm{r}$ & $\mathrm{r}$ & $\mathrm{r}$ \\
\hline Incisur shallow or deep & $*$ Deep & *Shallow & *Shallow \\
\hline No. of medial br. on rostrum (infold) & $6-8$ & 6 & 8 \\
\hline \multicolumn{4}{|l|}{ 1st antenna } \\
\hline no. of br. 2nd seg., d-la & $1-1$ & $1-0$ & $1-1$ \\
\hline no. of br. 3rd seg., d.-v & $(1-2)-1$ & $2-1$ & $2-1$ \\
\hline no. of br. 4 th seg., d.-v & $1-2$ & $1-2$ & $1-2$ \\
\hline no. of fil. on sens. br. & 1 & 2 & 2 \\
\hline no. of fil. on b.-br & 0 & 0 & 0 \\
\hline no. of fil. on c.-br. & 1 & 2 & 1 \\
\hline no. of fil. on g.-br. & 1 & 2 & 1 \\
\hline \multicolumn{4}{|l|}{ 2nd antenna } \\
\hline endop. no. of br. & 4 & 5 & $3-4$ \\
\hline \multicolumn{4}{|l|}{ Mandible } \\
\hline coxale + or - bifurcate endite & + & + & + \\
\hline basale, no. of d. br. mid.-dist. & 3 & 3 & 3 \\
\hline basale, no. of thin v. br. & $* 5$ & 3 & 4 \\
\hline basale, no. of stout v. br. & $* 2$ & 2 & 3 \\
\hline basale, total no. of v. br. & 7 & 5 & 7 \\
\hline 1st end. seg., no. of v. br. & 2 & 2 & 2 \\
\hline 2nd end. seg., no. of d. br. & 3 & 3 & 3 \\
\hline 2nd end. seg., no. of v. br. excl. cl. & 2 & 4 & 4 \\
\hline 3rd end. seg., no. of br. excl. cl. & 6 & 4 & 3 \\
\hline Maxilla, no. of br. on the 3 endites & $5-5-6$ & $3-2-3$ & $2-4-3$ \\
\hline \multicolumn{4}{|l|}{5 th limb } \\
\hline no. of br. on the 3 endites & $3-5-6$ & $4-4-6$ & $3-(4-6)-4$ \\
\hline ex. seg., no. of primary teeth & 4 & 4 & 4 \\
\hline 2nd ex. seg., no. of teeth & 3 & 3 & 3 \\
\hline 2nd ex. seg., + or - bi-or trifurcate teeth & + & + & + \\
\hline 3rd ex. seg., no. of br. on each lobe & $3-2$ & $3-2$ & $3-2$ \\
\hline 4 th +5 th segs. no. of br. & 4 & 4 & 4 \\
\hline \multicolumn{4}{|l|}{ 6th limb } \\
\hline no. of epipodial br. & 2 & 2 & 1 \\
\hline no. of br. on the 4 endites & $(2-3)-2-3-3$ & $3-2-3-3$ & $3-2-2-2$ \\
\hline end-seg., no. of br. & 7 & 6 & 7 \\
\hline 7th limb & $3-3$ & $3-3$ & $3-3$ \\
\hline no. of terminal cleaning br. & $2-2$ & $2-2$ & $2-2$ \\
\hline no. of prox. cleaning br. & $5-3$ & $5-3$ & $?$ \\
\hline \multicolumn{4}{|l|}{ no. of comb-teeth } \\
\hline Furca, no. of main claws & 4 & 3 & 4 \\
\hline Furca, no. of secondary claws & 2 & 3 & 2 \\
\hline Lateral Eyes, + or - & + & + & - \\
\hline Specimen Number, \# = USNM & \#158115 & \#194147 & \#158309 \\
\hline (1) after Poulsen, [10] & & & \\
\hline
\end{tabular}

abs.: absent; br.: bristles; cl.: claw; d: dorsal; dist.: distal; endop.: endopodite; ex.: exopod; fil.: filaments; no.: number; prox.: proximal; seg.: segment; sens: sensory; v: ventral.; ?: unknown; *: after Poulsen [10]. (Only males have been described for the species fusca, mortenseni, sagax, and tridens; see Table 4). 
TABLE 8: Summary of characteristics of mature males in Rutiderma.

(a)

\begin{tabular}{|c|c|c|c|c|c|c|c|}
\hline Species & apex & $\operatorname{arx}$ & darbyi & $d u x$ & flex & fusca & gerdhartmanni \\
\hline Shell length, mm & 1.29 & 1.3 & 1.12 & 1.05 & 1.21 & 1.1 & 1.48 \\
\hline Surface with ribs (r) or smooth (s) & $\mathrm{r}$ & s & $\mathrm{r}$ & $\mathrm{r}$ & $\mathrm{r}$ & $\mathrm{r}$ & $\mathrm{r}$ \\
\hline Incisur shallow or deep & Shallow! & Deep & Deep & *Shallow & ${ }^{*}$ Deep & Deep & ${ }^{*}$ Deep \\
\hline No. of medial br. on rostrum (infold) & $?$ & 6 & 8 & 8 & 7 & 5 & $(12-16)$ \\
\hline \multicolumn{8}{|l|}{ 1st antenna } \\
\hline no. of br. 2nd seg., d-la & $1-1$ & $1-1$ & $1-1$ & $1-1$ & $1-1$ & $1-1$ & $1-1$ \\
\hline no. of br. 3rd seg., d.-v & $2-1$ & $2-1$ & $2-1$ & $2-1$ & $2-1$ & $2-1$ & $2-1$ \\
\hline no. of br. 4th seg., d.-v & $1-3$ & $1-3$ & $1-3$ & $1-3$ & $1-3$ & $1-3$ & $1-3$ \\
\hline no. of fil. on sens. br. & Numerous! & Numerous & Numerous & Numerous & Numerous & 10 & 20 \\
\hline no. of fil. on b.-br & 2 & 2 & 2 & 2 & 2 & 2 & 2 \\
\hline no. of fil. on c.-br. & 11 & 11 & 10 & Numerous & 10 & 20 & 9 \\
\hline no. of fil. on g.-br. & $(1-2)$ & 2 & 1 & 1 & $(1-2)$ & 2 & 1 \\
\hline \multicolumn{8}{|l|}{ 2nd antenna } \\
\hline endop. no. of br. on 1st seg. & 5 & 5 & 5 & 5 & 5 & 5 & 5 \\
\hline endop. no. of br. on 2 nd seg. & 2 & 2 & 2 & 2 & 2 & 2 & 2 \\
\hline endop. no. of br. on 3rd seg. & 3 & 3 & 3 & 3 & 3 & 3 & 3 \\
\hline \multicolumn{8}{|l|}{ Mandible } \\
\hline basale, no. of d. br. mid.-dist. & 3 & 3 & 3 & 3 & 3 & 3 & 3 \\
\hline basale, no. of thin v. br. & $* 5$ & $* 5$ & $* 5$ & $* 4$ & $* 4$ & 5 & $* 6$ \\
\hline basale, no. of stout v. br. & $* 1$ & $* 0$ & $* 0$ & ${ }^{*} 0$ & $* 0$ & 0 & $* 0$ \\
\hline basale, total no. of v. br. & 6 & 5 & 5 & 4 & 4 & 5 & 6 \\
\hline 1st end. seg., no. of v. br. & 2 & 2 & 2 & 2 & 2 & 2 & 2 \\
\hline 2nd end. seg., no. of d. br. & 3 & 4 & 3 & 3 & 3 & 4 & 3 \\
\hline 2nd end. seg., no. of v. excl. cl. & 6 & 5 & 6 & 6 & 6 & 6 & 6 \\
\hline 3rd end. seg, no. of br. excl. cl. & $(4-5)$ & 4 & 5 & 4 & 5 & 5 & 5 \\
\hline Maxilla, no. br. on the 3 endites & $5-4-6$ & $?$ & $4-?-?$ & $?$ & 11 & $5-4-4$ & $6-?-?$ \\
\hline \multicolumn{8}{|l|}{ Fifth Limb } \\
\hline no. br. on the 3 endites & $(3-4)-4-6$ & $2-2-3$ & $3-4-6$ & $2-4-3$ & $?$ & $3-4-5$ & $3-(3-5-6-7)$ \\
\hline 3rd exop. seg., no. br. each ?lobe & $3-2$ & $3-2$ & $3-2$ & $?$ & $?$ & $1-2$ & $3-2$ \\
\hline 4 th +5 th segs. no. of br. & 4 & 4 & 4 & $?$ & $?$ & 5 & 4 \\
\hline \multicolumn{8}{|l|}{ Sixth Limb } \\
\hline no. epipodial br. & 1 & 2 & 2 & 2 & 2 & 1 & $(1-2)$ \\
\hline no. of br. on the 4 endites & $3-2-2-2$ & $2-2-3-3$ & $?-?-2-3$ & $3-2-2-3$ & $2-2-3-3$ & $2-2-2-2$ & $3-(2-3)-2-3$ \\
\hline end-seg., no. of br. & 7 & 6 & 7 & 7 & 7 & 6 & 7 \\
\hline \multicolumn{8}{|l|}{ 7th limb } \\
\hline no. of terminal cleaning br. & $2-2$ & $2-2$ & $2-2$ & $2-2$ & $2-2$ & $2-2$ & $2-2$ \\
\hline no. of prox. cleaning br. & $1-2$ & $2-2$ & $2-2$ & $2-2$ & $2-2$ & $2-2$ & $?$ \\
\hline no. of comb-teeth & $3-3$ & $(2-3)(2-3)$ & $3-2$ & $2-2$ & $2-2$ & $2-2$ & $3-2$ \\
\hline Furca, no. of main claws & 4 & 3 & 4 & 4 & 4 & 3 & 3 \\
\hline Furca, no. of secondary claws & 2 & 3 & 2 & 2 & 2 & 3 & 3 \\
\hline Copulatory limbs long or short & sh. & $*$ sh. & $*$ sh. & $*$ sh. & $* 1$ & sh. & $?$ \\
\hline Specimen Number, \# = USNM & \#158264 & \#193409 & $\# 152851$ & \#194091 & \#1021462 & ZMUC & ZMH 27297 \\
\hline
\end{tabular}


(b)

\begin{tabular}{|c|c|c|c|c|c|c|c|}
\hline Species & gyre & hartmanni & judayi & kalkei & licina & lomae & mollita \\
\hline Shell length, mm & 1.12 & 1.06 & 1.03 & 1.14 & 1.08 & 1.21 & 1.47 \\
\hline Surface with ribs (r) or smooth (s) & $\mathrm{r}$ & $\mathrm{r}$ & $\mathrm{r}$ & $\mathrm{r}$ & $\mathrm{r}$ & $\mathrm{r}$ & $\mathrm{r}$ \\
\hline Incisur shallow or deep & $*$ Deep & ${ }^{*}$ Deep & *Shallow & ${ }^{*}$ Deep & $*$ Deep & ${ }^{*}$ Deep & ${ }^{*}$ Deep \\
\hline No. medial br. on rostrum (rostral infold) & $(6-8)$ & 8 & $(5-6)$ & $(7-8)$ & 6 & 8 & 11 \\
\hline \multicolumn{8}{|l|}{ 1st antenna } \\
\hline no. of br. 2nd seg., d-la & $1-1$ & $1-1$ & $1-1$ & $1-1$ & $1-1$ & $1-1$ & $1-1$ \\
\hline no. of br. 3rd seg., d.-v & $2-1$ & $2-1$ & $2-1$ & $2-1$ & $2-1$ & $2-1$ & $2-1$ \\
\hline no. of br. 4 th seg., d.-v & $1-3$ & $1-3$ & $1-3$ & $1-3$ & $1-3$ & $1-3$ & $1-3$ \\
\hline no. of fil. on sens. br. & Numerous & 25 & Numerous & Numerous & Numerous & Numerous & Numerous \\
\hline no. of fil. on b.-br & 3 & 2 & 2 & 3 & 2 & 2 & 2 \\
\hline no. of fil. on c.-br. & 12 & 14 & 10 & 11 & 11 & 11 & 11 \\
\hline no. of fil. on g.-br. & 2 & 1 & 1 & 1 & 1 & 1 & 2 \\
\hline \multicolumn{8}{|l|}{ 2nd antenna } \\
\hline endop. no. of br. on 1st seg. & 5 & 4 & 5 & 5 & 5 & 5 & 5 \\
\hline endop. no. of br. on 2 nd seg. & 2 & 2 & 2 & 2 & 2 & 2 & 2 \\
\hline endop. no. of br. on 3rd seg. & 3 & 3 & 3 & 3 & 1 & 1 & 3 \\
\hline \multicolumn{8}{|l|}{ Mandible } \\
\hline basale, no. of d. br. mid.-dist. & 3 & 3 & 3 & 3 & 3 & 3 & 3 \\
\hline basale, no. of thin v. br. & $* 3$ & $* 5$ & $* 3$ & $?$ & $* 4$ & $* 4$ & $* 6$ \\
\hline basale, no. of stout v. br. & $* 3$ & $* 1$ & $* 3$ & $?$ & $* 2$ & $* 2$ & $* 0$ \\
\hline basale, total no. of v. br. & 6 & 6 & 6 & 5 & 6 & 6 & 6 \\
\hline 1st end. seg., no. of v. br. & 2 & 2 & 2 & 2 & 2 & 2 & 2 \\
\hline 2nd end. seg., no. of v. br. excl. cl. & 6 & 6 & 6 & 6 & 5 & 6 & 6 \\
\hline 3rd end. seg., no. of br. excl. cl. & 5 & 6 & 5 & 5 & 5 & 5 & 4 \\
\hline Maxilla, no. of br. on the 3 endites & $?$ & $?$ & $?$ & $?$ & ? & $5-5-7$ & $?$ \\
\hline \multicolumn{8}{|l|}{5 th limb } \\
\hline no. of br. on the 3 endites & $3-4-7$ & $2-3-5$ & $3-4-7$ & $2-4-(7-8)$ & $2-3-5$ & $3-4-8$ & $4-5-7$ \\
\hline 3rd exop. seg., no. of br. on each lobe & $2-2$ & $3-2$ & $2-2$ & $(1-2)-2$ & $3-2$ & $3-2$ & $2-2$ \\
\hline 4 th +5 th segs. no. of br. & 4 & 4 & 4 & 4 & 3 & 4 & 4 \\
\hline \multicolumn{8}{|l|}{ 6th limb } \\
\hline no. of epipodial br. & 2 & 2 & 2 & 2 & 2 & 2 & 2 \\
\hline no. of br. on the 4 endites & $3-2-2-3$ & $3-2-3-3$ & $3-2-2-3$ & $3-2-2-?$ & $3-1-2-2$ & $3-2-1-2$ & $3-2-4-3$ \\
\hline end-seg., no. of br. & 7 & 7 & 7 & 7 & $(7-8)$ & 6 & 7 \\
\hline \multicolumn{8}{|l|}{7 th $\operatorname{limb}$} \\
\hline no. of terminal cleaning br. & $2-2$ & $2-2$ & $2-2$ & $2-2$ & $2-2$ & $2-2$ & $2-2$ \\
\hline no. of prox. cleaning br. & $2-2$ & $?$ & $2-2$ & $1-1$ & $2-2$ & $2-2$ & $2-2$ \\
\hline no. of comb-teeth & $(2-3)-(3-4)$ & $2-2$ & $(2-3)-(2-3)$ & $(2-3)-(2-3)$ & $(2-3)-(2-3)$ & $3-3$ & $2-3$ \\
\hline Furca, no. of main claws & 4 & 4 & 4 & 4 & 3 & 4 & 3 \\
\hline Furca, no. of secondary claws & 2 & 2 & 2 & 2 & 3 & 2 & 3 \\
\hline Copulatory limbs long or short & 1. & 1. & $?$ & $*$ sh. & $* 1$ & 1. & $?$ \\
\hline Specimen Number, \# = USNM & \#157993 & \#158218 & \#158220 & \#159069B & \#157878 & \#158260 & \#158209 \\
\hline
\end{tabular}

Only female or juvenile: arcuatilis, arx, ferax, chessi, cohenae, compressa, dinochelata, exrex, ferax, irrostrata, leloeuffi, rex, schroederi, tridens, tryx. 
(c)

\begin{tabular}{|c|c|c|c|c|c|c|c|c|}
\hline Species & mortenseni & normani & i ovata & rostrata & rotunda & $\operatorname{sagax}$ & sterreri & vox \\
\hline Shell length, mm & 1.07 & 1.2 & 1.59 & 1.29 & 1.24 & 1.05 & 0.95 & 0.96 \\
\hline Surface with ribs (r) or smooth (s) & $\mathrm{r}$ & $\mathrm{r}$ & $\mathrm{r}$ & s & $s !$ & $\mathrm{r}$ & $\mathrm{r}$ & $\mathrm{r}$ \\
\hline Incisur shallow or deep & Deep & Deep & $*$ Deep & $*$ Deep & * Shallow & *Deep & *Shallow & *Deep? \\
\hline No. medial br. on rostrum (rostral infold) & 12 & 5 & 6 & $(7-9)$ & $(6-7)$ & $(4-5)$ & 8 & 8 \\
\hline \multicolumn{9}{|l|}{ 1st antenna } \\
\hline no. of br. 2nd seg., d-la & $1-1$ & $1-1$ & $1-1$ & $1-1$ & $1-1$ & $1-1$ & 1 & $1-1$ \\
\hline no. of br. 3rd seg., d.-v & $2-1$ & $2-1$ & $2-1$ & $2-1$ & $2-1$ & $2-1$ & $2-1$ & $2-1$ \\
\hline no. of br. 4 th seg., d.-v & $1-3$ & $1-3$ & $1-3$ & $1-3$ & $1-3$ & $1-3$ & $1-3$ & $1-3$ \\
\hline no. of fil. on sens. br. & 35 & 20 & 23 & Numerous & Numerous & Numerous & Numerous & Numerous \\
\hline no. of fil. on b.-br & 1 & 2 & 2 & 2 & 2 & 2 & 2 & 2 \\
\hline no. of fil. on c.-br. & 13 & 15 & 12 & 11 & 12 & Numerous & 9 & 10 \\
\hline no. of fil. on g.-br. & 1 & 1 & 1 & 1 & 2 & 2 & 1 & 1 \\
\hline \multicolumn{9}{|l|}{ 2nd antenna } \\
\hline endop. no. of br. on 1st seg. & 5 & 5 & 5 & 5 & 5 & 5 & 5 & 5 \\
\hline endop. no. of br. on 2 nd seg. & 2 & 2 & 2 & 2 & 2 & 2 & 2 & 2 \\
\hline endop. no. of br. on 3rd seg. & 3 & 3 & 3 & 2 & 3 & 3 & 3 & 3 \\
\hline exop., ser. no. of segs. w/o setules & 2 & 2 & 2 & 2 & 2 & 2 & 2 & 2 \\
\hline \multicolumn{9}{|l|}{ Mandible } \\
\hline basale, no. of d. br. mid.-dist. & 3 & 3 & 3 & 3 & 3 & 3 & 3 & 3 \\
\hline basale, no. of thin v. br. & 4 & 1 & $* 6$ & 4 & $* 6$ & $* 6$ & $* 5$ & 5 \\
\hline basale, no. of stout v. br. & 0 & 0 & $* 0$ & 2 & $* * 0$ & $* 0$ & $* 0$ & 0 \\
\hline basale, total no. of v. br. & 4 & 1 & 6 & 6 & 6 & 6 & 5 & 5 \\
\hline 1st end. seg., no. of v. br. & 2 & 2 & 2 & 2 & 2 & 2 & 2 & 2 \\
\hline 2nd end. seg., no. of d. br. & 3 & 3 & 3 & 3 & 4 & 4 & 3 & 3 \\
\hline 2nd end. seg., no. of v. br. excl. cl. & 6 & 6 & 6 & 6 & 6 & 5 & 6 & 6 \\
\hline 3rd end. seg., no. of br. excl. cl. & 5 & 6 & 5 & 5 & 4 & 4 & 4 & 4 \\
\hline Maxilla, no. of br. on the 3 endites & $5-4-4$ & $3-3-2$ & $5-5-6$ & $?$ & $?$ & $?$ & $?$ & $?$ \\
\hline \multicolumn{9}{|l|}{5 th limb } \\
\hline no. of br. 3 endites & $3-5-5$ & 3 & $2-4-5$ & $2-4-4$ & $3-4-6$ & $3-4-7$ & $3-4-7$ & $3-3-6$ \\
\hline 3rd exop. seg., no. br. on each lobe & $2-2$ & $?$ & $3-2$ & $3-2$ & $2($ otd $)-2$ & $3-2$ & $3-2$ & $3-2$ \\
\hline 4 th +5 th segs. no. br. & 5 & $?$ & 4 & 4 & 4 & 5 & 4 & 4 \\
\hline \multicolumn{9}{|l|}{ 6th limb } \\
\hline no. of epipodial br. & 1 & 1 & $(1-2)$ & 1 & 2 & 2 & 2 & 1 \\
\hline no. of br. on the 4 endites & $3-2-3-3$ & $2-2-2-3$ & $3-1-2-3$ & $3-1-3-3$ & $3-2-(2-3)-(2-3)$ & $3-2-2-3$ & $3-2-3-3$ & $3-2-2-2$ \\
\hline end-seg., no. of br. & 7 & 7 & 7 & 7 & 6 & 6 & 7 & 6 \\
\hline \multicolumn{9}{|l|}{7 th $\operatorname{limb}$} \\
\hline no. of terminal cleaning br. & $2-2$ & $2-2$ & $2-2$ & $2-2$ & $2-2$ & $2-2$ & $2-2$ & $2-2$ \\
\hline no. of prox. cleaning br. & $2-2$ & $2-2$ & $2-2$ & $2-2$ & $2-2$ & $2-2$ & $2-2$ & $2-2$ \\
\hline no. of comb-teeth & $2-2$ & $2-3$ & $13-9$ & $(2-3)-(2-3)$ & $3-2$ & $1-1$ & $(2-3)-(2-3)$ & $3-3$ \\
\hline Furca, no. of main claws & 4 & 3 & 3 & 4 & 3 & 3 & 4 & 4 \\
\hline Furca, no. of secondary claws & 2 & 3 & 3 & 2 & 3 & 4 & 2 & 2 \\
\hline Copulatory limbs long=l or short=sh. & sh. & 1. & 1. & sh. & $?$ & sh. & $* 1$ & $*$ sh. \\
\hline Specimen Number, \# = USNM & ZMUC7406 & \#193675 & \#137687 & \#158228 & \#158215 & AM P45365 & \#158573 & \#158316 \\
\hline
\end{tabular}

abs.: absent; br.: bristles; d: dorsal; dist.: distal; endop.: endopodite; ex.: exopod; fil.: filaments; no.: number; prox.: proximal; seg.: segment; sens: sensory; v: ventral. (Only female or juvenile: arcuatilis, arx, ferax, chessi, cohenae, compressa, dinochelata, exrex, ferax, irrostrata, leloeuffi, rex, schroederi, tridens, tryx.) *: Poulsen [10]. 
TABLE 9: Summary of characteristics of genera Metaschisma and Scleraner.

\begin{tabular}{|c|c|c|c|c|}
\hline \multirow{2}{*}{$\begin{array}{l}\text { Scientific Name } \\
\text { Sex }\end{array}$} & \multirow{2}{*}{$\frac{\text { Metaschisma nex }}{\text { Female }}$} & \multicolumn{2}{|c|}{ Scleraner trifax } & \multirow{2}{*}{$\frac{\text { Scleraner chacaoi }}{\text { Female }}$} \\
\hline & & Female & Male & \\
\hline Maturity & $\mathrm{m}$. & $\mathrm{m}$. & $\mathrm{m}$. & $\mathrm{m}$. \\
\hline Shell length, mm & 1.49 & 1.77 & 1.96 & 1.32 \\
\hline Surface with ribs (r) or smooth (s) & s & $\mathrm{r}$ & s & s \\
\hline Incisur shallow or deep & $*$ deep & $*$ deep & $*$ deep & $*$ deep \\
\hline $\begin{array}{l}\text { No. medial br. on rostrum } \\
\text { (rostral infold) }\end{array}$ & $(16-20)$ & 15 & 12 & 11 \\
\hline \multicolumn{5}{|l|}{ 1st antenna } \\
\hline no. br. 2nd seg., d-la-v. & $1-1-1$ & $1-1$ & $1-1$ & $1-1-1$ \\
\hline no. br. 3rd seg., d-v & $1-1$ & $1-2$ & $2-1$ & $1-2$ \\
\hline no. br. 4th seg., d-v & $1-3$ & $2-2$ & $2-3$ & $1-2$ \\
\hline no. fil. on sens. br. & 4 & 2 & Numerous & 2 \\
\hline no. fil. on b.-br & 1 & 0 & 3 & 0 \\
\hline no. fil. on c.-br. & 8 & 3 & 5 & 0 \\
\hline no. fil. on g.-br. & 2 & 2 & 2 & 2 \\
\hline \multicolumn{5}{|l|}{ 2nd antenna } \\
\hline endop. no. br. & 5 & 5 & 5 & 5 \\
\hline endop. no. br. 2nd seg. & 2 & 1 & 2 & 2 \\
\hline endop. no. br. 3rd seg. & abs. & abs. & 3 & abs. \\
\hline \multicolumn{5}{|l|}{ Mandible } \\
\hline coxale + or - furcate endite & + & + & - & + \\
\hline basale, no. d. br. mid.-dist. & 3 & 3 & 3 & 2 \\
\hline basale, no. thin v. br. & $* 5$ & $?$ & $* 9$ & *6 \\
\hline basale, no. stout v. br. & $* 2$ & $?$ & $* 0$ & $* 0$ \\
\hline basale, total no. br. & 7 & 11 & 9 & $(6-7)$ \\
\hline ex. absent, present or rud. & rud. & abs. & Present & abs. \\
\hline 1st end. seg., no. v. br. & 4 & 3 & 3 & 3 \\
\hline 2nd end. seg., no. d. br. & 6 & 7 & 6 & 7 \\
\hline 2nd end. seg., no. v. br. excl. claw & 2 & 5 & 6 & 2 \\
\hline 3rd end. seg., no. br. excl. cl. & 7 & 4 & 6 & 4 \\
\hline Maxilla, br. on endites & $6-5-3$ & $?$ & $9-6-5$ & $7-3-4$ \\
\hline \multicolumn{5}{|l|}{ 5th limb } \\
\hline br. on endites & $2-2-4$ & $3-5-7$ & $3-5-8$ & $3-4-5$ \\
\hline ex. seg., no. prim. teeth & 1 & 4 & abs. & 4 \\
\hline 2nd ex. seg., no. teeth & 3 & 3 & abs. & 2 \\
\hline 2nd ex. seg., + or - bi-or & & & & \\
\hline trifurcate teeth & + & + & - & + \\
\hline 3rd ex. seg., no. br. each lobe & $1-2$ & $3-2$ & $3-2$ & $3-2$ \\
\hline 4 th +5 th segs. no. br. & 4 & 4 & 5 & 8 \\
\hline \multicolumn{5}{|l|}{ 6th limb } \\
\hline no. epipodial br. & 2 & 2 & 2 & 2 \\
\hline no. br. on 4 endites & $3-(2-3)-(2-3)-2$ & $3-2-4-3$ & $3-2-4-3$ & $3-3-3-3$ \\
\hline end.-seg., no. br. & 10 & $(7-8)$ & $(7-8)$ & 8 \\
\hline \multicolumn{5}{|l|}{ 7th limb } \\
\hline no. terminal cleaning br. & $2-2$ & $3-3$ & $2-2$ & $3-3$ \\
\hline no. prox. cleaning br. & $2-2$ & $2-2$ & $1-1$ & $1-1$ \\
\hline no. comb-teeth & $17-0$ & $9-2$ & $7-2$ & $7-2$ \\
\hline \multicolumn{5}{|l|}{ Furca } \\
\hline no. main claws & 4 & 3 & 3 & 4 \\
\hline no. secondary claws & 7 & 3 & 3 & 5 \\
\hline Lateral eyes, + or - & + & + & + & - \\
\hline Copulatory limbs long or short & abs. & abs. & sh. & abs. \\
\hline Specimen No. $(\#=$ USNM) & \# 193930 & \# 193922 & \# 194019 & ZMH K 27302 \\
\hline
\end{tabular}

abs.: absent; br.: bristles; d: dorsal; dist.: distal; endop.: endopodite; ex.: exopod; fil.: filaments; 1: lateral; m.: mature; no.: number; prim.: primary; prox.: proximal; rud.: rudimentary; seg.: segment; sens: sensory; v: ventral, *: Poulsen [10]. 
TABLE 10: Summary of characteristics of males and females of Alternochelata, Rutiderma, and Scleraner.

\begin{tabular}{|c|c|c|c|c|c|c|}
\hline & \multicolumn{2}{|c|}{ Alternochelata } & \multicolumn{2}{|c|}{ Rutiderma } & \multicolumn{2}{|c|}{ Scleraner } \\
\hline & Male & Female & Male & Female & Male & Female \\
\hline Shell length, mm & $1.36-1.38$ & $1.18-1.37$ & $0.95-1.59$ & $0.91-1.99$ & 1.96 & $1.32-1.77$ \\
\hline Surface with ribs (r) or smooth (s) & s & s & s & $\mathrm{r} / \mathrm{s}$ & s & $\mathrm{r} / \mathrm{s}$ \\
\hline Incisur shallow or deep & Deep & Deep & Shallow/deep & Shallow/deep & Deep & Deep \\
\hline No. of medial br. on rostrum (infold) & 6,7 & $6-12$ & $4-9,11-16$ & $4-17$ & 12 & 11,15 \\
\hline \multicolumn{7}{|l|}{ 1st antenna } \\
\hline no. of br. 2nd seg., d-la & $1-1-1$ & $1-1-(0,1)$ & $1-1$ & $1-(0,1)$ & $1-1$ & $1-1$ \\
\hline no. of br. 3rd seg., d.-v & $1-1$ & $1-1$ & $2-1$ & $(1,2)-1$ & $2-1$ & $1-2$ \\
\hline no. of br. 4 th seg., d.-v & $1-4$ & $1-3$ & $1-3$ & $1-2$ & $2-3$ & $(1,2)-2$ \\
\hline no. of fil. on sens. br. & 33 , numerous & 1,3 & $\begin{array}{l}10,20,23,25 \\
25, \text { numerous }\end{array}$ & $0-5$ & Numerous & 2 \\
\hline no. of fil. on b.-br & 3 & 0,1 & $1-3$ & 0 & 3 & 0 \\
\hline no. of fil. on c.-br. & 12,13 & 1,3 & $\begin{array}{l}\text { 9-15, 20, } \\
\text { numerous }\end{array}$ & $0-2$ & 5 & 0,3 \\
\hline no. of fil. on g.-br. & $1-3$ & 1,3 & $1-2$ & $0-3$ & 2 & 2 \\
\hline \multicolumn{7}{|l|}{ 2nd antenna } \\
\hline endop. no. of br. on 1st seg. & 6 & 5,6 & 4,5 & $1,3-5$ & 5 & 5 \\
\hline endop. no. of br. on 2 nd seg. & 2 & 1,2 & 2 & & 2 & 1,2 \\
\hline endop. no. of br. on 3 rd seg. & 2,3 & 0 & $1-3$ & & 3 & 0 \\
\hline exop., ser. no. of segs. w/o setules & 2 & $2-5$ & 2 & & 2 & $2-5$ \\
\hline \multicolumn{7}{|l|}{ Mandible } \\
\hline basale, no. of d. br. mid.-dist. & 3 & 3,4 & 3 & $2-4$ & 3 & 2,3 \\
\hline basale, no. of thin v. br. & 5,6 & 3,5 & $1,3-6$ & $0-5$ & 9 & 6 \\
\hline basale, no. of stout v. br. & 0,3 & 2,3 & $0-3$ & $2-4$ & 0 & 0 \\
\hline basale, total no. of v. br. & 6,8 & 6,7 & $1,4-6$ & $2,4-8$ & 9 & $6,7,11$ \\
\hline 1st end. seg., no. of v. br. & 3 & 2,3 & 2 & 2,3 & 3 & 3 \\
\hline 2nd end. seg., no. of d. br. & 7 & $5-7$ & 3,4 & $2-5$ & 6 & 7 \\
\hline 2nd end. seg., no. of v. br. excl. cl. & 6 & 2,4 & 5,6 & $1-5$ & 6 & 2,5 \\
\hline 3rd end. seg., no. of br. excl. cl. & 5,6 & 3,5 & $4-6$ & $3-6$ & 6 & 4 \\
\hline Maxilla, no. of br. on the 3 endites & $\begin{array}{c}(5,6)-(4,6)- \\
(4,6,7)\end{array}$ & $\begin{array}{c}(3,7,8)- \\
(2,4,5)-(4-7)\end{array}$ & $\begin{array}{c}(3-6)-(3- \\
5)-(2,4,6,7)\end{array}$ & $\begin{array}{l}(2-7)-(2- \\
6)-(3-8)\end{array}$ & $9-6-5$ & $7-3-4$ \\
\hline \multicolumn{7}{|l|}{ Fifth Limb } \\
\hline no. of br. on the 3 endites & $\begin{array}{l}(2-4)-(2- \\
4)-(4,5,7)\end{array}$ & $\begin{array}{l}(2,3)-(3- \\
5)-(4,5,8)\end{array}$ & $\begin{array}{l}(2-4)-(2- \\
5)-(3-8)\end{array}$ & $\begin{array}{l}(1-5)-(3- \\
7)-(4-10)\end{array}$ & $3-5-8$ & $\begin{array}{c}3-(4,5)- \\
(5,7)\end{array}$ \\
\hline 3rd exop. seg., no. of br. on each lobe & $(2,3)-2$ & $(2,3)-2$ & $(1-3)-2$ & $(1-4)-(1-3)$ & $3-2$ & $3-2$ \\
\hline 4 th +5 th segs. no. of br. & $3-5$ & $5-7$ & $3-5$ & $3-5$ & 5 & 4,8 \\
\hline \multicolumn{7}{|l|}{ Sixth Limb } \\
\hline no. of epipodial br. & 2 & 2,3 & 1,2 & 1,2 & 2 & 2 \\
\hline no. of br. on the 4 endites & $\begin{array}{c}(2-4)- \\
(3,4)-(3,4)- \\
(1-3)\end{array}$ & $3-3-(3,4)-$ & $\begin{array}{c}(2,3)-(1- \\
3)-(1-4)- \\
(2,3)\end{array}$ & $\begin{array}{c}(1-3)-(1- \\
4)-(2-4)- \\
(2-4)\end{array}$ & $3-2-4-3$ & $\begin{array}{c}3-(2,3)- \\
(3,4)-3\end{array}$ \\
\hline end-seg., no. of br. & $6,7,9$ & $6-8$ & $6-8$ & $6-8$ & 7,8 & 7,8 \\
\hline \multicolumn{7}{|l|}{ Seventh limb } \\
\hline no. of terminal cleaning br. & $2-2$ & $3-3$ & $2-2$ & $(2,3)-(2,3)$ & $2-2$ & $3-3$ \\
\hline no. of prox. cleaning br. & $(1,2)-2$ & $(1,2)-2$ & $(1,2)-(1,2)$ & $(1-3)-(1-3)$ & $1-1$ & $(1,2)-(1,2)$ \\
\hline no. of comb-teeth & $\begin{array}{c}(9,11,13)- \\
(0,2)\end{array}$ & $\begin{array}{c}(7,9,11,14)- \\
(0,3,5)\end{array}$ & $\begin{array}{c}(1-3,13)-(1- \\
4,9)\end{array}$ & $(1-13)-(2-9)$ & $7-2$ & $7,9-2$ \\
\hline Furca, no. of main claws & 4 & 4 & 3,4 & 3,4 & 3 & 3,4 \\
\hline Furca, no. of secondary claws & 5,6 & 2,6 & $2-4$ & 2,3 & 3 & 3,5 \\
\hline Copulatory limbs long or short & Short & 一 & Long/short & - & Short & 一 \\
\hline
\end{tabular}

abs.: absent; br.: bristles; d: dorsal; dist.: distal; endop.: endopodite; ex.: exopod; fil.: filaments; no.: number; prox.: proximal; seg.: segment; sens: sensory; unk.: unknown; v: ventral. 
TABLE 11: Summary of characteristics of juvenile males and females of Rutiderma tridens. (Only juvenile specimens have been found for this species and so it was not included with adult females or males.)

\begin{tabular}{|c|c|c|}
\hline Sex & male & female \\
\hline Maturity & A-1 & A-1 \\
\hline Shell length, mm & 1.32 & 0.87 \\
\hline Surface with ribs (r) or smooth (s) & $\mathrm{r}$ & $\mathrm{r}$ \\
\hline Incisur shallow or deep & *Shallow & *Shallow \\
\hline No. of medial br. on rostrum (infold) & 5 & $(3-5)$ \\
\hline \multicolumn{3}{|l|}{ 1st antenna } \\
\hline no. of br. 2nd seg., d-la & $1-1$ & $1-1$ \\
\hline no. of br. 3rd seg., d.-v & $1-1$ & $1-1$ \\
\hline no. of br. 4 th seg., d.-v & $1-2$ & $1-2$ \\
\hline no. of fil. on sens. br. & 2 & 2 \\
\hline no. of fil. on b-br. & 0 & 0 \\
\hline no. of fil. on c.-br. & 0 & 0 \\
\hline no. of fil. on g.-br. & 1 & 1 \\
\hline \multicolumn{3}{|l|}{ 2nd antenna } \\
\hline endop. no. of br. on 1st seg. & 4 & 3 \\
\hline endop. no. of br. on 2 nd seg. & 3 & abs. \\
\hline endop. no. of br. on 3rd seg. & 2 & abs. \\
\hline \multicolumn{3}{|l|}{ Mandible } \\
\hline basale, no. of d. br. mid.-dist. & 2 & 2 \\
\hline basale, no. of thin v. br. & $* 5$ & $* 5$ \\
\hline basale, no. of stout v. br. & $* 2$ & $* 2$ \\
\hline basale, total no. of v. br. & 7 & 7 \\
\hline 1st end. seg., no. of v. br. & $(3-4)$ & $(3-4)$ \\
\hline 2nd end. seg., no. of d. br. & 3 & 3 \\
\hline 2nd end. seg., no. of v. br. excl. cl. & 2 & 2 \\
\hline 3rd end. seg., no. of br. excl. cl. & 7 & 7 \\
\hline Maxilla, no. of br. on the 3 endites & ? & $?$ \\
\hline \multicolumn{3}{|l|}{ 5th limb } \\
\hline no. of br. on the 3 endites & $?$ & $?$ \\
\hline 1st ex. seg., no. of primary teeth & 4 & 4 \\
\hline 2nd ex. seg., no. of teeth & 3 & 3 \\
\hline 2nd ex. seg., + or - bi-or trifurcate teeth & + & + \\
\hline 3rd exop. seg., no. of br. on each lobe & $3-2$ & $3-2$ \\
\hline 4 th +5 th segs. no. of br. & 5 & 5 \\
\hline \multicolumn{3}{|l|}{ 6th limb } \\
\hline no. of epipodial br. & 2 & 2 \\
\hline no. of br. on the 4 endites & $3-2-(1-2)-1$ & $3-2-(1-2)-1$ \\
\hline end-seg., no. of br. & 6 & 6 \\
\hline \multicolumn{3}{|l|}{ 7th limb } \\
\hline no. of terminal cleaning br. & $2-2$ & $2-2$ \\
\hline no. of prox. cleaning br. & $2-2$ & $2-2$ \\
\hline no. of comb-teeth & $8-2$ & $5-2$ \\
\hline Furca, no. of main claws & 3 & 3 \\
\hline Furca, no. of secondary claws & 3 & 3 \\
\hline Lateral Eye, + or - & + & + \\
\hline Specimen Number, \# = USNM & \#152829 & \#156686 \\
\hline
\end{tabular}

abs.: absent; br.: bristles; d: dorsal; dist.: distal; endop.: endopodite; ex.: exopod; fil.: filaments; no.: number; prox.: proximal; seg.: segment; sens: sensory; unk.: unknown; v: ventral, *: after Poulsen [10]. 


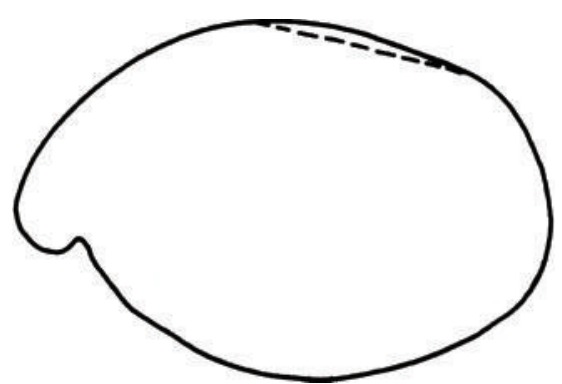

(a)

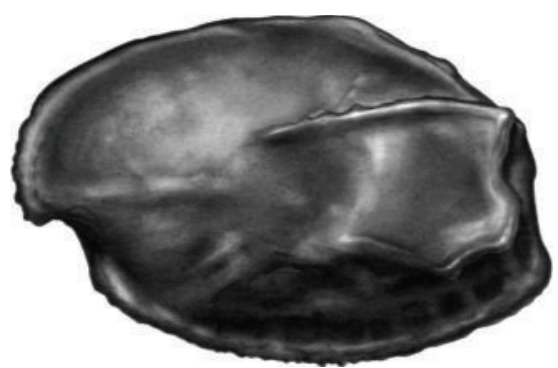

(c)

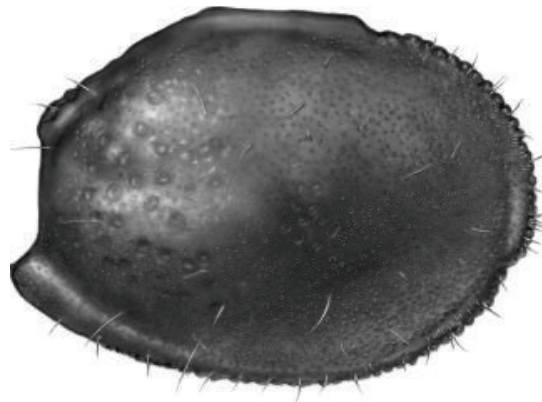

(e)

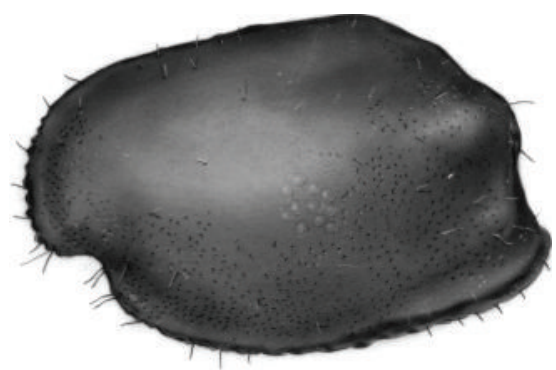

(g)

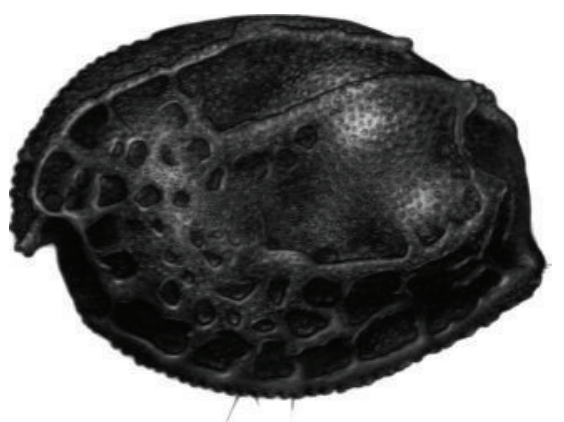

(b)

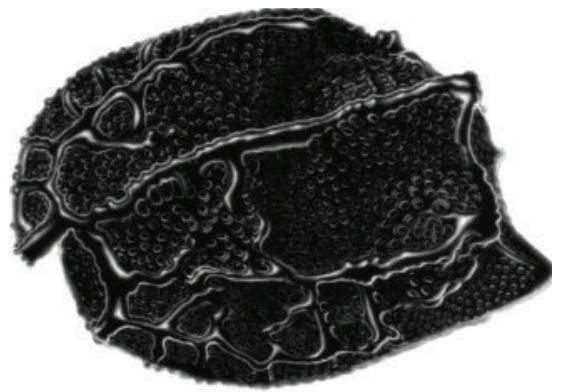

(d)

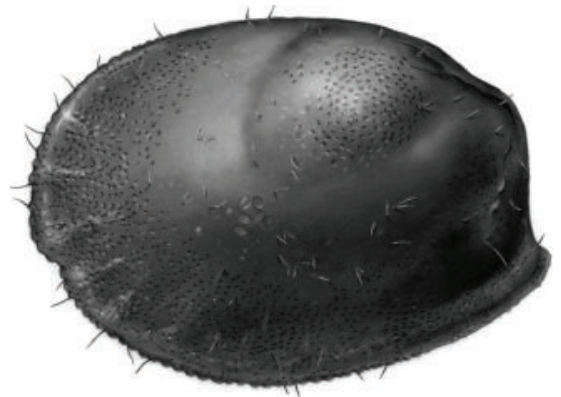

(f)

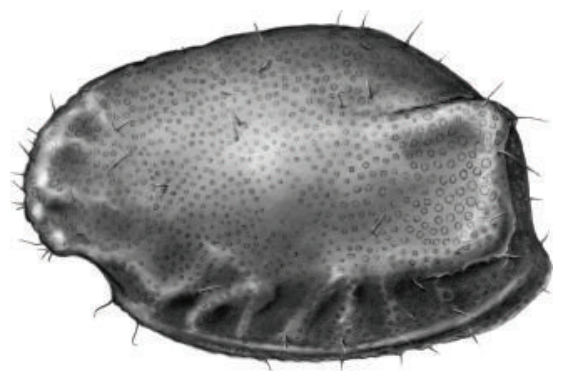

(h)

Figure 2: (a) Metaschisma nex, female, $1.49 \mathrm{~mm}$. (b) Rutiderma apex, ovigerous female, $1.18 \mathrm{~mm}$. (c) R. apex, male, $1.29 \mathrm{~mm}$. (d) R. arcuatilis, female, $1.02 \mathrm{~mm}$. (e) $R$. arx, female, $1.43 \mathrm{~mm}$. (f) $R$. arx, ovigerous female, $1.42 \mathrm{~mm}$. (g) $R$. arx, male, $1.32 \mathrm{~mm}$. (h) $R$. arx, male, $1.41 \mathrm{~mm}$.

to $1.05 \mathrm{~mm}$, compared to from 1.18 to $1.25 \mathrm{~mm}$ for $R$. apex. The rostral infold of the female $R$. judayi bears a row of 7 bristles compared to from 10 to 12 on $R$. apex. The vertical rib at the posterior end of the alar process on the carapace of the female $R$. lomae [58] has a space near middle, whereas it is continuous on $R$. apex. Adult males of $R$. lomae and $R$. apex are difficult to separate, except that the middle part of the vertical rib of the carapace of $R$. apex does appear to be better developed (see Kornicker and Myers [14, page 15, for $R$. lomae]). The caudal process of the carapace of the female $R$. chessi projects farther than that of $R$. apex, the c-bristles of the second and third endopodial segments of the mandible are more elongate, and the Bellonci organ has a pointed rather than a rounded tip. The ridges of the carapace of the female $R$. rostrata are less well defined than those of $R$. apex, and the c-bristle of the second endopodial segment of the 
female mandible of $R$. rostrata has a long produced tip that is absent on $R$. apex. The ribs of the carapace of $R$. apex resemble those of $R$. hartmanni from the Gulf of Panama, but the carapace of $R$. apex is slightly larger and the caudal process has less posterior projection; the anterior ridge of the infold of the caudal process is more concave posteriorly on $R$. hartmanni, and the c-bristle of the second endopodial segment of the female mandible of $R$. hartmanni bears a small terminal extension that is absent on $R$. apex. The lateral ribs of $R$. rotunda are evenly rounded posteriorly, not indented like the vertical rib of $R$. apex, and each lamella of the furca of $R$. rotunda bears 3 primary claws compared to 4 on $R$. apex (Kornicker and Harrison-Nelson [51, page 43]). According to molecular analysis, Oakley [65] reported that Rutiderma apex is the sister group to Euphilomedes (Philomedidae) (Kornicker and Harrison-Nelson $[51$, pages 43,45$])$.

\subsection{Rutiderma arcuatilis}

5.2.1. Kornicker [16] (Figure 2(d)). Rutiderma arcuatile Kornicker [16, pages 11-13, 25, 67-70, Table 1, Figures 39, 40], Kornicker [33, Table 3], Rutiderma arcuatilis Kempf [68, page 668], Kornicker [48, page 84 (compares to R. vox)], Kornicker and Harrison-Nelson [43, Tables 8, 9].

Holotype. USNM 158212, adult female.

Type Locality. East side of Bolongo Bay, St. Thomas Island, U.S. Virgin Islands. $18^{\circ} 18^{\prime} 59^{\prime \prime} \mathrm{N}, 64^{\circ} 53^{\prime} 4^{\prime \prime} \mathrm{W}$, intertidal.

Distribution. Northwest Atlantic: Virgin Islands: St. Croix and St. Thomas Islands.

Habitat. Benthic, intertidal, shifting sands by rocks; planktonic near bottom, $9 \mathrm{~m}$, outer reef.

Life History and Ontogeny. Female, 3 eggs.

Stomach Contents. Copepods.

Comparisons. R. arcuatilis is closely related to $R$. dinochelata. On $R$. arcuatilis the curvature of the list of the caudal process has a low angle with the ventral margin. On both $R$. dinochelata and $R$. mortenseni (only male known), the curvature of the list forms about a $45^{\circ}$ angle with the ventral margin. Also, on both of these species, bristles are present on each end of the curved list, whereas $R$. arcuatilis bears several bristles along the list (Kornicker [16, page 70]).

5.3. Rutiderma arx Kornicker, 1992 (Figures 2(e)-2(h)). Rutiderma arx Kornicker, [49, pages 124-140, Key to instars of Rutiderma: 123, Tables 1, 2, 13-17, Figures 72-79, 80], Kornicker [37, page 114], Kornicker and Thomassin [52, pages 73-83, Figures 48-57, Table 1, Appendix, (supplementary description of adult female) (Northeastern end of Mozambique Channel, Indian Ocean)], Kornicker and Harrison-Nelson [43, pages 427, 456-457 (comparison of development of $R$. darbyi to $R$. arx), Tables $1,4,7,8,9]$.

Holotype. MNHN Os 272, ovigerous female, Museum National d'Histoire Naturelle, Paris, France.

Type-locality. Station 101-DS, 8 Apr 1977, NW le du Lys, Glorioso Islands, $11^{\circ} 25^{\prime} 42^{\prime \prime} \mathrm{S}, 47^{\circ} 19^{\prime} 30^{\prime \prime} \mathrm{E}$, depth $26 \mathrm{~m}$.
Distribution. Glorioso Islands, depth 24 to $26 \mathrm{~m}$. Madagascar, depth: on reef flat to $31 \mathrm{~m}$.

Habitat. Benthic (infauna). Gravelly sand, sedimentary pocket with nodules of melobesians in large ripples, coarse sediment among coral patches.

Life history and ontogeny. Adult male and female, ovigerous female (4 eggs), instar I, instar II, male instar III, male instar.

Gut content. One specimen with two harpacticoid copepods, and fragment of (?) nematode.

Comparisons. Rutiderma arx differs from both $R$. leloeuffi and $R$. tridens in the female carapace having a smaller rostrum and in the terminal claw of the second endopodial segment of the female mandible not having a pronounced tip. The infold of the caudal process of $R$. tridens has 3 "teeth" along the dorsal margin of the "pocket" that are absent on $R$. arx, and the carapace of $R$. tridens bears lateral ribs absent of $R$. arx. Rutiderma irrostrata differs from $R$. arx in having a carapace with lateral ribs, and in having marginal teeth on the 3 lobes of the large flat tooth forming the second exopodial segment of the fifth limb. The carapace of $R$. arx differs from that of $R$. compressa and $R$. normani in lacking lateral ribs. Only the male is known of R. fusca; the posterior edge of the alar process on the carapace of that species bears a backward pointing triangular process at the ventral and dorsal ends that is not present on the male of $R$. arx. The male $R$. arx (length $1.30-1.34 \mathrm{~mm}$ ) is longer than the male $R$. fusca (length $1.10 \mathrm{~mm}$ ) (Kornicker [49, page 136]).

5.4. Rutiderma chessi Kornicker and Myers [14] (Figure 3(a)). Rutiderma chessi Kornicker and Myers [14, pages 2, 3, Key to species of adult females and males of Rutiderma of southern California, 31-34, Figures 19, 20], Kornicker [48, page 84], Kornicker and Harrison-Nelson [43, Tables 8, 9].

Holotype. USNM 158280, ovigerous female.

Type Locality. San Clemente Island, station D-3.

Distribution. Northeast Pacific: off California, USA: San Clemente Island.

Life History and Ontogeny. Female, 4 eggs.

Comparisons. The rib structures on the carapace of $R$. chessi differ from those of other species of Rutiderma in southern California. The species whose carapace resembles it most closely is R. judayi, which is smaller, has 3 instead of 2 main horizontal ribs on each valve, has more processes along the posterodorsal margin, and has a less acute caudal process. The main claws on the second and third endopodial segments of the female mandible are longer and more slender than those on other species of Rutiderma in the area (Kornicker and Myers [14, page 34]).

5.5. Rutiderma cohenae Kornicker [16] (Figure 3(b)). Rutiderma cohenae Kornicker [16, pages 4, 1-13, 15, 25, 26, 6267, 85, Table 1, Figures 3, 36-38], Kornicker [33, Table 3], Cohen [20, pages 322, 331], Kornicker [48, page 84], Kornicker and Harrison-Nelson [43, Tables 8, 9], Kornicker et al. [56, Table 1], Harrison-Nelson et al. [53, page 874] .

Holotype. USNM 158359, ovigerous female.

Type Locality. San Salvador, Bahamas; San Salvador Island, dump reef, depth from subtidal to $4 \mathrm{~m}$. 


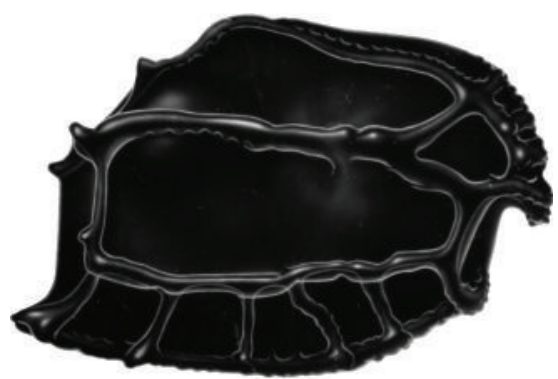

(a)

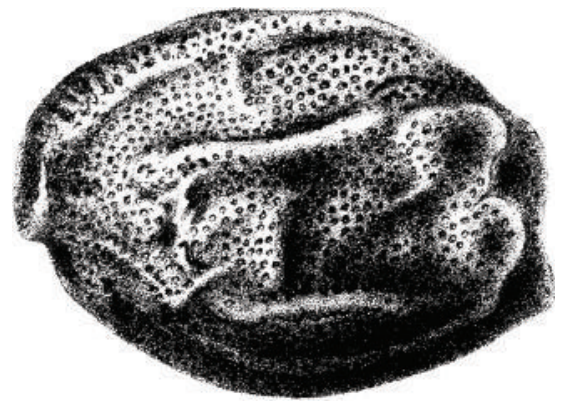

(c)

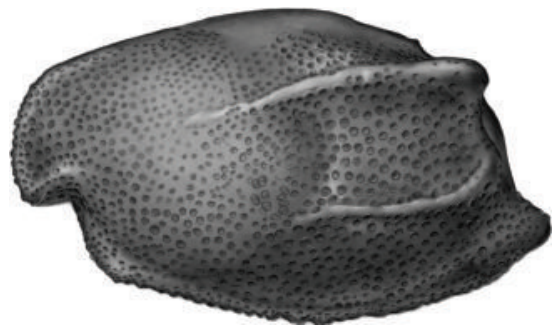

(e)

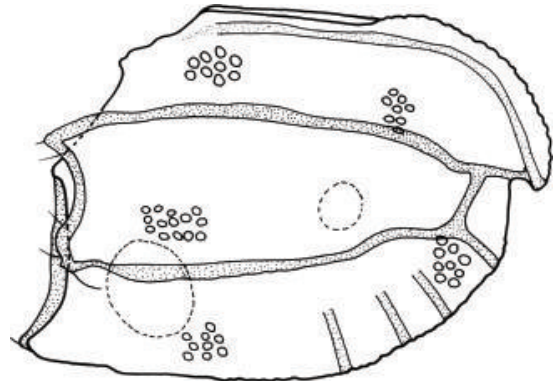

(g)

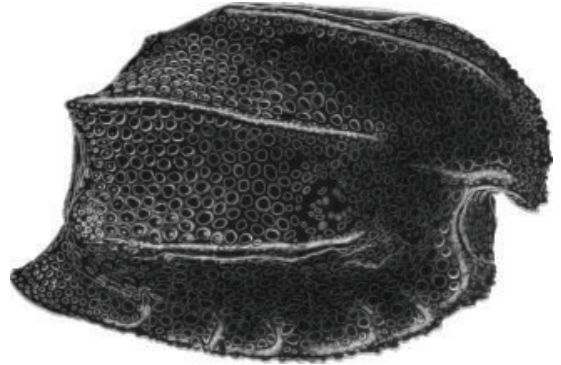

(b)

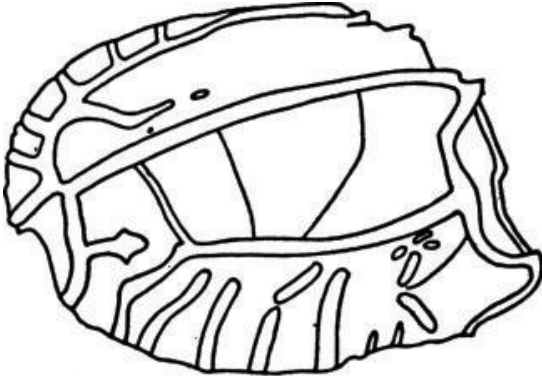

(d)

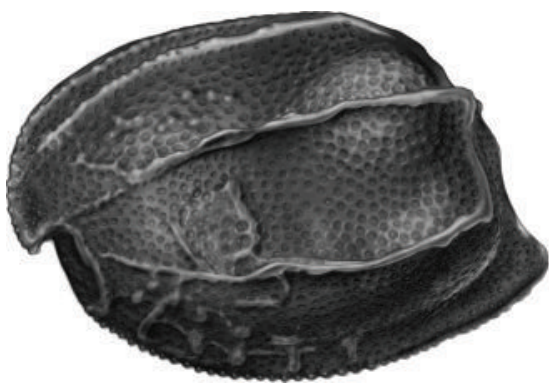

(f)

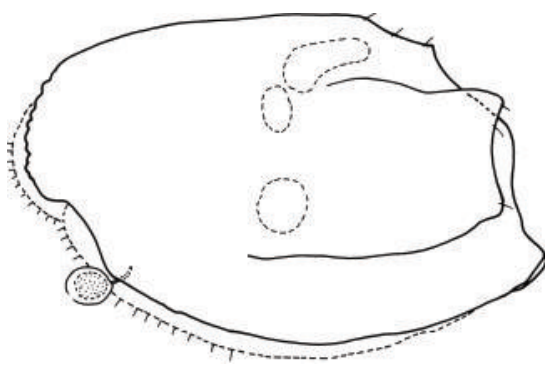

(h)

Figure 3: (a) Rutiderma chessi, female, $1.21 \mathrm{~mm}$. (b) R. cohenae, female, $1.24 \mathrm{~mm}$. (c) $R$. compressa, female, $1.5 \mathrm{~mm}$. (d) $R$. dinochelata, female, $1.18 \mathrm{~mm}$. (e) R. darbyi, male, $1.12 \mathrm{~mm}$. (f) R. darbyi, female, $1.28 \mathrm{~mm}$. (g) R. dux, female, $1.11 \mathrm{~mm}$. (h) R. dux, male, $1.05 \mathrm{~mm}$.

Distribution. Carrie Bow Cay, Belize; Fleming Key, Key West, Florida; Virgin Islands.

Habitat. Benthic from subtidal to $4 \mathrm{~m}$; sand in Thalassia beds; spur-and-groove, outer fore-reef slope.

Life History and Ontogeny. Adult female, 4 eggs.

Comparisons. The elongate finger-like extension of the tip of the stout terminal claw of the second endopodial segment of the mandible and the small, slender, unringed, posterior bristle on the endopodite of the second antenna of the adult female distinguishe $R$. cohenae from the previously described species. A few species have one of the above characters but not both. The new species differs from $R$. mortenseni Poulsen [10, page 38], of which only the male is known, in the morphology of the infold of the caudal process and in having fewer bristles on the rostral infold (Kornicker[16, page 67]).

5.6. Rutiderma compressa Brady and Norman [55] (Figure 3(c)). Rutiderma compressa Brady and Norman $[55$, pages $5,6,8,9,17,18,38,43,623,673-675$, Plate 
58, Figures 9-16 (holotype: unknown; type locality: "one of these from off the Cap de Penas (Bay of Biscay))], the other from the Fosse de Cap Breton," Müller [46, page 35], Klie [5, pages 404-405, 406 (identification of African specimens needs verification)], Kornicker [6, page 236], Hartmann [7, page 39 (identification of Red Sea specimens needs verification)], Poulsen [10, pages $6,8,9,17,38$, 43], Hartmann-Schröder and Hartmann [8, pages 25, 30, 33, Table 3], Hartmann [26, pages 183, 192: Figure 111c, (according to G. W. Müller 1908), page 201: Figure 115b] Puri [69, page 475], Hartmann [70, page 237], Kornicker [13, pages 30, 646, 657, 675], Kornicker [54, page 43 (compares to $R$. leloeuffi)], Kornicker and Caraion [47, pages 2, 54, 65, 66 (compares to $R$. tridens)], Kornicker [16, page 25 (mentions)], Kornicker [63, page 3 (mentions)], Kornicker and Iliffe [71, page 43], Kornicker [48, page 84 (compares to R. vox)], Kornicker [49, page 136], Kornicker and Harrison-Nelson [43, page 429 (mentions)].

(?) Rutiderma compressa. Müller [59, pages 53, 92, 93, Plate 7: Figures 1-13] (questionably referred to $R$. tridens by Kornicker and Caraion [47, pages 60, 65: "The carapace of $R$. tridens resembles that of the female of $R$. compressa from South Africa] illustrated by Müller [59, Plate 7:1]). Müller's specimen has been put into synonymy of $R$. tridens with a question, because the first antenna illustrated by Müller [59, Plate 7:2] does not show a lateral bristle on the second segment, but this could be because the illustration is a medial view of the limb. The first, third, and fourth endites of the sixth limb illustrated by Müller [59, Plate 7:12] have a different number of bristles than do the same endites of the two specimens of $R$. tridens described herein, but this could be the result of intraspecific variability.").

Rutiderma (Rutiderma) compressa. Kornicker [6, pages 236, 237 (compares to $R$. dinochelata)], Hartmann in Hartmann-Schröder and Hartmann [8, page 328].

(?) Rutiderma africana. Puri [69, page 484] (Puri credits $R$. africana Müller to Klie [5, pages 404-448], but $R$. africana is not described or mentioned in the Klie [5] or Müller publications. Puri probably meant $R$. compressa instead of $R$. africana because Klie [5] lists $R$. compressa between Cypridina dorsoserrata Müller and Philomedes africana Klie, and Puri [69], lists R. africana Müller between Cypridina dorsoserrata Müller and Philomedes africana Klie).

(?) Rutiderma cf. compressa. Hartmann-Schröder and Hartmann [72, pages 48, 56, 77], Hartmann and HartmannSchröder 1975:360 [73] (identification of African specimens needs verification).

Rutiderma cf. compressa Brady and Norman [55], Hartmann [70, page 237].

Not Rutiderma compressa. Hartmann [60, page 328] (adults $=R$. gerdhartmanni Kornicker [13, pages 648-649, 673 ] juveniles $=R$. species A).

Holotype. Unknown.

Type Locality. One collection off the Cap de Peñas (Bay of Biscay), the other from the Fosse de Cap Breton, depth $150 \mathrm{~m}$.

Distribution. Northeast Atlantic: Bay of Biscay; Cabo de Penas, Spain; Fosee Cap Breton, France (fide Klie [5]).
(?) Southeast Atlantic: Lüderitz Bay, South West Africa; Simonstown, Atlantic coast of South Africa near Cape of Good Hope. (?) Indo-West Pacific: Al Ghardaqah, Egypt, Red Sea; Tongaat, coast of South Africa; Xai Xai, Moçambique, Mozambique.

Habitat. Benthic; $150 \mathrm{~m}$; (?) from 1 to $2 \mathrm{~m}$, subtidal; (?) shallow, sand; (?) rock and gravel beach, hummock-algae, tropical littoral; (?) rock-dwelling.

Life History and Ontogeny. Female, A-1 female, juvenile.

Comparisons. The incisur on $R$. compressa is less distinct and the rostrum and caudal process is less prominent than on $R$. leloeuffi. The incisur forms a right angle, unlike $R$. licina, which has a deep incisur with overhanging rostrum. It is distinguished from $R$. ovata in that the carapace does have a projecting posteroventral caudal process. Rutiderma compressa differs from $R$. gerdhartmanni in the morphology of the first antenna. While $R$. gerdhartmanni has a bristle on the second segment and 2 bristles on the third segment, $R$. compressa lacks a bristle on the second segment and has just 1 on the third segment. Rutiderma compressa can also be differentiated from $R$. gerdhartmanni in that $R$. compressa has numerous riblets between the anterodorsal rib and anterodorsal shell margin, while $R$. gerdhartmanni lacks these riblets.

5.7. Rutiderma darbyi Kornicker [16] (Figures 3(e)-3(f)). Rutiderma dinochelata Darby [74, pages 28, 56, 57, Plate 13: Figures 1-9, Plate 14: Figures 1b-7], Kornicker [13, pages 65, 74] (not Rutidema dinochelata Kornicker [6]).

Rutiderma darbyi Kornicker, Bradford [75, pages 141, 143, Figure 2] (deliberate nomen nudum).

Rutiderma darbyi. Kornicker [16, pages 2-7, 11-14, 17, 25, 36-47, 62, 85, Table 1, Figures 1, 18-24, Plate 1], Kornicker [63, page 25], Kornicker [33, Table 3], Cohen [20, pages 322, 331], Kornicker [48, page 84], Kornicker [37, page 114], Grabe et al. [17, page 698, Tables 2, 3], Kornicker and Harrison-Nelson [43, pages 426-471, Figures 2-20, Tables 1-9, key to stages of Rutiderma darbyi (males and females) page 456], Kornicker et al. [44, pages 82-83, Table 1, Figure 59], Grabe [18, pages 57, 59, 62, 67, Tables 2-5, Figure 4], Kornicker et al. [56, page 95, Table 1], HarrisonNelson and Kornicker [53, page 874].

Holotype. USNM 158003, ovigerous female.

Type Locality. Georgia continental shelf, $31^{\circ} 05^{\prime} \mathrm{N}$, $80^{\circ} 35^{\prime} \mathrm{W}, 26 \mathrm{~m}$.

Distribution. Continental shelf off North and South Carolina, Georgia, and Florida (Atlantic and Gulf of Mexico); also, Bahama Islands in vicinity of San Salvador Island, Andros Island, and in Crab Cay Cravasse, Exuma Islands. Known depth range from intertidal to $168 \mathrm{~m}$.

Life History and Ontogeny. Female, male, instars I-IV, from 2 to 4 eggs.

Parasites. Choniostomatid copepods Bradford [75, page 141], Kornicker [16, page 43].

Comparisons. The carapace of $R$. darbyi differs from known species in the study area in having several flat spinelike processes along the ventral edge of "pocket" in the caudal process of the left valve. They are absent on the right valve. The spine-like processes are generally visible when the whole specimen is viewed using transmitted light and an objective 
lens having a magnification of $10 x$ or $20 x$. The spine-like processes are present on juveniles and adults of both sexes (Kornicker [16, page 47]).

5.8. Rutiderma dinochelata Kornicker [6] (Figure 3(d)). Rutiderma (Rutiderma) dinochelatum Kornicker [6, pages 236238, Figures 46: 8A-B, 57: A-F, 58: A-D, 86: B,F,J].

Rutiderma (Rutiderma) dinochelata. Kornicker [6, page 297, Figure 86: B,F,J] (misspelling).

Philomedes lomae. Kornicker [6, pages 233, 234, Figures 46: 7A-B, 52: A-E, 53: A-D, 86: A,E.I], Poulsen [10, pages 15,17$]$ (recognizes specimens as male Rutiderma) not Juday [58], not Darby [74, page 28, Plates 13, 14] (= R. darbyi, new species).

Rutiderma dinochelata. Hartmann in HartmannSchröder and Hartmann [8, page 328] (mentions), McKenzie [9, page 66] (compares to R. judayi), Poulsen [10, pages 7, 8, 17, 18, 43], Kornicker [76, page 110], Kornicker [13, page 675], Kornicker [54, page 43] (compares to R. leloeuffi), Kornicker [31, page 8] (compares to R. sterreri), Kornicker [16, pages 11-12, 25-28, 70, Table 1, Figures 2, 10, 11], Kornicker [33, Table 3], Kornicker [48, page 84], Cohen [20, pages 322, 324, 325, 327, 330, 331, 332, 333], Kornicker and Harrison-Nelson [43, Table 9], Kornicker et al. [56, page 95, Table 1].

Holotype. USNM 122907, adult female.

Type Locality. Bimini area, Great Bahama Bank, depth from 1 to $20 \mathrm{~m}$.

Distribution. Northwest Atlantic: Bahama Islands: Bimini and Andros Islands. Belize: Carrie Bow Cay.

Habitat. Benthic, from intertidal to $30 \mathrm{~m}$; temperature about $29^{\circ} \mathrm{C}$, salinity: from $31-42 \%$; lagoon, back-reef $1.5 \mathrm{~m}$, sand and rubble zone; outer fore-reef, $30 \mathrm{~m}$, sand trough.

Life History and Ontogeny. Female, juvenile female.

Biology. Response to light (Kornicker [6, page 224]).

Comparisons. The carapace of the male $R$. dinochelata differs from that of $R$. flex in that the upper lateral rib does extend anteriorly to intersect the anterior edge of the rostrum and the lower lateral rib does extend anteriorly to midlength of the carapace. The species $R$. dinochelata differs from $R$. sterreri in that the tip of the Bellonci organ is pointed, not rounded. The list of the caudal process of the female $R$. vox is longer and more oblique than that of the female $R$. dinochelata. Rutiderma dinochelata is very similar to $R$. arcuatilis, but they differ in that the list of the caudal process on $R$. arcuatilis has a low angle with the ventral margin, but, in $R$. dinochelata, the curvature of the list forms about a $45^{\circ}$ angle with the ventral margin.

5.9. Rutiderma dux Kornicker [50] (Figures 3(g)-3(h), 4(a)). Rutiderma dux Kornicker [50, pages 22-28, Figures 13-16, (compares to R. normani), Table 1, Appendices 1-2].

Holotype. AM P45375, female with unextruded eggs.

Type Locality. Darwin, Australia, station JLB Darwin 302 and 305 combined (Station 302: Channel Island, 20 Aug 1982, mud; station 305 (same as station 304): East Point, 22 Aug 1982), both samples from intertidal washings of algae and substrate.
Distribution. Channel Island and/or East Point, Darwin, Australia, and Lizard Island, Great Barrier Reef, Australia.

Habitat. Mud.

Life History and Ontogeny. Adult male, A-1 male, female, ovigerous female (3 to 4 eggs), juvenile.

Comparisons. Rutiderma $d u x$ is close to $R$. normani Poulsen [10, page 22], and they could be conspecific. The female sixth limbs of the two species differ in that the ventral margin of the end segment of $R$. normani is straight (Poulsen $[10$, Figure $4 j])$, whereas the anterior 3 bristles on the end segment of $R$. dux are on a long projection. Also, Poulsen $[10$, pages 26,28$]$ described both the female and male furcae of $R$. normani as having 3 main claws and 3 secondary claws, whereas the female and male furcae of $R$. dux have 4 main claws and 2 secondary claws (Kornicker [50, page 28]).

5.10. Rutiderma exrex Kornicker in Kornicker and Thomassin [52] (Figure 4(b)). Rutiderma exrex Kornicker in Kornicker and Thomassin [52, pages 87-92, Figures 61-63, Table 1, Appendix], Kornicker and Harrison-Nelson [43, Tables 8, 9].

Holotype. Undissected adult female with large unextruded eggs; Muséum National d'Histoire Naturelle.

Type Locality. BT-227, “Oct 1969; southern Grand Récif, southern corner transect; close on outerslope; coral flagstone; depth $27 \mathrm{~m}$; bottom with nodules of melobesians or rhodoliths, algae dominant."

Distribution. Tuléar Reef Complex, SW Madagascar.

Habitat. Grand Récif, South Lovobé area; reef flat; boulder tract; front of a detrital embankment; SC (shovel). Nosy Vé Cay reef; west beach (seaward side); infralittoral; reef flat sand with ripples and scattered Phyllochaetopteridea mats; SC ( shovel). Coral flagstone; depth $27 \mathrm{~m}$; bottom with nodules of melobesians or rhodoliths, algae dominant.

Life History and Ontogeny. Adult female.

Comparisons. Rutiderma exrex is quite similar to $R$. rex and they could be conspecific. The carapace of $R$. exrex differs from that of $R$. rex in three characters: (1) the ribs and riblets of $R$. rex have high relief, whereas, those of $R$. exrex are barely visible, (2) the ridge forming the anterodorsal edge of the pocket of the infold of the caudal process is slightly convex posteriorly on $R$. rex and slightly concave on $R$. exrex, but there is some variability in this character; and (3) the posterior edge of the alar process on the outer surface of each valve as well as the posterodorsal edge of the valves of $R$. rex bears small tubercles that are either absent or much smaller in $R$. exrex. Also, the length of the female carapace of $R$. rex is from 0.90 to $0.92 \mathrm{~mm}$ (3 specimens), whereas the length of the female $R$. exrex is from 0.99 to $1.19 \mathrm{~mm}$ ( 3 specimens), but the difference could be the result of intraspecific variability. The carapace of $R$. exrex differs from those of $R$. arx and $R$. ferax in having a caudal process with less posterior projection, and the ridge forming the anterodorsal edge of the pocket of the infold of the caudal process forms a $45^{\circ}$ angle in $R$. exrex but forms a shallow arc at a much lower angle with the ventral edge of the caudal process in $R$. arx and R. ferax. Also, the ribs and riblets on the outer surface of the carapace of $R$. ferax have much greater relief than those of $R$. exrex. The tip of the dorsal margin 


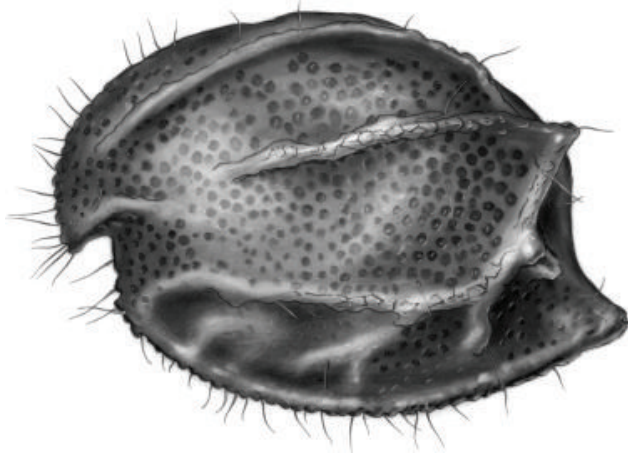

(a)

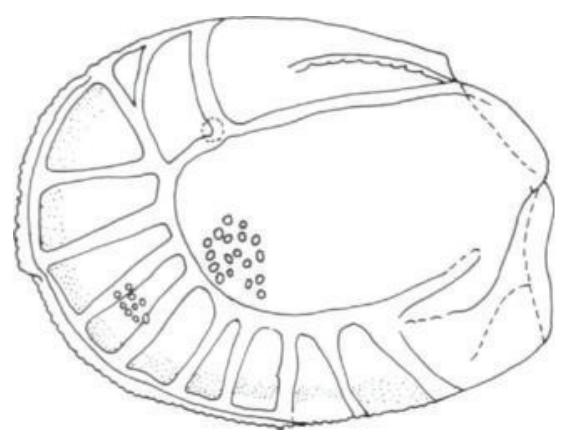

(b)

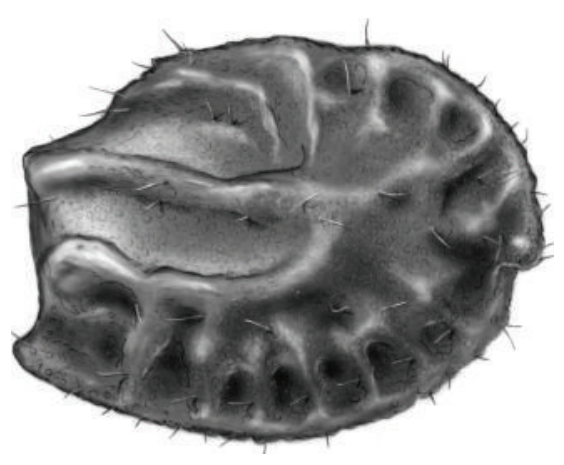

(c)

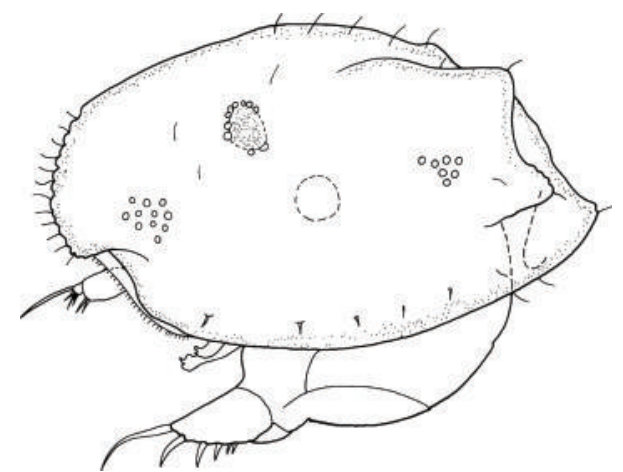

(d)

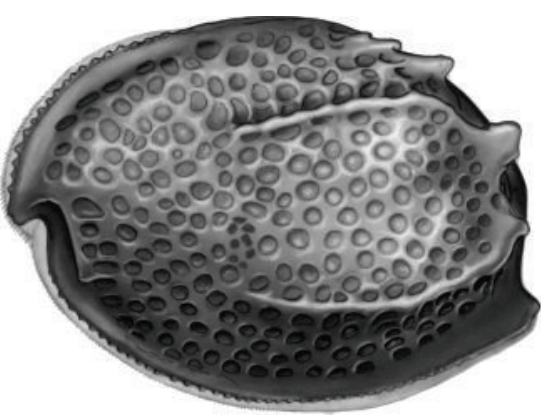

(f)

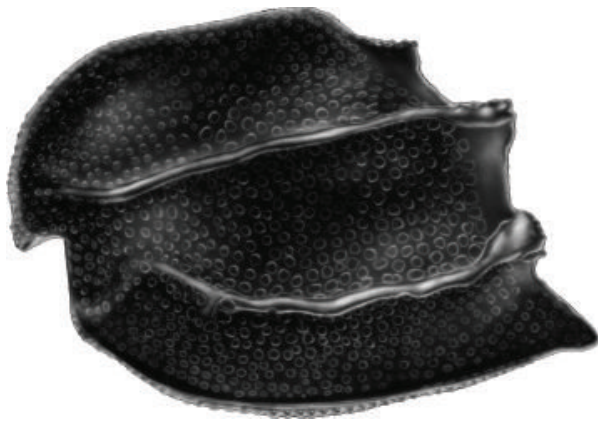

(h)

Figure 4: (a) Rutiderma dux, A-1 male, $0.99 \mathrm{~mm}$. (b) R. exrex, female, $0.99 \mathrm{~mm}$. (c) R. ferax, female, $1.16 \mathrm{~mm}$. (d) $R$. flex, male, $1.21 \mathrm{~mm}$. (e) R. fusca, male, $1.10 \mathrm{~mm}$. (f) R. gerdhartmanni, female, $1.38 \mathrm{~mm}$. (g) R. gerdhartmanni, male, $1.48 \mathrm{~mm}$. (h) R. gyre, female, $1.10 \mathrm{~mm}$. 
of the claw-like c-bristle of the third endopodial segment of the mandible of $R$. arx has a small projecting tooth that is absent on $R$. exrex. The proximal and middle lobes of the large flat second exopodial segment of the fifth limb have 1 or 2 marginal cusps in $R$. ferax, but they are without cusps in $R$. exrex. The Y-sclerites of $R$. ferax and many specimens of $R$. arx are without a ventral branch, which is present in $R$. exrex. The female carapace of $R$. exrex is smaller than that of $R$. arc (Kornicker and Thomassin [52, page 92]).

\subsection{Rutiderma ferax Kornicker in Kornicker and Thomassin} [52] (Figure 4(c)). Rutiderma ferax Kornicker in Kornicker and Thomassin [52, pages 83-87, Figures 58-60, Table 1, Appendix], Kornicker and Harrison-Nelson [43, Tables 8, 9].

Holotype. Adult female holotype, Muséum National d'Histoire Naturelle, Paris.

Type Locality. Madagascar, BT-836 (Grand Récif; Petite Vasque 2 (residual pool 2); Sediment Collected (bag)).

Distribution. Type locality only.

Habitat. Grand Récif, Residue Pools of Reef Flat, sand, collected with a SC (shovel).

Life History and Ontogeny. Adult female.

Comparisons. Rutiderma ferax is very similar to R. rex. The main difference noted is in the infold of the caudal process of the carapace: the ridge dorsal to the pocket is straight of slightly convex and forms a $45^{\circ}$ angle in $R$. rex (see Kornicker [49, Figure s 82b,c]) and is a shallow concave arc more-or-less parallel to the ventral edge of the caudal process in $R$. ferax. The length of the carapace of the female $R$. ferax is $1.16 \mathrm{~mm}$, compared to from 0.90 to $0.92 \mathrm{~mm}$ (three specimens) for R. rex (Kornicker [49, page 142]). The caudal process has greater posterior projection in $R$. ferax. The anterodorsal and anteroventral infolds of $R$. rex each bears 4 or 5 bristles compared to 8 on $R$. ferax. The endopodite of the second antenna of $R$. ferax bears a small posterior spine absent on $R$. rex. The proximal and middle lobes of the second exopodial segment of the fifth limb of $R$. ferax bear 1 or 2 marginal cusps absent on $R$. rex. The Y-sclerite of $R$. rex bears a ventral branch absent on $R$. ferax (Kornicker and Thomassin [52, page 87]).

5.12. Rutiderma flex Kornicker in Kornicker et al. [56] (Figure 4(d)). Rutiderma flex Kornicker in Kornicker et al. [56, pages 91-95, Figures 48-51, Tables 1, 2].

Holotype. USNM 1021462, adult male.

Type Locality. Conch Sound Blue Hole, Andros Island, Great Bahama Bank.

Distribution. Type locality only.

Habitat. Sediment at $25 \mathrm{~m}$ depth about $365 \mathrm{~m}$ inside cave. Life History and Ontogeny. Adult male.

Comparisons. The carapace of the male $R$. flex differs from that of $R$. dinochelata in that the upper lateral rib does not extend anteriorly to intersect the anterior edge of the rostrum, and the lower lateral rib does not extend anteriorly to midlength of the carapace. The structure of the posterior edge of the shelf in the anterior part of the pocket of the infold of the caudal process of $R$. flex differs from those of $R$. darbyi (Kornicker [16, Figure 23b]) and R. schroederi (Kornicker and Iliffe [77, Figure 53c,d (female)]). The infold of the rostrum of $R$. flex bears a row of 7 bristles compared to 12 on $R$. mortenseni (Poulsen [10, Figure 11b]). The adult male of $R$. flex is larger than that of $R$. licina Kornicker [16], the furca bears 1 or 2 instead of 3 secondary claws, and the basis of the mandible bears 4 instead of 6 bristles near the ventral margin (Kornicker et al. [56, page 95]).

5.13. Rutiderma fusca Poulsen [10] (Figure 4(e)). Rutiderma fusca Poulsen [10, pages 7, 8, 11, 14, 17, 38, 41-44, Figure 12], Kornicker [13, pages 70, 80], Cohen and Kornicker [78, pages 21, 26], Kornicker [49, page 136], Kornicker et al. [44, Tables 8,9].

Holotype. Male, Zoological Museum, University of Copenhagen.

Type Locality. "Guardaga," Red Sea; surface (perhaps locality is Al Ghardaqah, Egypt).

Distribution. Type locality only.

Life History and Ontogeny. Male.

Comparisons. The Bellonci organ of $R$. fusca is pointed, whereas the Bellonci organ of $R$. rex is broadly rounded. Rutiderma fusca differs from the male $R$. arx in that the posterior edge of the alar process on the carapace of $R$. fusca species bears a backward pointing triangular process at the ventral and dorsal ends not present on the male R. arx.

5.14. Rutiderma gerdhartmanni Kornicker [13] (Figures 4(f)-4(g)). Rutiderma compressa Hartmann et al. [60, page 328 (part: adults)], Hartmann-Schröder and Hartmann [8, pages 25, 30,33,41, 45 (adults only)] (not Brady and Norman [55]).

Rutiderma gerdhartmanni. Kornicker [13, pages 25, 29, $47,60,70,74,80,646-657,673-675,678$, Figures 406, 408410], Kornicker [54, page 43 (nom. nud.)], Hartmann and Petersen [79, page 228 (location of types Zoologischen Museums der Universität Hamburg)], Kornicker [30, Table 15], Kornicker [48, page 84], Parker [39, Table 1], Kornicker and Harrison-Nelson [43, Tables 8, 9].

Holotype. Gravid female labeled "Holotype," (no. 27297 includes all specimens from Chile identified by G. Hartmann as Rutiderma compressa), Hamburg Zoological Museum.

Type Locality. Bahia Ingles, Chile, $41^{\circ} 48^{\prime} \mathrm{S}, 75^{\circ} 53^{\prime} \mathrm{W}$, $12 \mathrm{~m}$.

Distribution. Type locality only.

Habitat. Benthic; $12 \mathrm{~m}$; sand-mud and gravel.

Life History and Ontogeny. Female, male, 4 eggs.

Comparisons. The specimens identified by Hartmann as $R$. compressa have been assigned to a new species primarily because of the sixth limb on which the anterior part of the end segment forms a projecting process bearing 3 bristles. The sixth limb of $R$. compressa was not described in the original description by Brady and Norman [55] but was illustrated by Müller [59] from a specimen collected near South Africa. His figure shows the anterior part of the end segment of the sixth limb to be only very slightly separated from the posterior part. I have not examined either the specimens described by Brady and Norman or that described by Müller, and it is possible that they do not belong to the same species; however, both Brady and Norman and Müller illustrated the first antenna of a female. These illustrations 
show the lack of a lateral bristle on the second segment and only 1 dorsal bristle on the third segment. The first antenna of $R$. gerdhartmanni bears a lateral bristle on the second segment and 2 dorsal bristles on the third segment. I do not, however, rely strongly on those differences in the first antennae, because bristles may have been overlooked by Brady and Norman and Müller, or they may have described and illustrated second antennae from juveniles. The carapace illustrated by Brady and Norman bears numerous riblets between the anterodorsal rib and the anterodorsal shell margin. These are not present on $R$. gerdhartmanni. The length of the carapace of the female $R$. gerdhartmanni is in the order of from $1.35 \mathrm{~mm}$ to $1.39 \mathrm{~mm}$; the length of $R$. compressa given by Brady and Norman [55, page 674] was $1.5 \mathrm{~mm}$; the length of the specimen identified as $R$. compressa by Müller was given by him as $1.6 \mathrm{~mm}$ [59, page 92] (Kornicker [13, page 657]).

5.15. Rutiderma gyre Kornicker [16] (Figures 4(h), 5(a)). Rutiderma gyre Kornicker [16, pages 4-8, 10-13, 15, 25, 54-62, 80, Table 1, Figures 30-35, Plate 3], Kornicker [33, Table 3], Kornicker [37, page 114], Oakley [65, Figures 1(b), 3(b)], Kornicker and Harrison-Nelson [43, Tables 8, 9], Harrison-Nelson and Kornicker [53, page 874].

Holotype. USNM 157988, ovigerous female.

Type Locality. Gulf of Mexico off Galveston, Texas, $28^{\circ} 10^{\prime} \mathrm{N}, 94^{\circ} 18^{\prime} \mathrm{W} ; 53.5 \mathrm{~m}$.

Distribution. Northwest Atlantic: Florida shelf, USA; predominantly from Gulf of Mexico from continental shelves of Florida, Alabama, Louisiana, and Texas.

Habitat. Benthic; from 6.1 to $148 \mathrm{~m}$.

Life History and Ontogeny. Female, male, from 1 to 4 eggs.

Comparisons. The infold of the caudal process of the left valve of $R$. gyre does not have the flat spines present on the left valve of $R$. darbyi. Rutiderma mortenseni Poulsen [10], is known only from a single adult male. The male of $R$. gyre differs from the male of $R$. mortenseni in having from 6 to 8 rather than from 10 to 12 bristles forming a row on the rostral infold (Kornicker [16, 62a]).

5.16. Rutiderma hartmanni Poulsen [10] (Figures 5(b)-5(c)). Rutiderma hartmanni Poulsen [10, pages 7, 17, 18, 22, 32-35, 41, 151, Table 1, Figure 8, Keys to species of Rutiderma (males), (females) pages 17-18], Kornicker [13, pages 70, 80, 678], Kornicker [54, page 43 (compares to R. leloeuffi)], Cohen and Kornicker [78, pages 21, 26], Kornicker and Cohen [80, page 740, Table 1], Kornicker and Meyers [14, page 4], Kornicker [81, page 127], Kornicker [63, ii, iii, pages 1-28, Figures 1-20], Kornicker [48, page 84], Kornicker [49, pages 136, 137, 140, Table 17], Kornicker [37, pages 114, 192], Kornicker and Harrison-Nelson [43, page 467, Tables 1, 7-9].

Not Rutiderma rostrata. Hartmann [82, pages 195, 196, 198,199 ] ( placed in synonymy of $R$. hartmanni by Poulsen [10]), Kornicker [81, page 127] (= Rutiderma pax Kornicker [81]), Rutiderma hartmanni. Cohen [20, pages 322, 324, 325, 331, 334 (possibly new subspecies “A”)].

Holotype. Female without eggs or embryos, Zoological Museum, University of Copenhagen.
Type Locality. SW Bay, San José Island, Pearl Islands, Gulf of Panama, East Pacific.

Distribution. Bay of Panama, Pacific Ocean.

Habitat. Depth $9 \mathrm{~m}$.

Comparisons. Rutiderma hartmanni differs from $R$. vox in that it has an upturned tip on the c-bristle (claw) of the second endopodial segment of the female mandible. Rutiderma hartmanni can be distinguished from $R$. kalkei by the c-bristle on the first antenna; it lacks filaments on $R$. hartmanni but has 1 on $R$. kalkei.

5.17. Rutiderma irrostrata Kornicker and Caraion [47] (Figure 5(d)). Rutiderma irrostratum Kornicker and Caraion [47, pages 3-6, 54, 56-60, 106, Table 1, Figures 2, 3, 49-51, Plate 31], Kornicker [30, Table 15], Kornicker [49, page 136], Kornicker and Harrison-Nelson [43, Tables 1, 8, 9].

Holotype. 291, female (A-1 stage), "Grigore Antipa" Museum of Natural History, Bucharest, Romania.

Type Locality. Station X053, $21^{\circ} 47^{\prime} 00^{\prime \prime} \mathrm{N}, 17^{\circ} 28^{\prime} 02^{\prime \prime} \mathrm{W}$, 260 m, Western Sahara.

Distribution. Northeast Atlantic off Western Sahara, Mauritania.

Habitat. Benthic; shelf-bathyal; from 94 to $250 \mathrm{~m}$; sand and mud.

Life History and Ontogeny. Ovigerous female, A-1 female and male, juvenile.

Stomach Contents. Harpacticoid copepod, polychaete, and a nematode in the gut.

Comparisons. The main tooth of the first exopodite segment of the fifth limb of females and advanced juveniles of previously described species consist of 3 or 4 large prongs and a proximal peg. The main tooth of the new species $R$. irrostrata bears only 1 large prong with 3 marginal teeth. The b-bristle of the seventh segment of the first antenna is minute, much smaller than that bristle on previously described species. The tip of the seventh limb of R. irrostrata is either bare or has a few minute spines, unlike the tip of the limb of other species which bears 2 opposing combs with well-developed teeth. In addition, the rostrum is totally lacking on $R$. irrostrata and in its place is a minute line. The degree of development of the rostrum of previously described species varies, but none is without a rostrum. The above differences may warrant future inclusion of this species in a new subgenus of Rutiderma (Kornicker and Caraion [47, page 60]).

5.18. Rutiderma judayi McKenzie [9] (Figures 5(e)-5(f)). Rutiderma judayi McKenzie [9, pages 58, 64, 66, Figures 3, Plate 1: Figure 2], Kornicker [13, page 678 (compares to $R$. species B)], Kornicker and Myers [14, pages 25-31, 34, (key to species of adult females of southern California, key to species of adult males of southern California), Figures 1518], Kornicker [48, page 84], Kornicker [37, page 114], Kornicker and Harrison-Nelson [43, Tables 8, 9], Frame et al. [45, Table 1, Figure 1 Fifth Row].

Holotype. USNM 110916, adult female.

Type Locality. Laguna Ojo de Liebre, Scammon Lagoon, Baja California, depth about $7 \mathrm{~m}$. 


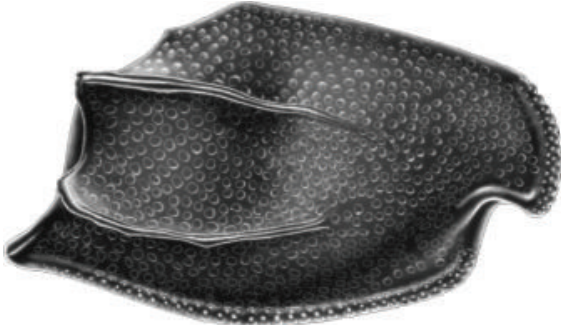

(a)

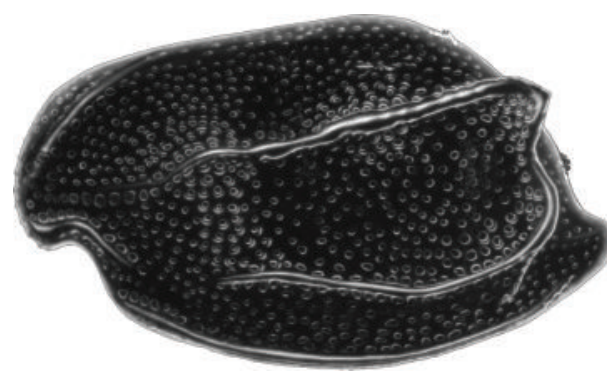

(c)

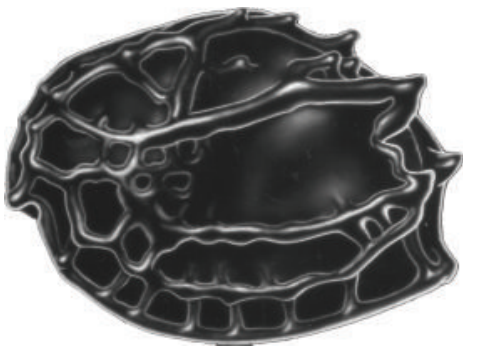

(e)

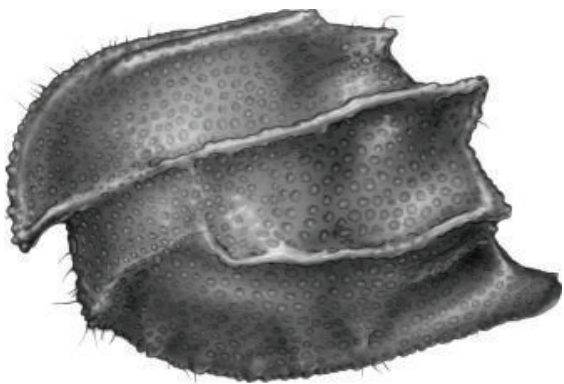

(g)

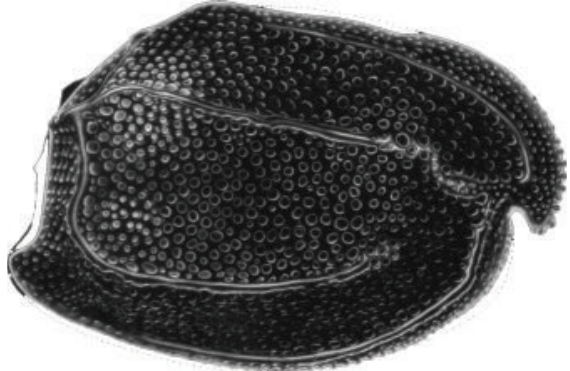

(b)

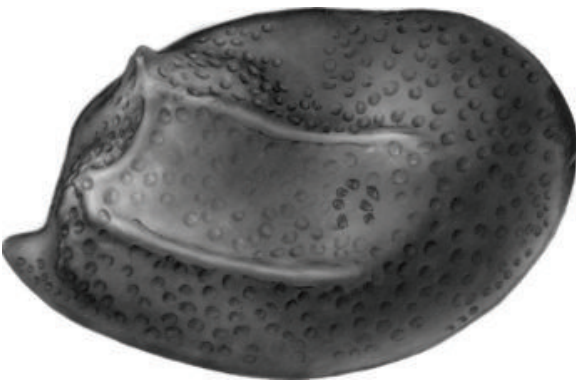

(d)

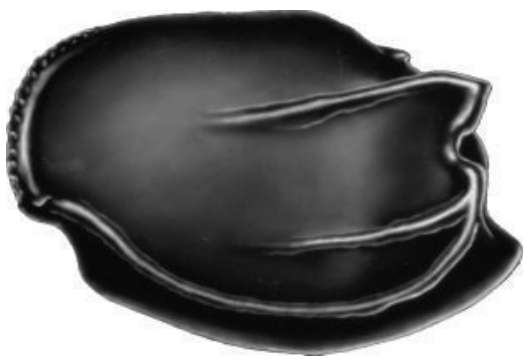

(f)

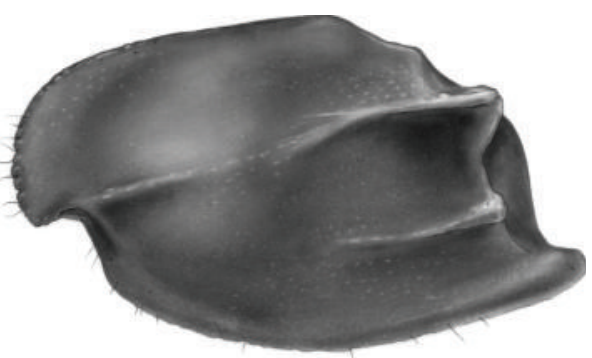

(h)

Figure 5: (a) Rutiderma gyre, male, $1.12 \mathrm{~mm}$. (b) R. hartmanni, female, $1.12 \mathrm{~mm}$. (c) R. hartmanni, male, $1.06 \mathrm{~mm}$. (d) R. irrostrata, female, $1.50 \mathrm{~mm}$. (e) R. judayi, female, $1.01 \mathrm{~mm}$. (f) R. judayi, male, $1.03 \mathrm{~mm}$. (g) R. kalkei, female, $1.08 \mathrm{~mm}$. (h) R. kalkei, male, $1.14 \mathrm{~mm}$.

Distribution. Northeast Pacific in Scammon Lagoon, Baja California, Mexico; San Diego and San Clemente Island, and La Jolla, California, USA

Habitat. Benthic, from 7 to $21 \mathrm{~m}$; fine to coarse sands, mostly poorly sorted, quartz and sometimes shells principle components, surface water temperature and salinity: from $18.5^{\circ}-25^{\circ} \mathrm{C}$, from $34-37 \%$; lagoon organic production rate: $50 \mathrm{mg} \mathrm{C} \mathrm{m}^{-3} \mathrm{~d}^{-1}$; part of lower lagoon faunal assemblage; $3 \mathrm{~cm}$ deep, eel grass in tide pools.

Life History and Ontogeny. Female, male.
Comparisons. Rutiderma judayi differs from R. vox in having a deep indentation at midheight of the posterior edge of the alar process on each valve. The carapace of $R$. juday $i$ is similar to $R$. apex but differs in that the female has a small process near the middle of the posterior margin that projects past the posterior end of the valve. The carapace is also similar to $R$. chessi but differs from it in that $R$. judayi has 3 instead of 2 main horizontal ribs, has more processes on the posterodorsal margin, and has a less acute caudal process. 
5.19. Rutiderma kalkei Kornicker [16] (Figures 5(g)-5(h)). Rutiderma kalkei Kornicker [16, pages 1, 10, 12, 15, 16, 25, 73-80, Table 1, Figures 3, 44-48], Kornicker [33, Table 3], Kornicker [48, page 84], Kornicker [37, page 114], Kornicker and Harrison-Nelson [43, Tables 8, 9], Harrison-Nelson and Kornicker [53, page 874].

Rutiderma kalke. Kornicker [16, page 12] (misspelling).

Holotype. USNM 159079, ovigerous female.

Type Locality. South Texas continental shelf, off Port Isabel, $26^{\circ} 10^{\prime} \mathrm{N}, 96^{\circ} 24^{\prime} \mathrm{W}, 91 \mathrm{~m}$.

Distribution. Gulf of Mexico: south Texas shelf, USA

Habitat. Benthic; $91 \mathrm{~m}$.

Life History and Ontogeny. Female, male, 3 eggs.

Stomach Contents. Nematode.

Comparisons. In lateral view the carapace of the new species R. kalkei resembles that of R. gyre, but the infold of the caudal process is quite different. The list on the caudal process of $R$. gyre bears an arc almost perpendicular to the ventral margin of the valve, whereas the middle of the arc on the caudal process of R. kalkei faces the ventral margin; the tip of the caudal process of $R$. kalkei is more broadly rounded than that of $R$. gyre (Kornicker [16, page 80]).

5.20. Rutiderma leloeuffi Kornicker [13] (Figure 6(a)). Rutiderma leloeuffi Kornicker [54, pages 2, 40-45, Figures 2932], Kornicker and Caraion [47, pages 2-4, 6, 54-56, 66, 103-105, Figure 48, Plates 28-30], Kornicker [30, Table 15], Kornicker [48, page 84], Kornicker [49, page 136], Kornicker and Harrison-Nelson [43, Tables 1, 8, 9].

Holotype. USNM 149330, adult female.

Type Locality. Station 18, near Grand Bassam, Ivory Coast, $5^{\circ} 12^{\prime} 05^{\prime \prime} \mathrm{N}, 3^{\circ} 49^{\prime} 05^{\prime \prime} \mathrm{W}, 20 \mathrm{~m}$.

Distribution. Northeast Atlantic: off Ivory Coast and Mauritania.

Habitat. Benthic; shelf-bathyal; from 20 to $150 \mathrm{~m}$; fine sand; water temperature and salinity: $28.9^{\circ} \mathrm{C}, 34.4 \%$.

Life History and Ontogeny. Female, A-1 male.

Comparisons. Rutiderma leloeuffi, new species, differs from $R$. hartmanni and $R$. mortenseni in having only 3 stout claws on the furca. It differs from $R$. normani in having a larger and more elongate carapace. The incisur is more distinct and the rostrum and caudal process more prominent on $R$. leloeuffi than on $R$. compressa, $R$. dinochelata, $R$. gerdhartmanni, and Rutiderma species A and B (Kornicker [54, page 43]).

5.21. Rutiderma licina Kornicker [16] (Figures 6(b)-6(c)). Rutiderma licinum Kornicker [16, pages 2, 4-8, 10, 12, 25, 47-54, 85, Table 1, Figures 2, 25-29, Plate 2], Kornicker [81, page 130, Figure 3c (compares with R. pax)], Kornicker [33, Table 3], Cohen and Morin [21, Figure 3 (compares copulatory organs with other families of Myodocopida)], Kornicker [48, page 84], Kornicker [37, page 114], Kornicker and Harrison-Nelson [43, Tables 8, 9], Oakley [65, Figures 1(b), 3(b)], Kornicker et al. [56, page 95], Harrison-Nelson and Kornicker [53, page 874].

Holotype. USNM 154186, ovigerous female.

Type Locality. Gulf of Mexico, off Galveston, Texas, $28^{\circ} 16^{\prime} \mathrm{N}, 94^{\circ} 06^{\prime} 30^{\prime \prime} \mathrm{W}, 53 \mathrm{~m}$.
Distribution. Northeast Atlantic: North Carolina continental shelf, USA: Gulf of Mexico: Florida, Alabama, and Texas continental shelves.

Habitat. Benthic; from 17 to $68 \mathrm{~m}$.

Life History and Ontogeny. Female, male, from 1 to 3 eggs.

Comparisons. All the following bear 3 strong claws on each lamella of the furca. The new species $R$. licina differs from $R$. tridens, $R$. mollita, and $R$. compressa, in that the female has a fairly deep incisur with overhanging rostrum, not a right angle forming the incisur as in the other three species (Kornicker [16, page 54]).

5.22. Rutiderma lomae Juday [58] (Figures 6(d)-6(e)) . Philomedes lomae Juday [58, pages 141, 142 (syntypes not extant)], Müller [46, pages 26,31], Skogsberg [23, page 380 (not in Philomedes)], Lucas [83, page 399 (mentions)], Poulsen [25, pages 358, 395 (compares to Scleroconcha)], McKenzie [9, page 57 (mentions)], Poulsen [10, pages 15-17, 22].

(?) Philomedes lomae. Hartmann [84, pages 230, 231, 247], Hartmann [85, pages 60, 61, 76, Table opposite pp. 83 (identification of specimens from El Salvador needs verification)], Hartmann-Schröder and Hartmann [86, pages 33, 34, 48], Hartmann [87, pages 171, 264 (identification of specimens from Chile needs verification)].

Rutiderma lomae. Kornicker and Myers [14, pages 1-2, Keys: 3-4 (Species of adult females from southern California, Species of adult males of southern California), pages 1018, 34, Figures 5-10], Kornicker [48, page 84], Kornicker [37, pages 114, 192], Kornicker and Harrison-Nelson [43, Tables 1, 8, 9].

Neotype. USNM 158258, adult female.

Neotype Locality. $33^{\circ} 34^{\prime} 15^{\prime \prime} \mathrm{N}, 118^{\circ} 00^{\prime} 45^{\prime \prime} \mathrm{W}$, California, USA (locality data for Juday [58], in Michael and McEwen $[88$, pages 133,143$])$.

Distribution. Off San Diego, off Santa Catalina Island, and Oxnard, California; (?) El Salvador; (?) Southeast Pacific: Bay of Caldera, Chile.

Habitat. Planktonic, surface [58]; benthic, sediment at $9.8^{\circ} \mathrm{C}$. (?) Benthic; upper sublittoral, shelly sand beach.

Life History and Ontogeny. Male, female, juvenile female, 4 eggs.

Comparisons. The males of $R$. lomae and $R$. apex are very similar except that the middle part of the vertical rib of the carapace of $R$. apex seems to be better developed. The females of $R$. lomae and $R$. apex can be differentiated by the vertical rib at the posterior end of the alar process on the carapace; on $R$. lomae, there is a space near the middle, but, on R. apex, it is continuous. Rutiderma lomae females have a shorter caudal process, more bristles on the list of the caudal process, and a longer b-bristle on the first antenna than female specimens of $R$. vox. R. vox also has a stouter fourth claw on the furca, and it lacks a minute bristle near the middle of the margin.

5.23. Rutiderma mollita Darby [74] (Figures 6(f)-6(g)). Rutiderma mollita Darby [74, pages 29, 58. Plate 15: Figures 1, 2], Kornicker [13, page 74], Kornicker and Caraion 


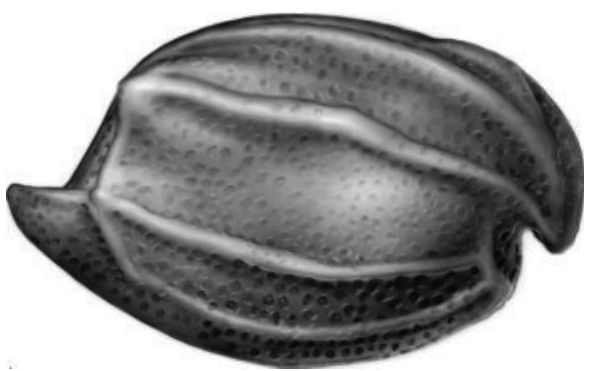

(a)

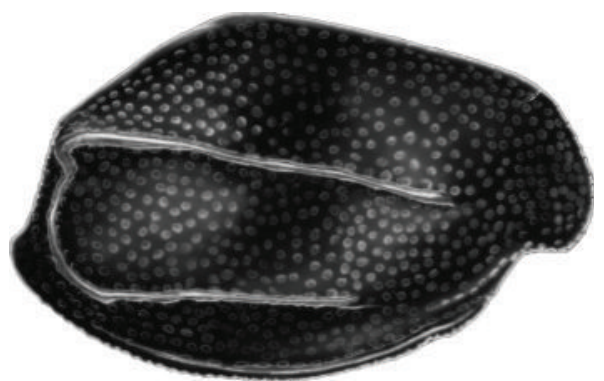

(c)

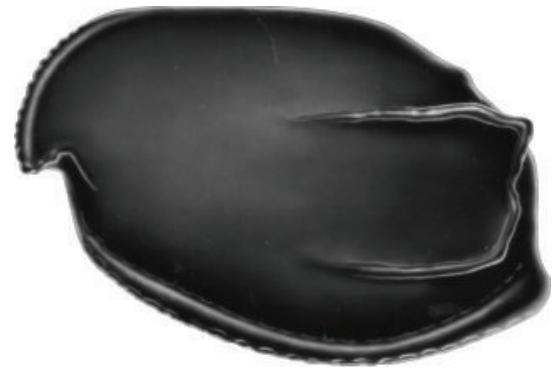

(e)

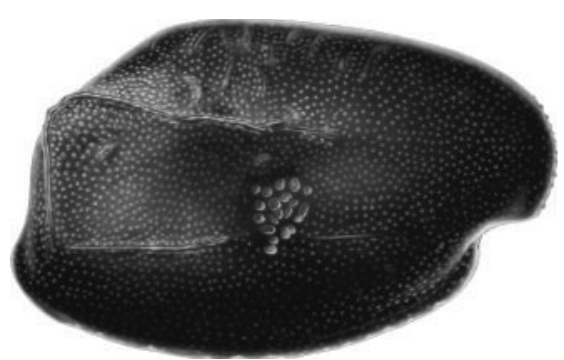

(g)

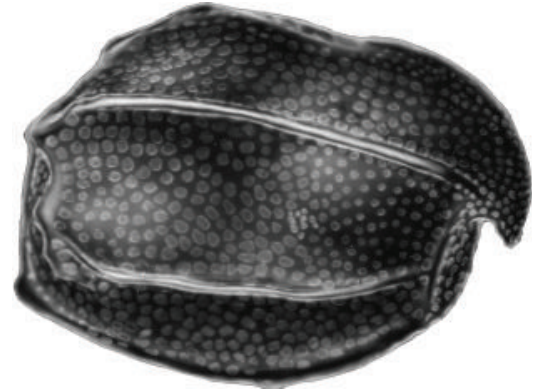

(b)

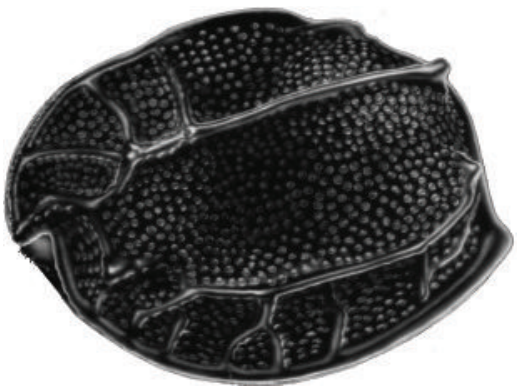

(d)

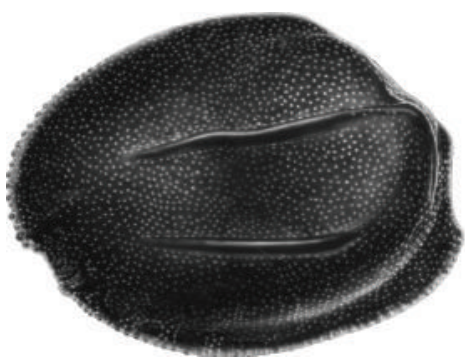

(f)

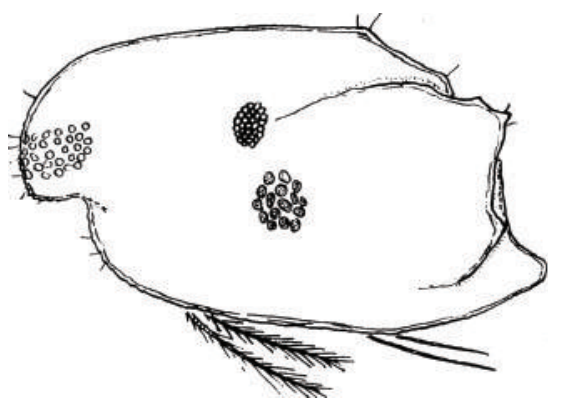

(h)

Figure 6: (a) Rutiderma leloeuffi, female or instar, 1.99 mm. (b) R. licina, female, $1.02 \mathrm{~mm}$. (c) R. licina, male, $1.08 \mathrm{~mm}$. (d) R. lomae, female, $1.46 \mathrm{~mm}$. (e) R. lomae, male, $1.21 \mathrm{~mm}$. (f) R. mollitum, female, $1.46 \mathrm{~mm}$. (g) R. mollitum, male, $1.47 \mathrm{~mm}$. (h) R. mortenseni, male, $1.07 \mathrm{~mm}$.

[47, page 66 (compares to R. tridens)], Oakley [65, Figures 1, 3 (compares to other species)].

Rutiderma mollitum. Kornicker [16, pages 2-7, 12, 13, 15, 25, 28-36, 54, 85, Table 1, Figures 3, 12-17], Kornicker [33, Table 3], Kornicker [37, page 114], Grabe [18, Tables 2, 5], Kornicker and Harrison-Nelson [43, Tables 8, 9], Oakley
[65, Figures 1(b), 3(b)], Harrison-Nelson and Kornicker [53, page 874].

Holotype. UMMP 48791, female, University of Michigan, Museum of Paleontology.

Type Locality. Continental shelf off Sapelo Island, Georgia, from $19.8 \mathrm{~m}$. 
Distribution. Northwest Atlantic: shelf off South Carolina to Florida; Gulf of Mexico: Tampa Bay, shelf off Florida, Alabama.

Habitat. Benthic; from 5.4 to $190 \mathrm{~m}$; salinity and temperature: about $30 \%$ (Darby) and $35 \%, 32^{\circ} \mathrm{C}$; fine to coarse sand.

Life History and Ontogeny. Female, male, 3 to 4 eggs.

Remarks. The species differs from other species in the genus in having the lamellar prolongation of the selvage continuing along the anterior ridge of the caudal process. In other species the selvage and lamellar prolongation are present along the outer edge of the caudal process (Kornicker [16, page 36]).

5.24. Rutiderma mortenseni Poulsen [10] (Figure 6(h)). Rutiderma mortenseni Poulsen [10, pages 7, 8, 11, 14, 17, 38-41, 43, 44, Figure 11], Kornicker [13, pages 70, 80], Kornicker [54, page 43], Cohen and Kornicker [78, pages 21, 26], Kornicker and Cohen [80, Table 1], Kornicker [16, pages 12, 13, 25, 28, 62, 67, 70, Table 1], Kornicker [33, Table 3], Kornicker and Harrison-Nelson [43, Tables 8, 9], Kornicker et al. [56, page 95].

Holotype. Male, Zoological Museum, University of Copenhagen.

Type Locality. Virgin Islands, West Indies.

Distribution. Type locality only.

Life History and Ontogeny. Male.

Comparisons. Rutiderma mortenseni has 10-12 bristles in the rostral infold, whereas $R$. gyre has just 6-8. On $R$. arcuatilis, the curvature of the list of the caudal process has a low angle with the ventral margin, but, on $R$. mortenseni, it forms a $45^{\circ}$ angle. The two species also differ in that $R$. arcuatilis has bristles all-on the ends.

5.25. Rutiderma normani Poulsen [10] (Figures 7(a)-7(b)). Rutiderma normani Poulsen [10, pages 7, 8, 11, 14, 17, 18, 2232, 34, 38, 41, 452, Figures 4-7, 150], Cohen and Kornicker [78, pages 21, 26], Hartmann [28, Figure 389b] (according to Hartmann [60] and Poulsen [10]), Kornicker [13, pages 70, 74, 80], Kornicker [54, page 43], Hanai et al. [89, page 49], Kornicker and Cohen [80, Table 1], Hiruta and Maddocks [90, page 674, Table 1], Jones [91, Table 1], Kornicker [63, page 14], Kornicker [48, page 84], Kornicker [49, pages 136, 140, Table 17], Kornicker [37, page 114], Kornicker and Harrison-Nelson [43, Tables 1, 7-9]. hagen.

Holotype. Zoological Museum, University of Copen-

Type Locality. Koh Kahdat, Thailand, 8-10 m.

Distribution. Type locality and Koh Mesan-Cape Liant, Koh Kam, and Koh Chung on the south coast of Thailand.

Habitat. Benthic; from 2 to $17 \mathrm{~m}$; sand, gravel, and corals.

Life History and Ontogeny. Female, ovigerous female, male, juvenile male, 1 juvenile, 4 eggs.

Comparisons. Rutiderma $d u x$ is close to $R$. normani Poulsen [10, page 22], and they could be conspecific. The female sixth limbs of the two species differ in that the ventral margin of the end segment of $R$. normani is straight
(Poulsen [10, Figure 4j]), whereas the anterior 3 bristles on the end segment of $R$. dux are on a long projection. Also, Poulsen [10, pages 26, 28] described both the female and male furcae of $R$. normani as having 3 main claws and 3 secondary claws, whereas the female and male furcae of $R$. dux have 4 main claws and 2 secondary claws. Rutiderma normani has a shorter and less elongate carapace than $R$. leloeuffi. $R$. normani and $R$. arx differ in that $R$. arx lacks lateral ribs on the carapace, whereas $R$. normani does have lateral ribs. While $R$. normani has a serrate list on the infold of the caudal process of the right valve, $R$. vox does not. According to Poulsen [10], the males of $R$. rostrata and $R$. normani are similar but $R$. normani has just 1 bristle on the ventral margin of the mandible basale and has at least 7 bristles on the end segment of the sixth limb. The shell height of $R$. rostrata is also greater than that of $R$. normani (Poulsen $[10])$. The females of $R$. normani and $R$. rostrata differ in that $R$. rostrata does not have the prominent longitudinal shellridges that $R$. normani does (Poulsen [10]).

Remarks. Poulsen [25, Figure 7] identified two juveniles of Rutiderma normani as "females?", but on page 30 he stated for the same two specimens: "As the copulatory limbs and the secondary sexual characters of the first and second antennae were not developed, the sex of these two young larvae could not be ascertained." The endopod of the second antenna of the adult female of the species has only one short segment (Poulsen [10, page 23, Figures 4f. $\left.f^{\prime}\right]$ ), whereas, the endopodes of the second antennae of the two juveniles (Figures 7b, 7i) are elongate. Therefore, it is the opinion of Kornicker (herein) that the two juveniles are males.

5.26. Rutiderma oakley Kornicker, new species (Figures 7(c)7(d)). Etymology. The species is named after Todd H. Oakley, University of California.

Rutiderma species B Kornicker [16, pages 10, 80-85, Table 1, Figures 49-51], Cohen [20, pages 322, 324, 325, 331, 333], Kornicker [37, page 114], Kornicker and HarrisonNelson [43, Tables 8, 9].

Holotype. USNM 159019, ovigerous female.

Type Locality. Gulf of Mexico, continental shelf off South Texas, USA, Transect 4, 25 may $1977,26^{\circ} 10^{\prime} \mathrm{N}, 97^{\circ} 08^{\prime} \mathrm{W}$, depth $15 \mathrm{~m}$. 80].

Paratypes. Rutiderma species B in Kornicker [16, page

Distribution. Belize, vicinity of Carrie Bow Key, $16^{\circ} 49^{\prime} \mathrm{N}$, $88^{\circ} .05^{\prime} \mathrm{W}$, Continental shelf off South Texas, $26^{\circ} 10^{\prime} \mathrm{N}$, $97^{\circ} 08^{\prime} \mathrm{W}$, depth ca. $15 \mathrm{~m}$.

Description. A detailed description of adult male and female is presented in Kornicker [16, pages 80-85, Figures 49-51]. The furca bears 4 stout claws followed by 2 small claws on each lamella. Tip of terminal claw of second endopodial segment of adult female mandible with elongate slightly upturned tip.

Comparisons. The new species Rutiderma oakley differs from $R$. mollita in having 4 main claws on each lamella of the furca. It differs from $R$. darbyi in not having serrate list on the caudal process of the left valve. Rutiderma oakley differs 


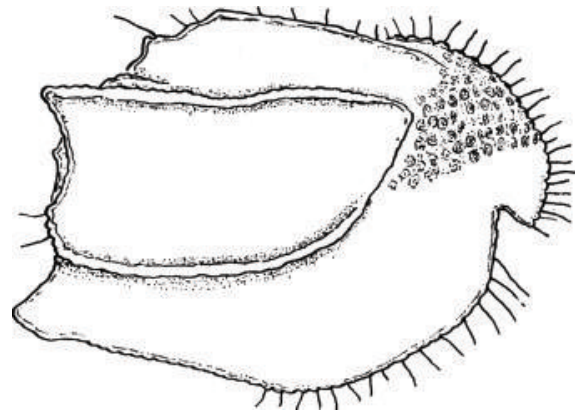

(a)

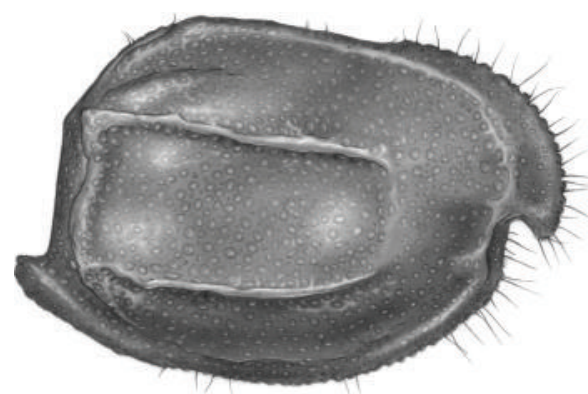

(c)

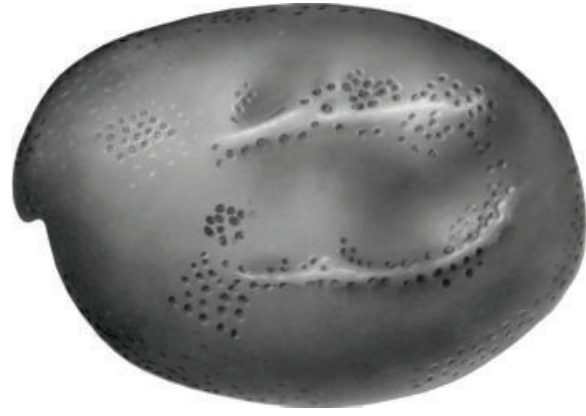

(e)

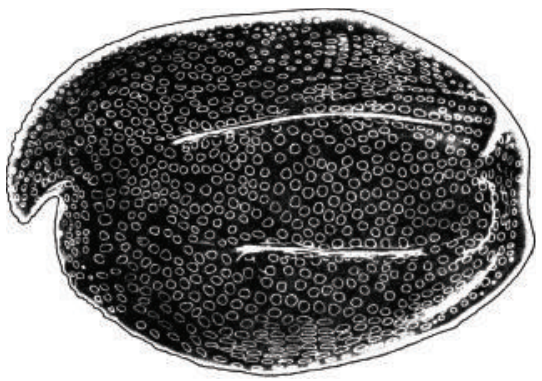

(g)

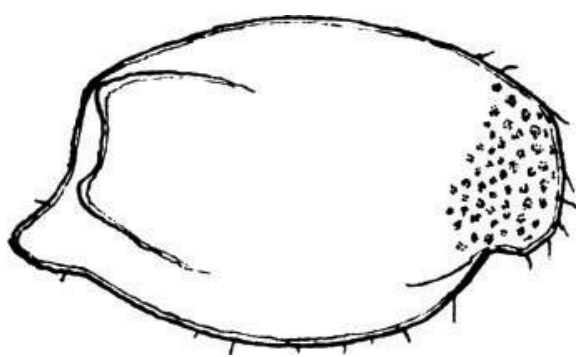

(b)

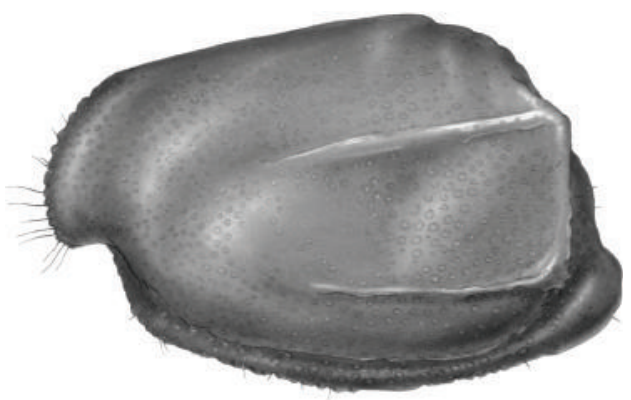

(d)

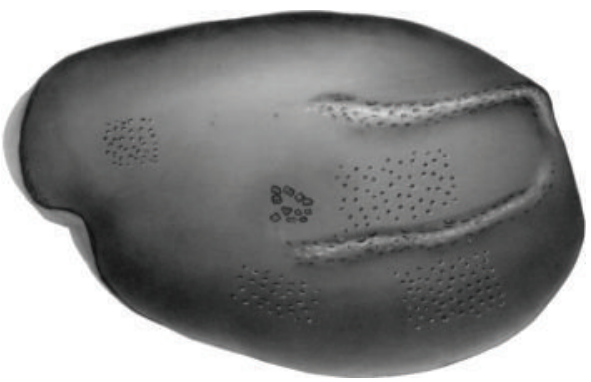

(f)

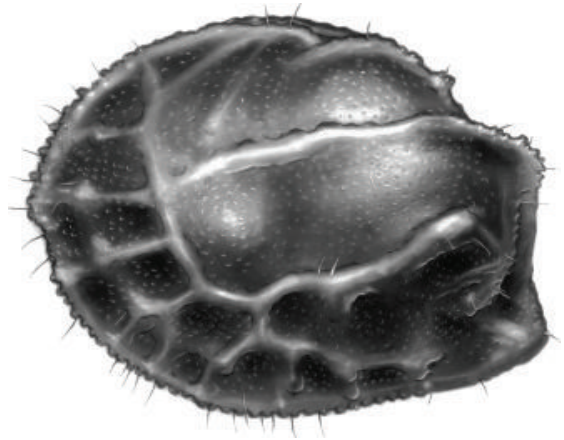

(h)

Figure 7: (a) Rutiderma normani, ovigerous female, $1.25 \mathrm{~mm}$. (b) R. normani, male, $1.2 \mathrm{~mm}$. (c) R. oakley, female, $1.11 \mathrm{~mm}$. (d) R. oakley, male, $0.98 \mathrm{~mm}$. (e) R. ovata, female, $1.52 \mathrm{~mm}$. F. R. ovata, male, $1.59 \mathrm{~mm}$. (g) R. pax, female, $1.00 \mathrm{~mm}$. (h) R. rex, ovigerous female, $0.92 \mathrm{~mm}$.

from $R$. cohenae in that the caudal process of the left valve is broadly rounded rather than triangular. Only $R$. cohenae and $R$. oakley have a prolonged tip at the end of the terminal claw of the second endopodial segment of the adult female mandible and on juveniles in both sexes. Most species of Rutiderma do not have a prolonged tip on the terminal claw on the second segment.
5.27. Rutiderma ovata Kornicker [13, 54] (Figures 7(e)-7(f)). Rutiderma ovata Kornicker [13, pages 10, 29, 47, 60, 65, 70, 74, 80, 646, key: 647, 650, 657-673, Figures 407, 411, 412-424, 432 h-k], Kornicker [30, Table 15], Kornicker and Cohen [80, Table 1], Kornicker [63, pages 25, 26], Kor-nicker [37, page 114], Kornicker and Harrison-Nelson $[43$, Tables 8,9$]$. 
Holotype. USNM 137683, gravid female.

Type Locality. "Eltanin Cruise 3, station 71, sample 71-26, $31^{\circ} 05^{\prime} \mathrm{S}, 71^{\circ} 44^{\prime} \mathrm{W}$ to $31^{\circ} 06^{\prime} \mathrm{S}, 71^{\circ} 47^{\prime} \mathrm{W}$, depth $192-176 \mathrm{~m}$, shelf west of Chile."

Distribution. Southeast Pacific: off Chile: $31^{\circ} 10^{\prime} \mathrm{S}$, $71^{\circ} 56^{\prime} \mathrm{W} ; 31^{\circ} 05^{\prime} \mathrm{S}, 71^{\circ} 44^{\prime} \mathrm{W} ; 31^{\circ} 06^{\prime} \mathrm{S}, 71^{\circ} 47^{\prime} \mathrm{W}$.

Habitat. Benthic; from 192 to $176 \mathrm{~m}$ (shelf); (?) $1834 \mathrm{~m}$ (probably not collected at that depth).

Life History and Ontogeny. Female, male, A-1 juvenile male, juvenile, from 2 to 4 eggs.

Stomach Contents. Nematodes, crustacean fragments, annelid spines, copepods, and sedimentary particles.

Comparisons. Because of the absence of a projecting posteroventral caudal process, the carapace of the new species resembles that of $R$. rotunda Poulsen [10]. The incisur of $R$. ovata is much more developed than that of $R$. rotunda, also the latter has 6 proximal bristles on the seventh limb compared vto just 4 on $R$. ovata. The furca of $R$. ovata bears 6 claws compared to 5 for $R$. rotunda. Rutiderma californica McKenzie [9], which is synonym of $R$. rotunda, also has 6 proximal bristles on the seventh limb and the carapace is more ornate than that of $R$. ovata.

In addition to difference in shape of the carapace, $R$. rostrata Juday [58], differs from $R$. ovata in having 4 strong claws on the furca. Rutiderma compressa Brady and Norman [55] has a carapace with a projecting posteroventral caudal process, which is absent on R. ovata (Kornicker $[13$, page 673]).

5.28. Rutiderma pax Kornicker [81] (Figure 7(g)). Rutiderma rostrata Hartmann [82, pages 195, 196, 198, 199], Kornicker [30, page 4] (note in synonymy that species not $R$. rostratum Juday [58]).

Rutiderma rostrata. Hartmann [82, page 195 (misspelling)].

Rutiderma hartmanni. Poulsen [10, page 32 (part; includes only $R$. rostrata of Hartmann in synonymy)].

Rutiderma pax. Kornicker [81, pages 127-131, Figures l3a], Hartmann and Petersen [35, page 158].

Holotype. K-27314 (G1), ovigerous female, Zoologisches Museum Hamburg.

Type Locality. Off Mejanguera Island, Gulf of Fonseca, El Salvador, sublittoral, 12 m (Hartmann [82, pages 195, 196]).

Distribution. Northeast Pacific: El Salvador: Mejanguera.

Habitat. Benthic; 12 m; mud.

Life History and Ontogeny. Female, 1 egg.

Comparisons. Three species similar to $R$. pax in having carapaces with poorly defined lateral ribs and in not having a prominent backward projecting caudal process are $R$. rostrata Juday [58], R. licina Kornicker [16], and a species identified as $R$. rostrata Juday by McKenzie [9], but which is probably a new species (USNM 110918). The three species can be distinguished from $R$. pax, as well as from each other, by the morphology of the tip of the stout terminal claw of the second endopodial segment of the female mandible (Kornicker [81, pages 130-131]).
5.29. Rutiderma rex Kornicker [49] (Figure 7(h)). Rutiderma rex Kornicker [49, pages 140-144, Tables 1, 2, Figures 8183], Kornicker and Harrison-Nelson [43, Tables 8, 9].

Holotype. MNHN Os 277, ovigerous female, Muséum National d'Histoire Naturelle, Paris, France.

Type Locality. "Station 101-DS, 8 Apr 1977, NW Île du Lys, Glorioso Islands, $11^{\circ} 25^{\prime} 42^{\prime \prime} \mathrm{S}, 47^{\circ} 19^{\prime} 30 \mathrm{E}, 26 \mathrm{~m}$.”

Distribution. Collected only at type-locality, from sediment. (Females only).

Life History and Ontogeny. Adult female, ovigerous females.

Comparisons. The carapace of $R$. rex with its rugosity and minute incisur resembles that of $R$. compressa Brady and Norman [55, page 673]. The carapace of $R$. rex is much smaller; the tip of the claw-like c-bristle on the second endopodial segment of the mandible of $R$. compressa is produced and pointed (Brady and Norman [55], Plate LVIII: Figure 14), unlike that of $R$. rex. Only the male is known of Rutiderma fusca (Poulsen [10, page 41]), so its carapace and most appendages are not directly comparable with those of the female $R$. rex (male unknown). However, $R$. fusca has a pointed Bellonci organ (Poulsen [10, Figure 1]), whereas that of $R$. rex is broadly rounded, indicating that they are not conspecific. The small size and rugosity of the carapace of $R$. rex easily distinguish it from $R$. arx, the only other species of Rutiderma in the present collection; they differ also in the morphology of the Y-sclerite, which is unbranched in $R$. arx and branched in $R$. rex. The carapace of $R$. rex differs from that of $R$. vox Kornicker [48, page 78 ] in having a smaller rostral projection (Kornicker [49, page 144]).

5.30. Rutiderma rostrata Juday [58] (Figures 8(a)-8(b)). Rutiderma rostrata Juday [58, pages 142, 147-149, Plate 20: Figures 8-13], Müller [46, page 35].

Rutiderma (Rutiderma) rostrata. Kornicker [6, pages 236, 237].

(?) Rutiderma rostrata. Lie [11, pages 274, 288, 550 (specimens not available; identification needs verification)], Lie and Kisker [12, page 2279].

Rutiderma rostratum. Kornicker [13, pages 70, 80, 678], Kornicker and Myers [14, pages 1-10, keys: 3-4 (adult females; adult males), Figures 1-4], Cohen and Kornicker [78, pages 21, 26], Kornicker and Cohen [80, Table 1], Kornicker [81, pages 127-131, Figures 3b,d (compares to $R$. pax)], Kornicker [48, page 84], Kornicker [37, page 114], Kornicker and Harrison-Nelson [43, Tables 8, 9].

Not Rutiderma rostrata. Hartmann [82, pages 195, 196, 198, 199] (= R. pax Kornicker [16]), Hartmann [60, page 328], Poulsen [10, pages 6-9, 11, 14, keys: 17-18, 18-22, 3135 , 38, 41, 43, Figures 2, 3, Table 1 (=R. species)], McKenzie [9, pages 58, 62-64, Figure 2; Plate 1: Figure 3, (R. species)].

Holotype. Female, unique specimen, not extant.

Type Locality. Glorietta Bight, San Diego Bay.

Distribution. Northeast Pacific: off California, USA: San Diego, Oxnard, Monticeito. (?) Northeast Pacific: Washington, USA: Puget Sound and off northern coast about $125^{\circ} \mathrm{W}$ between $47^{\circ} \mathrm{N}$ and $48^{\circ} \mathrm{N}$. 


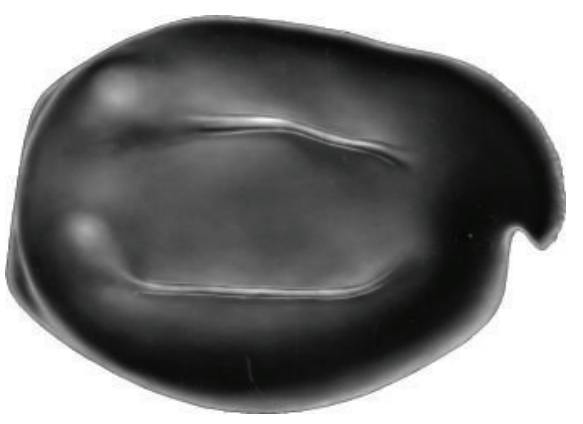

(a)

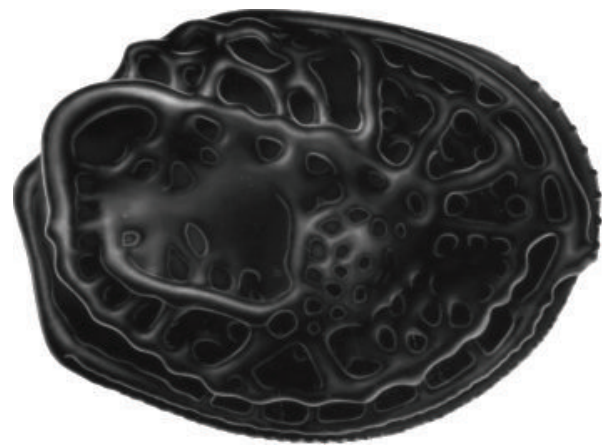

(c)

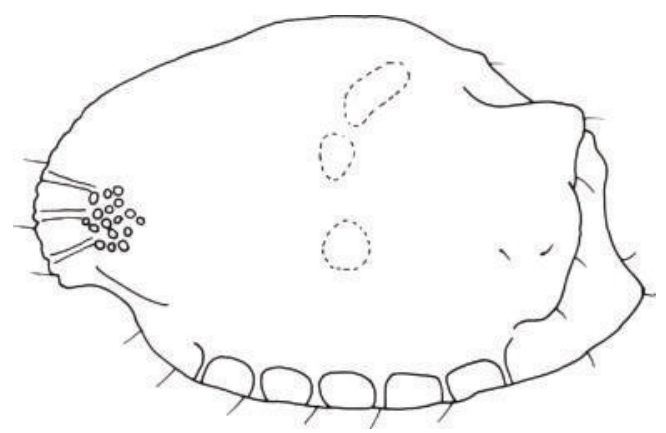

(e)

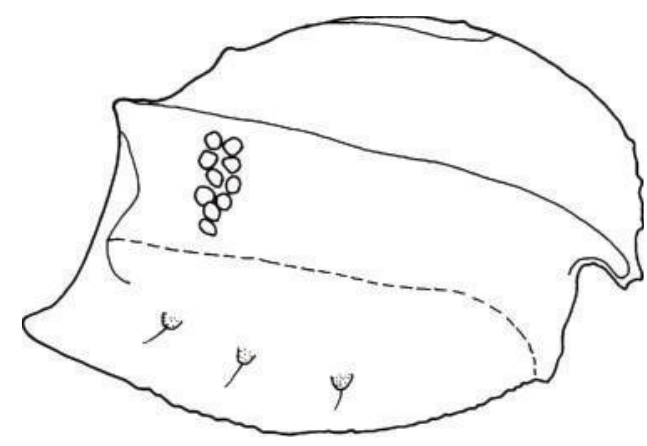

(g)

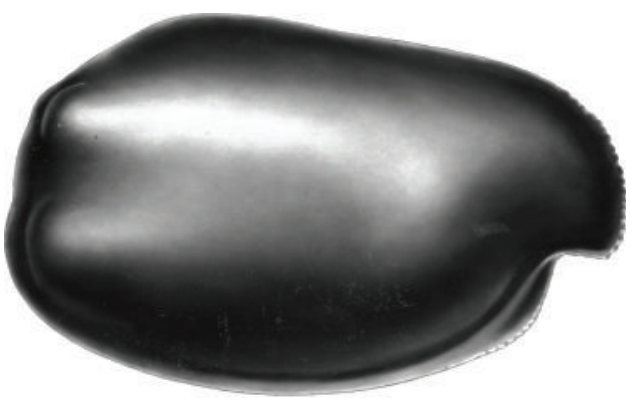

(b)

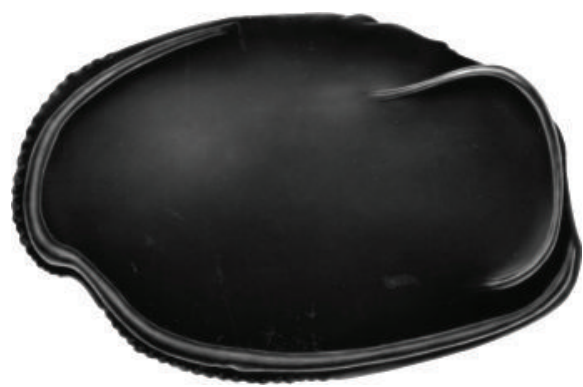

(d)

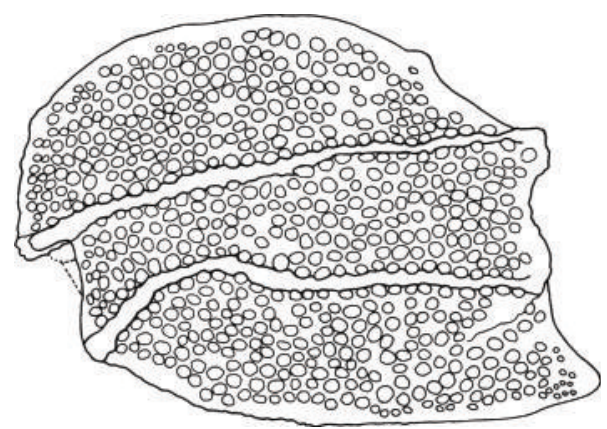

(f)

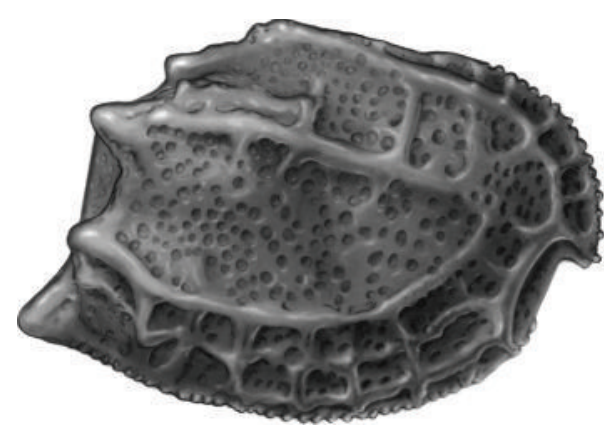

(h)

Figure 8: (a) Rutiderma rostrata, female, $1.32 \mathrm{~mm}$. (b) $R$. rostrata, male, $1.29 \mathrm{~mm}$. (c) $R$. rotunda, female, $1.33 \mathrm{~mm}$. (d) $R$. rotunda, male, $1.24 \mathrm{~mm}$. (e) R. sagax, male, $1.05 \mathrm{~mm}$. (f) R. schroederi, female, $1.73 \mathrm{~mm}$. (g) R. schroederi, instar III, $1.09 \mathrm{~mm}$. (h) R. sterreri, female, $1.10 \mathrm{~mm}$. 
Habitat. Benthic: from 11 to $22 \mathrm{~m}$; sand. (?) Benthic; from 15 to $317 \mathrm{~m}$; sand, sand and mud, mud, mud and gravel, fine sand mixed with shell fragments.

Life History and Ontogeny. Female, male, 2 eggs.

Comparisons. $R$. rostrata is similar to $R$. pax in having carapaces with poorly defined lateral ribs and in not having a prominent backward projecting caudal process. Rutiderma hartmanni differs from $R$. rostrata in that the shell of $R$. rostrata lacks riblets or ridges and is ovular, whereas, in $R$. hartmanni, the shell is ridged and has a posterior pointing process (Poulsen [10]).

5.31. Rutiderma rotunda Poulsen [10] (Figures 8(c)-8(d)). Rutiderma rotunda Poulsen [10, pages 7,17 , key to females: 17-18, 22, 34-38, 43, 455, Table 1, Figures 9, 10 (Holotype: Female, Zoological Museum, University of Copenhagen, Denmark; type-locality: "La Jolla, California, shallow water")], Cohen and Kornicker [78, pages 21, 26], Kornicker [13, pages 70, 74, 80, 673], Kornicker [63, page 14].

Rutiderma californica. McKenzie [9, pages 58, 65, 66, Figure 4; Plate 1: Figure 1], Kornicker and Meyers [14, page 18 (Holotype: Female, USNM 110911; type locality: "Laguna Ojo de Liebre. ..Scammon Lagoon, Baja California, Mexico")], Kornicker [13, page 673 (compares to R. ovata)].

Rutiderma rotundum. Kornicker and Myers [14, page 2], emendation, Key to species of adult females and males of southern California pages 3-4, 18-25, Figures 11-14, Kornicker [37, page 114], Kornicker and Harrison-Nelson [43, Tables 1, 8, 9], Frame et al. [45, pages 337, 339, Table 1, Figure 1 Fifth row].

Distribution. Northeast Pacific: California, USA: off La Jolla. (Poulsen), Irvine Beach, Ocean Beach (San Diego), between Newport Beach and Laguna Beach; Mexico: Scammon Lagoon, Baja, California.

Habitat. Benthic; from 0.3 to $<22 \mathrm{~m}$; shallow water, rocks with algae, eel grass, tide pools; fine to coarse sand, mostly poorly sorted, mainly quartz or shell, $18.5^{\circ} \mathrm{C}$ surface salinity from 34-37\%o, productivity about $50 \mathrm{mg} \mathrm{C} \mathrm{m}^{-3} \mathrm{~d}^{-1}$; eel grass and tide pool vegetation.

Life History and Ontogeny. Females, male, juvenile females, from 2 to 4 eggs.

Comparisons. The carapace of $R$. rotunda is similar to $R$. ovata because of the absence of a projecting posteroventral caudal process. It differs from $R$. ovata in that it has a less well-developed incisur and a more ornate carapace. Rutiderma rotunda also differs from $R$. compressa in the incisur. Rutiderma compressa has a right angle between the incisur and the margin, whereas $R$. rotunda has an obtuse angle.

5.32. Rutiderma sagax Kornicker [50] (Figure 8(e)). Rutiderma sagax Kornicker [50, pages 28-31, Figures 17-18, Table 1, Appendices 1-2].

Holotype. AM P45365, male.

Type Locality. Darwin, Australia, station JLB Darwin 302 and 305 combined (Station 302: Channel Island, 20 August 1982, mud; station 305 (same as station 304): East Point, 22 Aug 1982), both samples from intertidal washings of algae and substrate.
Distribution. Collected only at type locality.

Habitat. Mud.

Life History and Ontogeny. Adult male.

Comparisons. This species differs from the previously described species of the genus in having 7 furcal claws (3 primary and 4 secondary). The carapace of $R$. sagax is readily separated from that of $R$. $d u x$ because of the absence of flat pointed spines on the infold of the caudal process of the left valve. In addition to having fewer claws, the furca of $R$. dux differs from that of $R$. sagax in having 4 rather than 3 primary claws (Kornicker [50, page 31]).

5.33. Rutiderma schroederi, Kornicker and Iliffe [77] (Figures $8(f)-8(g))$. Rutiderma schroederi, Kornicker and Iliffe [77, pages 53-61, Figures 33-38, Tables 1, 2, Appendix], Kornicker [42, pages 798, 808-810, Figure 7], Kornicker and Harrison-Nelson [43, Tables 7-9], Kornicker et al. [56, page 95, Table 1].

Holotype. USNM 194472, adult female.

Type Locality. Station 94-018, transect AA Buoy, Exuma Sound, depth $67 \mathrm{~m}$.

Distribution. Bahamas: Great Exuma Island, Lee Stocking Island, depth 67-100 m.

Habitat. Sandy sediments on ledges of upper slope and submarine escarpment.

Life History and Ontogeny. Female with small, unextruded eggs, instar III, late instar.

Stomach Contents. Crustacean fragments.

Comparisons. The carapace of $R$. schroederi outwardly resembles that of $R$. darbyi Kornicker [16, page 36]. The infold of the caudal process of the left valve of $R$. schroederi is without the pleated ruffle present on $R$. darbyi (the ruffle is easily visible through the outside of the left valve). The carapace of $R$. schroederi also outwardly resembles $R$. gyre Kornicker [16, page 54], but the infold of the caudal process of $R$. schroederi is without the vertical crescent list present on $R$. gyre. The endopodites of the second antennae of $R$. darbyi and $R$. gyre are without the ringed posterior bristle present on $R$. schroederi. The carapace of $R$. schroederi also outwardly resembles that of $R$. cohenae Kornicker [16, page 62], which was collected near San Salvador, Bahamas, and Key West, Florida, from subtidal to $4 \mathrm{~m}$ depth (Kornicker [16, page $62]$ ). The morphology of the infolds of the caudal processes of the two species is also similar. The rostral infold of $R$. schroederi bears 17 bristles compared to 7 or 8 for $R$. cohenae. The length of the carapace of the unique female of $R$. schroederi is $1.73 \mathrm{~mm}$, compared to a range of 1.24 to $1.29 \mathrm{~mm}$ for two females of $R$. cohenae (Kornicker [16, page 63]). A major difference between the two species occurs in the mandible: the c-bristle of the second endopodial segment of $R$. cohenae has a prolonged finger-like tip that is absent on $R$. schroederi. Slides of three type-specimens of $R$. cohenae were reexamined during the present study, and all 6 limbs have the long finger-like tip on the c-bristle. The two specimens of $R$. schroederi examined do not have the long finger-like tip, but the tip of one limb of the holotype is obviously broken. The endopodites of the second antennae of $R$. schroederi and $R$. cohenae both share the unusual character of having a fairly long posterior bristle; the bristle is ringed in $R$. schroederi 
and unringed in $R$. cohenae, but more specimens should be examined to determine whether this character might be variable (Kornicker and Iliffe [77, page 61]).

Internal muscles are of limited use in identifying segments of $R$. schroederi. The interpretation of segmentation of the female fifth limb of this species is based mainly on that derived from the study of the fifth limb of Isocypridina (Kornicker [41, 42, pages 800, 806, 808]).

5.34. Rutiderma sterreri Kornicker [30, 31] (Figures 8(h), 9(a)). Rutiderma sterreri Kornicker [31, pages 1, 2, 58, Figures 3-5], Kornicker [16, pages 11-13, 25, 70-73, Figures 41-43], Kornicker [33, Table 3], Maddocks et al. [34, page 282, Figure 90 (part)], Kornicker [37, page 114], Kornicker and Iliffe [71, pages 43, 45, Figures 25, 26, Tables 4-8, Appendix], Kornicker [48, page 84], Kornicker and Harrison-Nelson [43, Tables 8, 9].

Holotype. USNM 158115, ovigerous female.

Type Locality. Station 630820, Harrington Sound, Bermuda, about $11 \mathrm{~m}$.

Distribution. Northwest Atlantic: Bermuda.

Habitat. Benthic; from intertidal to $11 \mathrm{~m}$; algae, grasses, oysters, shells, mud with grass, mud, coarse sand with Thalassia and Halimeda overlying mud, red sponge and algae, sand and mud under rocks.

Life History and Ontogeny. Female, male, 4 eggs. 8]).

Parasites. Choniostomatid copepod (Kornicker [31, page

Comparisons. The species $R$. sterreri differs from $R$. dinochelata in that the tip of the Bellonci organ is rounded, not pointed. The lengths of the three specimens of $R$. dinochelata listed by Kornicker [6, page 237] ranged from 1.14 to $1.22 \mathrm{~mm}$ (average $1.18 \mathrm{~mm}$ ). The lengths of seven ovigerous females of $R$. sterreri measured herein ranged from 0.94 to $1.10 \mathrm{~mm}$ (average $1.00 \mathrm{~mm}$ ). A closer comparison of the two species will require a better description of $R$. dinochelata (Kornicker [31, page 8]).

5.35. Rutiderma tridens Kornicker and Caraion [47] (Figures 9(b)-9(c)). Rutiderma tridens Kornicker and Caraion [47, pages 3-5, 54, 60-66, Figures 1-3, 52-55, Plates 32, 33], Kornicker [30, Table 15], Kornicker [49, page 136], Kornicker [37, page 114], Kornicker [48, page 84], Kornicker and Harrison-Nelson [43, Tables 1, 8].

(?) Rutiderma compressa. Müller [59, pages 53, 92, 93, Plate 7: Figures 1-13]. (questionably referred to $R$. tridens by Kornicker and Caraion [47, pages 60,65]: "The carapace of $R$. tridens resembles that of the female of $R$. compressa from South Africa illustrated by Müller [59, Plate 7:1]. Müller's specimen has been put into synonymy of $R$. tridens with a question, because the first antenna illustrated by Müller [59, Plate 7:2] does not show a lateral bristle on the second segment, but this could be because the illustration is a medial view of the limb. The first, third, and fourth endites of the sixth limb illustrated by Müller [59, Plate 7:12] have a different number of bristles than do the same endites of the two specimens of $R$. tridens described herein, but this could be the result of intraspecific variability."), Müller [46, page 35],
Hartmann [26, pages 183, 201, Figure 115b (after Müller)], Kornicker [48, page 84]. (Not Brady and Norman [55]). Holotype. USNM 152829, A-1 male.

Type Locality. Station X051, $21^{\circ} 48^{\prime} 01^{\prime \prime} \mathrm{N}, 17^{\circ} 02^{\prime} 00^{\prime \prime} \mathrm{W}$; 30 m; Western Sahara.

Distribution. Northeast Atlantic: off Western Sahara. (?) Southeast Atlantic: South Africa.

Habitat. Benthic; $30 \mathrm{~m}$; sand.

Life History and Ontogeny. A-1 male.

Comparisons. The species $R$. tridens differs from R. compressa Brady and Norman [55], in not having anterodorsal and ventral ribs and riblets on the surfaces o the valves. The carapace of $R$. tridens resembles that of the female of $R$. compressa from South Africa illustrated by Müller [59, Plate 7:1]. Müller's specimen has been put into the synonymy of $R$. tridens with a question because the first antenna illustrated by Müller [59, Plate 7:2] does not show a lateral bristle on the second segment, but this could be because the illustration is a medial view of the limb. The first, third, and fourth endites of the sixth limb illustrated by Müller [59, Plate 7:12] have a different number of bristles than do the same endites of the two specimens of $R$. tridens described herein, but this could be the result of intraspecific variability. Rutiderma tridens is easily separated from $R$. leloeuffi by the absence of ribs on the anterior half of the valves and by having a smaller caudal process. Klie ([5, page 406]) did not describe the specimens from Lüderitz Bay, South-West Africa, that he identified as R. compressa Brady and Norman; their identification should be verified. The carapace of $R$. tridens differs from that of $R$. mollita Darby [74], in having the caudal process projecting past the posterior end of the shell (Kornicker and Caraion [47, pages 65-66]).

5.36. Rutiderma tryx Kornicker [50] (Figure 9(d)). Rutiderma tryx. Kornicker [50, pages 31-36, Figures 19-21, Table 1, Appendices 1-2].

Holotype. QM W20741, undissected, ovigerous female, Queensland Museum.

Type Locality. Lizard Island, Australia, station AC-LI-2, west of Lizard Island Research Station, off Casuarina Beach; 200-300 m off shore of sandy beach; depth of water about $1.5 \mathrm{~m}$; substrate silty sand, some substrate very thin on flat rock lying between coral heads in patch reef.

Distribution. Collected only at type locality.

Habitat. Silty sand.

Life History and Ontogeny. Ovigerous female with 2 eggs.

Comparisons. The carapace of $R$. tryx differs from that of $R$. sagax (only male known) in having an alar process with a convex rather than a concave posterior edge. The furca of $R$. tryx bears 6 claws on each lamella compared to 7 on $R$. sagax. The anterior ridge of the infold of the left valve caudal process of $R$. dux bears numerous flat pointed spines that are absent on $R$. $\operatorname{tryx}$ (spines usually are visible through shell). The furca of $R$. $d u x$ bears 4 primary and 2 secondary claws on each lamella compared to 3 primary and 3 secondary claws on the furca of R. $\operatorname{tryx}$ (Kornicker [50, page 36]).

5.37. Rutiderma vox Kornicker [48] (Figures 9(e)-9(f)). Rutiderma sp. Kornicker [92, pages 217, 218, Figure 1b]. 


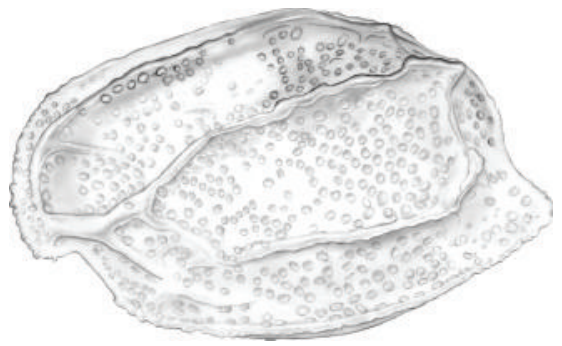

(a)

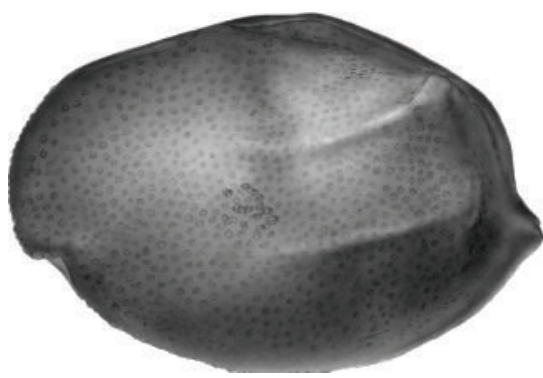

(c)

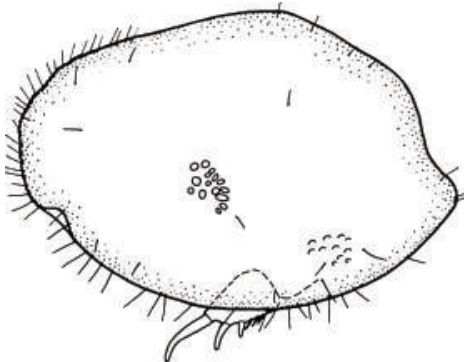

(b)

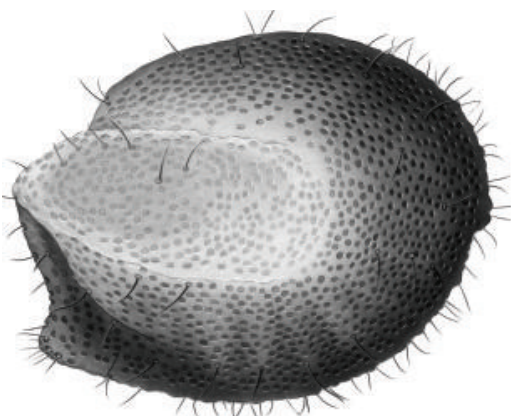

(d)

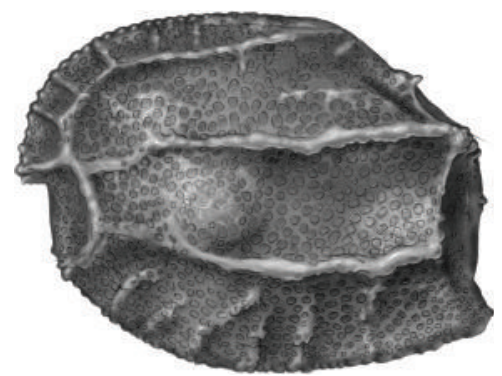

(e)

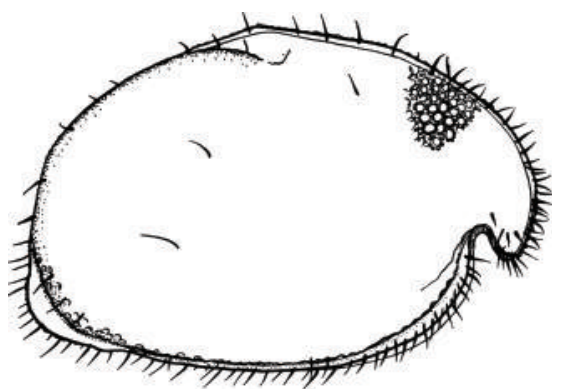

(g)

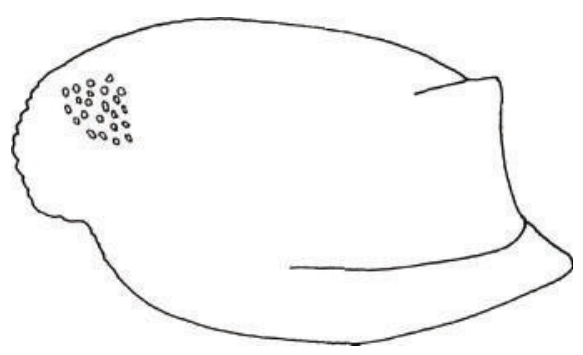

(f)

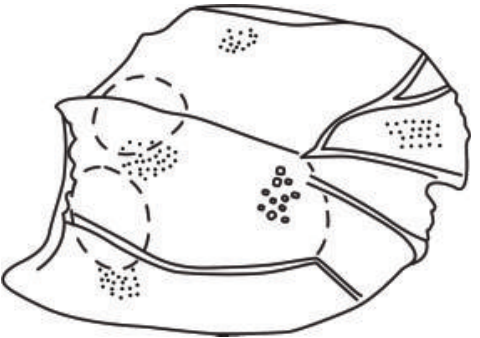

(h)

Figure 9: (a) Rutiderma. sterreri, male, $0.95 \mathrm{~mm}$. (b) R. tridens, A-1 female, $1.24 \mathrm{~mm}$. (c) R. tridens, A-1 male, $1.32 \mathrm{~mm}$. (d) R. tryx, ovigerous female, $1.10 \mathrm{~mm}$. (e) R. vox, female, $0.91 \mathrm{~mm}$. (f) R. vox, male, $1.0 \mathrm{~mm}$. (g) Scleraner chacaoi, female, $1.30-1.4 \mathrm{~mm}$. (h) S. trifax, ovigerous female, $1.77 \mathrm{~mm}$.

Rutiderma vox Kornicker [48, pages 78-84, Figures 3942, Table 1], Kornicker [37, page 114], Kornicker and Harrison-Nelson [43, Tables 8, 9].

Holotype. USNM 158309, ovigerous female.

Type Locality. Enewetak lagoon from sample collected from various airlift and emergence trap samples, from 5 to $8 \mathrm{~m}$ of water.

Distribution. Enewetak Atoll.
Habitat. Sandy lagoon shelf.

Life History and Ontogeny. Adult male, female, ovigerous female (from 1 to 4 eggs).

Comparisons. $R$. vox differs from $R$. normani in not having a serrate list on the infold of the caudal process of the right valve and from $R$. darbyi Kornicker $[16,19]$ in not having a serrate list on the infold of the caudal process of the left valve. The female $R$. vox differs from the female $R$. lomae 


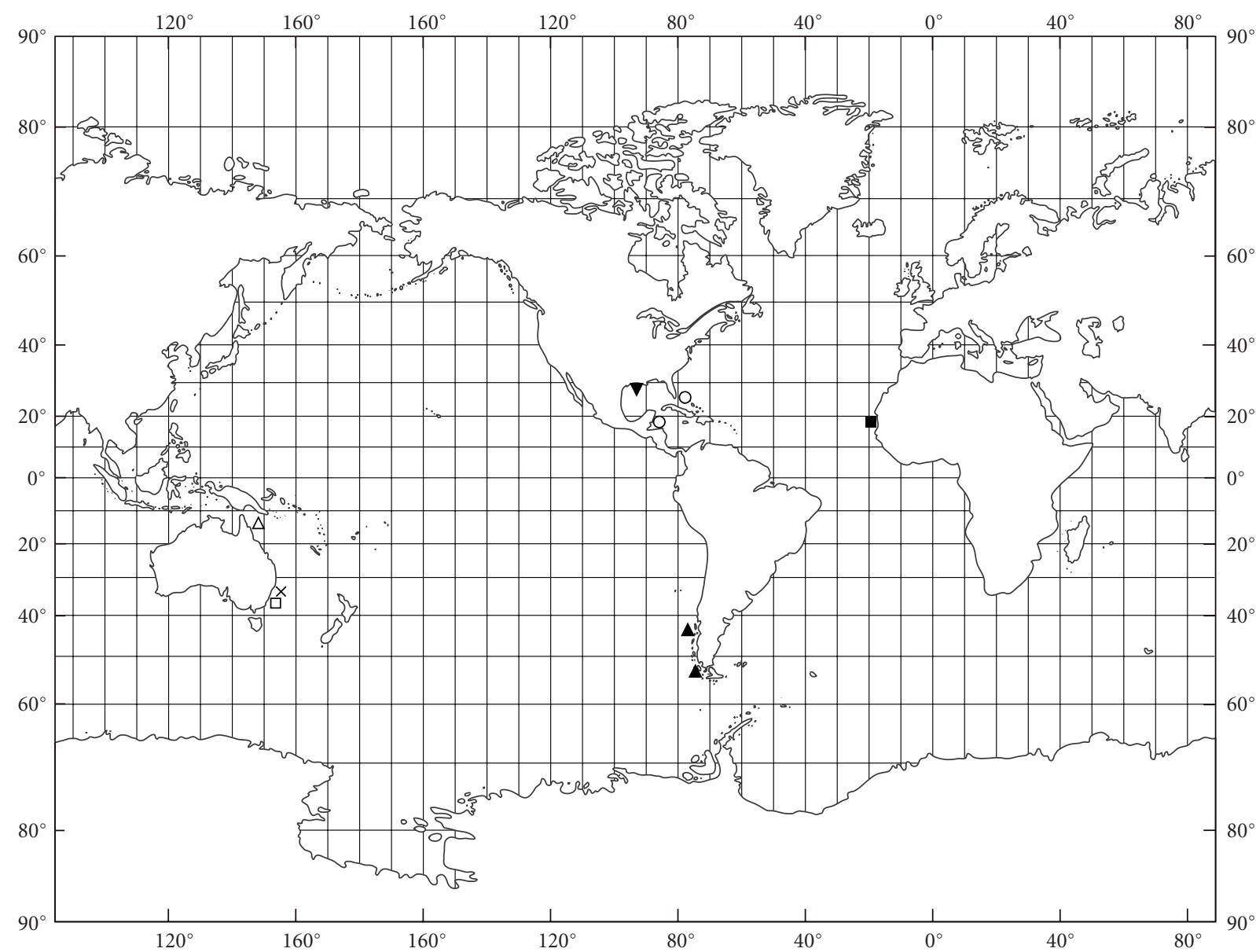

\footnotetext{
Alternochelata

$\triangle$ A. lizardensis

- A. nealei

○ A.polychelata

$\nabla$ A. sikorai
}

Scleraner

\ S. chacoi

$\times$ S. trifax

Metaschisma

․ M. nex

FIGURE 10

in having a longer caudal process, fewer bristles on the list of the caudal process, a shorter b-bristle on the seventh segment of the first antenna, an endopodite of the second antenna without a minute bristle near the middle of its margin, and a slightly stouter fourth claw on the furca. Rutiderma vox differs from $R$. judayi in not having a deep indentation at midheight of the posterior edge of the alate process on each valve. Rutiderma vox differs from $R$. gerdhartmanni, $R$. chessi, $R$. sterreri, $R$. arcuatilis, $R$. cohenae, and $R$. kalkei, in having marginal teeth on the 3 lobes of the second segment of the female fifth limb. The female $R$. vox differs from the female $R$. compressa, $R$. rostrata, $R$. leloeuffi, and $R$. tridens in not having a prolonged tip on the c-bristle of the second endopodial segment of the female mandible. Rutiderma vox differs from $R$. hartmanni, R. pax, and R. licina in not having an upturned tip on the c-bristle (claw) of the second endopodial segment of the female mandible. The list of the caudal process of the female R. vox is longer and more oblique than that of the female $R$. dinochelata.

\section{Species in Open Nomenclature}

6.1. Rutiderma species A Cohen [20]. Cohen [20, pages 322, 324, 327, 330, 331, 332, 334, 325, Figure 4].

Distribution. Belize, vicinity of Carrie Bow Key, $16^{\circ} 49^{\prime} \mathrm{N}$, $88^{\circ} 05^{\prime} \mathrm{W}$.

Habitat. Lagoon, back-reef, Thalassia, plankton; $1.5 \mathrm{~m}$. Sand and rubble zone. Outer fore-reef, $30 \mathrm{~m}$. Sand trough.

Life History and Ontogeny. Live specimens reared from egg to second instar.

6.2. Rutiderma Species KE Cohen [20]. Cohen [20, pages 324, 326].

Distribution. Belize.

Habitat. Sand trough, outer fore-reef slope, spur and groove, and back-reef.

6.3. Rutiderma Species KO Cohen [20]. Cohen [20, pages 322, 331]. 


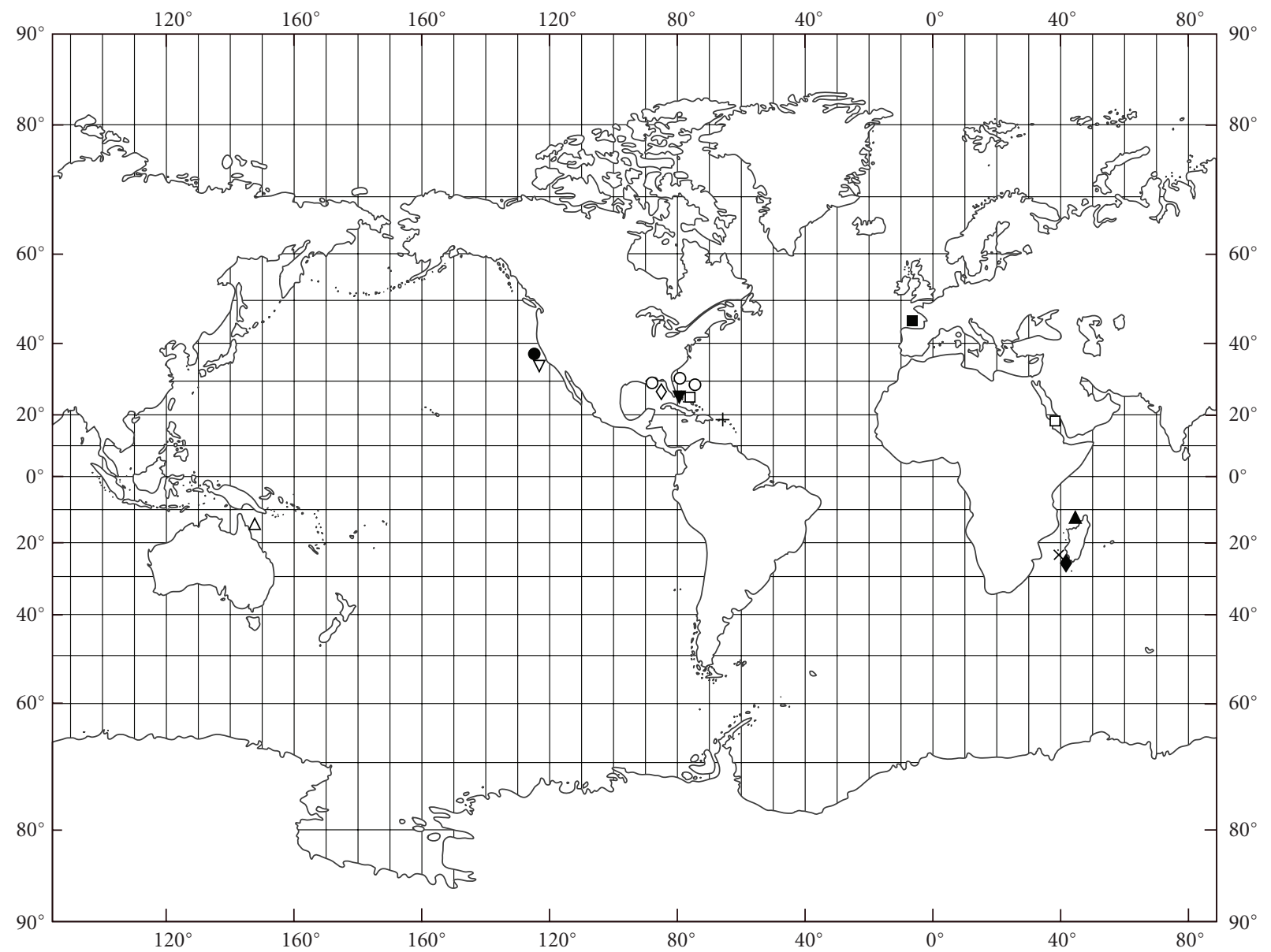

$\begin{array}{ll}\text { Rutiderma } & \\ \text { - R. apex } & \circ R . \text { darbyi } \\ + \text { R. arcuatile } & \nabla \text { R.dinochelata } \\ \Delta \text { R. arx } & \Delta R . \text { dux } \\ \nabla \text { R. chessi } & \times R . \text { exrex } \\ \diamond \text { R. cohenae } & \nabla R . \text { ferax } \\ \text { - R. compressum } & \square R . \text { flex }\end{array}$

FIgURE 11

Distribution. Belize.

Habitat. Spur and groove, lagoon plankton, outer forereef, $30 \mathrm{~m}$. Sand trough.

6.4. Rutiderma Species L Cohen [20]. Cohen [20, pages 324, 326].

Distribution. Belize.

Habitat. Sand and rubble zone, lagoon plankton.

6.5. Rutiderma sp. Spears and Abele [93]. Rutiderma sp. Spears and Abele [93, pages 172, 175 (GenBank accession number L81942)].

Remarks. This is the first study of a myodocopid using DNA sequencing.

Distribution. Near LTS buoy (fore-reef and groove), Discovery Bay Laboratory, Jamaica, $50 \mathrm{ft}$.

6.6. Scleraner Kornicker [15]. Scleraner Kornicker [13, pages 645, 646, 648 Figures 405, 656 Figures 411, 678. (Type species by original designation: Rutiderma (Rutiderma) chacaoi] Hartmann [60]. Gender: masculine, Kornicker [54, page 40 (mentions)], Kornicker [37, pages 114, 115, 123], Parker [39, page 105], Kornicker and Harrison-Nelson [43, pages $429,465,467]$.

6.7. Scleraner chacaoi [60] (Figure 9(g)). Rutiderma (Rutiderma) chacaoi Hartmann in Hartmann-Schröder and Hartmann [8, pages 328-332, Figures 36-44].

Rutiderma chacaoi. Hartmann-Schröder and Hartmann [8, pages 24, 30, 33, (station data and ecology), Table 3], Hartmann [26, page 129, Figure 73i (according to Hartmann 1959), 183, 184: Figure 73i, 106e (according to Hartmann 1965); 1975: page 68, Figure 398a (after Hartmann 1965)], Hartmann and Petersen [79, page 228 (location of types)], Kornicker and Harrision-Nelson [43, page 467, Tables 8, 9].

Scleraner chacaoi (Hartmann). Kornicker [13, pages 16, $24,29,47,60,62,70,80,650,656,678-682$, Figure 428], 

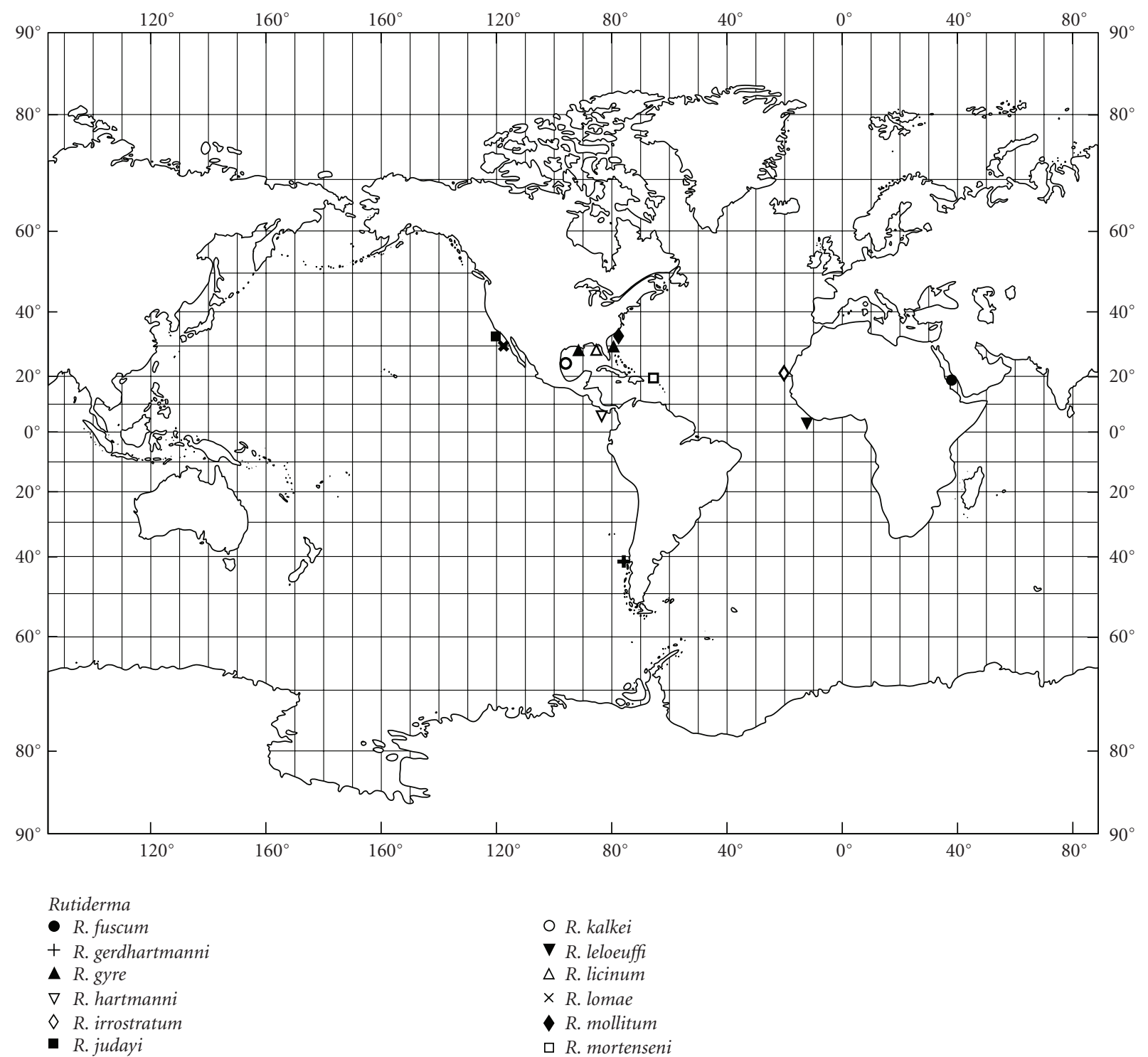

O R. kalkei

$\boldsymbol{\nabla}$ R. leloeuffi

$\triangle$ R. licinum

$\times$ R. lomae

$\checkmark$ R. mollitum

$\square$ R. mortenseni

FIGURE 12

Kornicker [16, page 12 (misspelling)], Kornicker and Cohen [29, page 8], Kornicker [37, page 123], Kornicker and Harrison-Nelson [43, page 467, Tables 8, 9].

Holotype. K 27302 A-l juvenile female, Hamburg Zoological Museum. Chile.

Type Locality. Gulf of Corcovado, Canal of Chacao, South

Distribution. Southeast Pacific: Chile: Gulf of Chacao; $53^{\circ} 41^{\prime} 40^{\prime \prime} \mathrm{S}, 72^{\circ} 0^{\prime} 45^{\prime \prime} \mathrm{W}$ (shelf), Fortescue Bay, Straits of Magellan; $50^{\circ} 16^{\prime} 42^{\prime \prime} \mathrm{S}, 74^{\circ} 48^{\prime} 28^{\prime \prime} \mathrm{W}$ (shelf).

Habitat. Benthic; $190 \mathrm{~m}$; fine-grained sand with mud, detritus and rocks, temperature about $11^{\circ} \mathrm{C}, \mathrm{O}_{2}$ about $3.5 \mathrm{ml} / \mathrm{l}, 21.3$ and $30 \mathrm{~m}$, bottom coarse with much red algae and bottom of pea gravel with mud. niles.

Life History and Ontogeny. A-1 female, A-1 male, juve-
6.8. Scleraner trifax Kornicker [37] (Figure 9(h)). Scleraner trifax Kornicker [37, pages 115-123, 192, Tables, 1, 2, Figures 64e, 65-68, Appendices 1, 2], Parker [39, Figure J], Kornicker and Harrison-Nelson [43, page 467, Tables 1, 8, 9].

Holotype. Ovigerous female in the collection of the Museum of Victoria, Australia.

Type Locality. Slope $1,34^{\circ} 59.52^{\prime} \mathrm{S}, 151^{\circ} 5.94^{\prime} \mathrm{E}$, off Nowra, New South Wales, $204 \mathrm{~m}$.

Distribution. Type locality and Slopes 21, 22, 48, 204$400 \mathrm{~m}$.

Habitat. Coarse shell, muddy shell.

Life History and Ontogeny. Adult male and female, A1 male, juveniles, from 2 to 3 eggs.

Comparisons. The carapace of $S$. trifax differs from that of $S$. chacaoi in having lateral ribs. The second segment of the endopodite of the female second antenna differs in having 1 instead of 2 bristles. Each lamella of the furca of S. trifax 


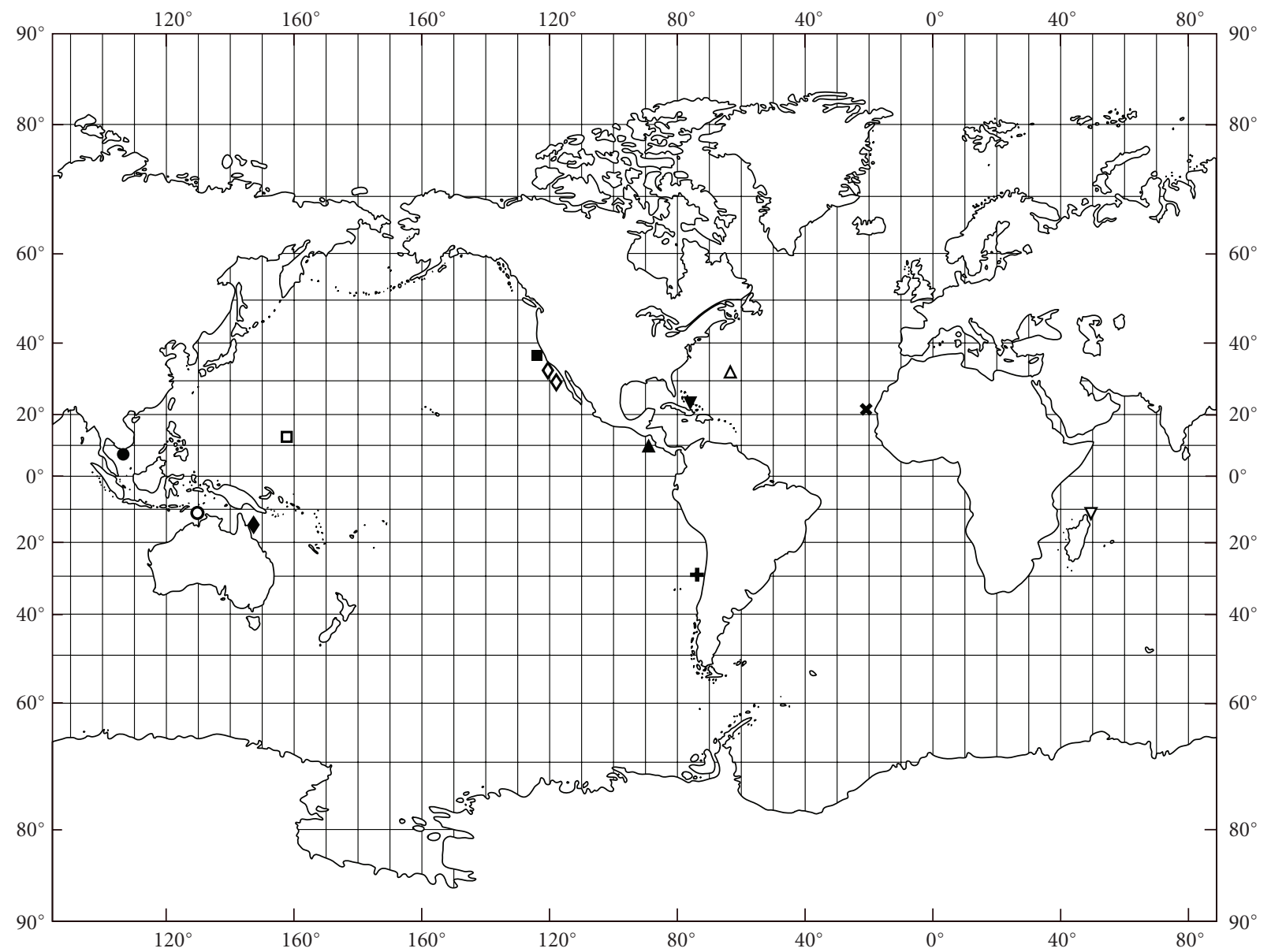

Rutiderma
- $R$. normani
+ R. ovata
\ $R$. pax
$\nabla$ R. rex
$\diamond$ R. rostratum
- $R$. rotundum

O R. sagax

$\boldsymbol{\nabla}$ R. schroederi

$\triangle$ R. sterreri

$\times R$. tridens

$\checkmark$ R. $\operatorname{tryx}$

R. vox

Figure 13

has 3 primary claws followed by 3 secondary claws; that of $S$. chacaoi has 4 primary claws followed by 5 secondary (Kornicker [37, page 123]).

\section{Acknowledgments}

The authors thank Abbie Yorkoff, Smithsonian Institution, for her careful work inchecking the literature citations and formatting the paper. They also thank Molly Kelly Ryan, formerly of the Smithsonian, for preparing the original versions of Figures 10-13.

\section{References}

[1] A. C. Cohen and L. S. Kornicker, "Catalog of the rutidermatidae (Crustacea: Ostracoda)," Smithsonian Contributions to Zoology, vol. 449, pp. 1-11, 1987.

[2] A. C. Cohen and J. G. Morin, "Sexual morphology, reproduction and the evolution of bioluminescence in Ostracoda," Paleontological Society Papers, vol. 9, pp. 37-70, 2003.
[3] A. C. Cohen, D. E. Peterson, and R. F. Maddocks, "Ostracoda," in The Light and Smith Manual: Intertidal Invertebrates from Central California to Oregon, J. T. Carlton, Ed., pp. 417446, University of California Press, Berkeley, Calif, USA, 4th edition, 2007.

[4] L. S. Kornicker, "Antarctic and Subantarctic Myodocopina (Ostracoda)," in Synopses of the Antarctic Benthos, J. W. Wagele and J. Sieg, Eds., vol. 5, p. 185, Koeltz Scientific Books, Koenigstein, Germany, 1993.

[5] W. Klie, "Beiträge zur Fauna des Eulitorals von Deutsch-Südwest-Afrika-II: ostracoden von der Küste Deutsch-SüdwestAfrikas," Kieler Meeresforschungen, vol. 3, pp. 404-448, 1940.

[6] L. S. Kornicker, "Ecology and taxonomy of Recent marine ostracodes in the Bimini Area, Great Bahama Bank," Publications of the Institute of Marine Science, vol. 5, pp. 194-300, 1958.

[7] G. Hartmann, "Zur Kenntnis der Ostracoden des Roten Meeres. (Ergebnisse Nr. 11 der Reise von A. Remane und E. Schulz nach dem Roten Meer)," Kieler Meeresforschungen, vol. 20, pp. 35-127, 1964. 
[8] G. Hartmann-Schröder and G. Hartmann, Teil 1. Beschreibung der Lebensräume und Ökologie. in Zur Kenntnis des Sublitorals der chilenischen Küste unter besonderer Berücksichtigung der Polychaeten und Ostracoden (Mit Bemerkungen über den Einfluss sauerstoffarmer Strömungen auf die Besiedlung von marinen Sedimenten). Mitteilungen aus dem Hamburgishcen Zoologishchen Museum und Institut vol. 62, supplement, pp. 1-384.

[9] K. G. McKenzie, "Myodocopid Ostracoda (Cypridinacea) from Scammon Lagoon, Baja California, Mexico, and their ecologic associations," Crustaceana, vol. 9, pp. 57-70, 1965.

[10] E. M. Poulsen, "Ostracoda-myodocopa-2: cypridiniformesrutidermatidae, sarsiellidae and asteropidae," Dana Report, vol. 65, pp. 1-484, 1965.

[11] U. Lie, "A quantitative study of benthic infauna in Puget Sound, Washington, USA, in 1963-1964," Fiskeridirektoratets Skrifter. Serie Havundersøkelser, vol. 14, pp. 229-556, 1968.

[12] U. Lie and D. S. Kisker, "Species composition and structure of benthic infauna communities off the coast of Washington," Journal of the Fisheries Research Bulletin of Canada, vol. 27, pp. 2273-2285, 1970.

[13] L. S. Kornicker, "Antarctic Ostracoda (Myodocopina) Parts 1 and 2," Smithsonian Contributions to Zoology, vol. 163, pp. 1720, 1975.

[14] L. S. Kornicker and B. Meyers, "Rutidermatidae of Southern California (Ostracoda: Myodocopina)," Smithsonian Contributions to Zoology, vol. 334, pp. 1-35, 1981.

[15] L. S. Kornicker, "Alternochelata lizardensis, a new species of myodocopine Ostracode from the Great Barrier Reef of Australia (Rutidermatidae)," Proceedings of the Biological Society of Washington, vol. 95, pp. 793-806, 1982.

[16] L. S. Kornicker, "Rutidermatidae of the continental shelf of southeastern North America and the Gulf of Mexico (Ostracoda: Myodocopina)," Smithsonian Contributions to Zoology, vol. 371, pp. 1-89, 1983.

[17] S. A. Grabe, C. C. Gibson, and R. J. Watkins, "Ecological relationships of myodocopid ostracods in the vicinity of Marco Island, Florida," Bulletin of Marine Science, vol. 56, no. 2, pp. 695-701, 1995.

[18] S. A. Grabe, "Distribution of myodocopid ostracods in Tampa Bay, Florida, and association with abiotic variables," Gulf and Caribbean Research, vol. 17, pp. 57-68, 2005.

[19] L. S. Kornicker, "The ostracode family Cypridinidae and the Genus Pterocypridina," Smithsonian Contributions to Zoology, vol. 379, pp. 1-29, 1983.

[20] A. C. Cohen, "Comparison of myodocopid ostracodes in two zones of the Belize Barrier Reef near Carrie Bow Cay with changes in distribution 1978-1981," Bulletin of Marine Science, vol. 45, no. 2, pp. 316-337, 1989.

[21] A. C. Cohen and J. G. Morin, "Patterns of reproduction in ostracodes: a review," Journal of Crustacean Biology, vol. 10, pp. 184-211, 1990.

[22] J. G. H. Horsley, A phylogenetic and ecological analysis of the Myodocopina (Myodocopa: Ostracoda) of the western continental shelf of Florida, Ph.D. Dissertation, George Washington University, Washington, DC, USA, 1990.

[23] T. Skogsberg, "Studies on marine ostracods-I: cypridinids, halocyprids, and polycopids," Zoologiska Bidrag från Uppsala, vol. 1, supplement, pp. 1-784, 1920.

[24] E. Mertens, "Zur Kenntnis der Ordnung Ostracoda (Crustacea)," Geologische Jahrbuch, vol. 75, pp. 311-318, 1958.

[25] E. M. Poulsen, "Ostracoda-Myodocopa-1: cypridiniformescypridinidae," Dana Report, vol. 57, pp. 1-414, 1962.
[26] G. Hartmann, "Ostracoda," in Klassen und Ordnungen des Tierreichs, H. G. Bronns, Ed., Buch 2, Teil 4, pp. 1-216, 1966.

[27] G. Hartmann and H. S. Puri, "Summary of neontological and paleontological classification of Ostracoda," Mitteilungen aus dem Hamburgishcen Zoologishchen Museum und Institut, vol. 70, pp. 7-73, 1974.

[28] G. Hartmann, "Ostracoda," in Klassen und Ordungen des Tierreichs, H. G. Bronns, Ed., Buch 2, Teil 4, pp. 569-786, 1975.

[29] L. S. Kornicker and A. C. Cohen, "Dantyinae, a new subfamily of Ostracoda (Myodocopina: Sarsiellidae)," Proceedings of the Biological Society of Washington, vol. 91, pp. 490-508, 1978.

[30] L. S. Kornicker, "Revision, distribution, ecology and ontogeny of the ostracode subfamily Cyclasteropinae (Myodocopina: Cylindroleberididae)," Smithsonian Contributions to Zoology, vol. 319, pp. 1-548, 1981.

[31] L. S. Kornicker, "Benthic marine Cypridinoidea from Bermuda (Ostracoda)," Smithsonian Contributions to Zoology, vol. 331, pp. 1-15, 1981.

[32] L. S. Kornicker, "Sarsiellidae of the western Atlantic and northern Gulf of Mexico, and revision of the Sarsiellinae (Ostracoda: Myodocopina)," Smithsonian Contributions to Zoology, vol. 415, pp. 1-217, 1986.

[33] L. S. Kornicker, "Cylindroleberidae of the western north Atlantic and northern gulf of Mexico, and Zoogeography of the Myodocopina (Ostracoda)," Smithsonian Contributions to Zoology, vol. 425, pp. 1-139, 1986.

[34] R. F. Maddocks and L. S. Kornicker, "Class Ostracoda (Mussel shrimps)," in Marine Fauna and Flora of Bermuda, W. Sterrer, Ed., pp. 280-288, John Wiley \& Sons, New York, NY, USA, 1986.

[35] G. Hartmann and H. E. E. Petersen, Verzeichnis der Typen aus der Sammlung Crustacea des Zoologischen Instituts und Zoologischen Museums der Universität Hamburg Klasse Ostracoda Latreille, 1806 Unterklasse Myodocopa Sars, 1866 Ordnung Myodocopida Sars, 1866. aus dem Hamburgishcen Zoologishchen Museum und Institut vol. 84, pp. 157-160, 1987.

[36] J. G. Morin and A. C. Cohen, "ONE. Bioluminescent displays, courtship, and reproduction in stracodes," in Crustacean Sexual Biology, R. T. Bauer and J. W. Martin, Eds., pp. 1-16, Columbia University Press, New York, NY, USA, 1991.

[37] L. S. Kornicker, "Ostracoda (Myodocopina) of the SE Australian continental slope-part 1," Smithsonian Contributions to Zoology, no. 553, pp. 1-200, 1994.

[38] A. R. Parker, "Functional morphology of the myodocopine (Ostracoda) furca and sclerotized body plate," Journal of Crustacean Biology, vol. 17, no. 4, pp. 632-653, 1997.

[39] A. R. Parker, "Exoskeleton, distribution, and movement of the flexible setules on the myodocopine (Ostracoda: Myodocopa) first antenna," Journal of Crustacean Biology, vol. 18, no. 1, pp. 95-110, 1998.

[40] L. S. Kornicker, "Exopod and protopodial endite III of the maxillae of Sarsiellinae (Crustacea: Ostracoda: Myodocopa)," Proceedings of the Biological Society of Washington, vol. 114, pp. 680-685, 2001.

[41] L. S. Kornicker, "Orientation of the maxilla of the Sarsiellinae (Ostracoda)," Journal of Crustacean Biology, vol. 22, no. 2, pp. 268-278, 2002.

[42] L. S. Kornicker, "Comparative morphology of the fifth limb (second maxilla) of Myodocopid Ostracoda," Journal of Crustacean Biology, vol. 22, no. 4, pp. 798-818, 2002. 
[43] L. S. Kornicker and E. Harrison-Nelson, "Ontogeny of Rutiderma darbyi (Crustacea: Ostracoda: Myodocopida: Rutidermatidae) and comparisons with other Myodocopina," Proceedings of the Biological Society of Washington, vol. 115, no. 2, pp. 426-471, 2002.

[44] L. S. Kornicker, T. M. Iliffe, and E. Harrison-Nelson, "Ostracoda (Myodocopa) from Bahamian blue holes," Smithsonian Contributions to Zoology, vol. 616, pp. 1-97, 2002.

[45] K. Frame, G. Hunt, and K. Roy, "Intertidal meiofaunal biodiversity with respect to different algal habitats: a test using phytal ostracodes from Southern California," Hydrobiologia, vol. 586, no. 1, pp. 331-342, 2007.

[46] G. W. Müller, “Ostracoda," Das Tierreich, vol. 31, pp. 1-434, 1912.

[47] L. S. Kornicker and F. E. Caraion, "West African myodocopid Ostracoda (Sarsiellidae, Rutidermatidae)," Smithsonian Contributions to Zoology, vol. 250, pp. 1-110, 1978.

[48] L. S. Kornicker, "Myodocopid Ostracoda of Enewetak and Bikini Atolls," Smithsonian Contributions to Zoology, vol. 505, pp. 1-140, 1991.

[49] L. S. Kornicker, "Myodocopid Ostracoda of the Benthédi Expedition, 1977, to the NE Mozambique Channel, Indian Ocean," Smithsonian Contributions to Zoology, vol. 531, pp. 1243, 1992.

[50] L. S. Kornicker, "Ostracoda (Myodocopina) from shallow waters of the Northern Territory and Queensland, Australia," Smithsonian Contributions to Zoology, vol. 578, pp. 1-97, 1996.

[51] L. S. Kornicker and E. Harrison-Nelson, "Myodocopid Ostracoda of Pillar Point Harbor, Half Moon Bay, California," Smithsonian Contributions to Zoology, vol. 593, pp. 1-53, 1997.

[52] L. S. Kornicker and B. A. Thomassin, "Ostracoda (Myodocopina) of the Tuléar Reef Complex, SW Madagascar," Smithsonian Contributions to Zoology, vol. 595, pp. 1-134, 1998.

[53] E. Harrison-Nelson and L. S. Kornicker, "Myodocopa Ostracoda (Crustacea) of the Gulf of Mexico," in Gulf of Mexico, Origin, Waters, and Biota, D. L. Felder and D. K. Camp, Eds., vol. 1 of Biodiversity, pp. 871-875, Texas A \& M University Press, College Station, Tex, USA, 2009.

[54] L. S. Kornicker, "Ivory Coast Ostracoda (Suborder Myodocopina)," Smithsonian Contributions to Zoology, vol. 197, pp. 1-46, 1975.

[55] G. S. Brady and A. M. Norman, "A monograph of the marine and fresh water Ostracoda of the North Atlantic and of Northwestern Europe," The Scientific Transactions of the Royal Dublin Society, vol. 5, pp. 621-784, 1896.

[56] L. S. Kornicker, T. M. Iliffe, and E. Harrison-Nelson, "Ostracoda (Myodocopa) from anchialine caves and ocean blue holes," Zootaxa, no. 1565, pp. 1-151, 2007.

[57] W. B. Flint, "Gulf of Mexico outer continental shelf benthos: macroinfaunal-environmental relationships," Biological Oceanography, vol. 1, pp. 135-155, 1981.

[58] C. Juday, "Ostracoda of the San Diego region-II: littoral forms," University of California Publications in Zoology, vol. 3, pp. 135-156, 1907.

[59] G. W. Müller, "Ostracoden der deutschen südpolar-expedition 1901-1903," Zoologie, vol. 10, pp. 51-181, 1908.

[60] G. Hartmann, "Zur Kenntnis des Sublitorals der chilenischen Küste unter besonderer Berücksichtigung der Polychaeten und Ostracoden (Mit Bermerkungen über den Einfluss," in Teil III. Ostracodens des Sublitorals, G. Hartmann-Schröder and G. Hartmann, Eds., vol. 62, pp. 1-384, Mitteilungen aus dem Hamburgishcen Zoologischen Museum und Institut, 1965.
[61] G. Bonaduce, B. Ciliberto, G. Minichelii, M. Masoli, and N. Pugliese, "The Red Sea benthic ostracodes and their geographical distribution," in Proceedings of the 8th International Symposium on Ostracoda: Applications of Ostracoda, R. F. Maddocks, Ed., pp. 472-491, Department of Geosciences, University of Houston, 1982.

[62] J. C. McCain, "Marine ecology of Saudi Arabia the nearshore, soft-bottom benthic communities of the northern area, Arabian Gulf, Saudi Arabia," Fauna of Saudi Arabia, vol. 6, pp. 79-101, 1984.

[63] L. S. Kornicker, "Sexual dimorphism, ontogeny, and functional morphology of Rutiderma hartmanni Poulsen, 1965 (Crustacea: Ostracoda)," Smithsonian Contributions to Zoology, vol. 408, pp. 1-28, 1985.

[64] T. H. Oakley and C. W. Cunningham, "Molecular phylogenetic evidence for the independent evolutionary origin of an arthropod compound eye," Proceedings of the National Academy of Sciences of the United States of America, vol. 99, no. 3, pp. 14261430, 2002.

[65] T. H. Oakley, "Myodocopa (Crustacea: Ostracoda) as models for evolutionary studies of light and vision: multiple origins of bioluminescence and extreme sexual dimorphism," Hydrobiologia, vol. 538, no. 1-3, pp. 179-192, 2005.

[66] M. D. Tuel, M. P. Wilderman, J. E. Trapani, and P. E. Hayes, A biological survey of Pillar Point Harbor, El Granada, California. Report prepared by Marine Ecological Institute, Redwood City, California, for San Mateo County Harbor District, Pillar Point Harbor, El Granda, California, 1976.

[67] T. H. Oakley, "On homology of arthropod compound eyes," Integrative and Comparative Biology, vol. 43, no. 4, pp. 522530, 2003.

[68] E. K. Kempf, Index and bibliography of marine Ostracoda, Geologishches Institut, Universität zu Köln, Köln, Germany, 1986.

[69] H. S. Puri, "Ecologic distribution of recent Ostracoda," in Proceedings of the Symposium on Crustacea-part 1. Marine Biological Association of India Symposium Series, vol. 2, pp. 457-495, 1966.

[70] G. Hartmann, "Zur Kenntnis des Eulitorals der afrikanischenWestküste zwischen Angola und Kap der Guten Hoffnung und der afrikanischen Ostküste von Südafrika und Moçambique unter besonderer Berücksichtigung der Polychaeten und Ostracoden," in Teil III. Die Ostracoden des Untersuchungsgebiets, vol. 69, pp. 229-520, Mitteilungen aus dem Hamburgischen Zoologischen Museum und Institut, 1974.

[71] L. S. Kornicker and T. M. Iliffe, "Ostracoda (Myodocopina, Cladocopina, Halocypridina) mainly from Anchialine Caves in Bermuda," Smithsonian Contributions to Zoology, vol. 475, pp. 1-88, 1989.

[72] G. Hartmann-Schröder and G. Hartmann, Zur Kenntnis des Eulitorals der afrikanischenWestküste zwischen Angola und Kap der Guten Hoffnung und der afrikanishchen Ostküste von Südafrika und Moçambique unter besonderer Berücksichtigung der Polychaeten und Ostracoden. Teil 1. Beschreibung der Lebensräume, Ökologie und Zoogeographie. Mitteilungen aus dem Hamburgischen Zoologischen Museum und Institut vol. 69, supplement, pp. 5-94, 1974.

[73] G. Hartmann and G. Hartmann-Schröder, "Zoogeography and biology of littoral ostracoda from South Africa, Angola and Mozambique," Bulletin of American Paleontology, vol. 65, no. 282, pp. 353-367, 1975. 
[74] D. G. Darby, "Ecology and taxonomy of Ostracoda in the vicinity of Sapelo Island, Georgia," in Four Reports of Ostracod Investigations, R. V. Kesling, Ed., pp. 1-77, University of Michigan, Ann Arbor, Mich, USA, 1965.

[75] J. M. Bradford, "First record of a copepod parasitizing the ostracod family Rutidermatidae (Myodocopina: Cypridinacea): Sphaeronella spinosa n. sp. (Copepoda: Choniostomatidae)," Proceedings of the Biological Society of Washington, vol. 93, pp. 141-144, 1980.

[76] L. S. Kornicker, "Relationship between the free and attached margins of the myodocopid ostracod shell," in The Taxonomy, Morphology and Ecology of Recent Ostracoda, J. W. Neale, Ed., pp. 109-135, Oliver and Boyd, Edinburgh, UK, 1969.

[77] L. S. Kornicker and T. M. Iliffe, "Myodocopid Ostracoda from Exuma Sound, Bahamas, and from marine caves and Blue Holes in the Bahamas, Bermuda, and Mexico," Smithsonian Contributions to Zoology, vol. 606, pp. 1-98, 2000.

[78] A. C. Cohen and L. S. Kornicker, "Taxonomic indexes to Ostracoda (Suborder Myodocopina) in Skogsberg (1920) and Poulsen (1962, 1965)," Smithsonian Contributions to Zoology, vol. 204, pp. 1-29, 1975.

[79] G. Hartmann and H. E. E. Petersen, Verzeichnis der Typen aus der Sammlung Crustacea des Zoologischen Instituts und Zoologischen Museums der Universität Hamburg Unterklasse Ostracoda Latreille, 1806 4. Ordnung Myodocopida Sars, 1866. Mitteilungen aus dem Hamburgishcen Zoologishchen Museum und Institut, vol. 75, pp. 225-234. 1978.

[80] L. S. Kornicker and A. C. Cohen, "The relative position of the left and right lamellae of the furca in the order Myodocopida (Crustacea: Ostracoda)," Proceedings of the Biological Society of Washington, vol. 94, pp. 739-744, 1981.

[81] L. S. Kornicker, "Ostracoda from the west coast of Central America (Myodocopina: Cypridinacea)," Proceedings of the Biological Society of Washington, vol. 97, pp. 127-134, 1984.

[82] G. Hartmann, "Zur Kenntnis der lotischen Lebensbereiche der pazifischen Küste von El Salvador unter besonderer Berücksichtigung seiner Ostracodenfauna-III. Beitrag zur Fauna El Salvadors," Kieler Meeresforschungen, vol. 15, pp. 187-241, 1959.

[83] V. Z. Lucas, "Some Ostracoda of the Vancouver Island region," Contributions to Canadian Biology and Fisheries, Studies from the Biological Stations of Canada, vol. 6, pp. 399-416, 1931.

[84] G. Hartmann, "Zur Kenntnis des Mangrove-Estero-Gebietes von El Salvador und seiner Ostracodes-Fauna," Keiler Meereforschungen Institut für Meereskunde der Universität Kiel, vol. 12, pp. 219-248, 1956.

[85] G. Hartmann, "Contribución al conocimiento de la región de esteros y manglares de El Salvador y su fauna de ostrácodos (I. Parte)," Universidad de El Salvador (Comunicaciones del Instituto Tropical de Investigaciones Cientificas), vol. 6, pp. 47$81,1957$.

[86] G. Hartmann-Schröder and G. Hartmann, "Zur Kenntis des Eulitorals der chilenischen Pazifikküste und der argentinischer Küste Südpatagoniens unter besonderer Berücksichtigung der Polychaeten und Ostracoden," Mitteilungen aus dem Hamburgischen Zoologischen Museum und Institut, vol. 60, supplement, pp. 1-270, 1962.

[87] G. Hartmann, "Zur Kennis des Eulitorals der chilenischen Pazifikküste und der argentinischer Küste Südpatagoniens unter besonderer Berücksichtigung der Polychaeten und Ostracoden," in Teil III. Ostracoden des Eulitorals, G. HartmannSchröder and G. Hartmann, Eds., vol. 60, pp. 1-270, Mitteilungen aus dem Hamburgischen Zoologischen Museum und Institut, 1962.
[88] E. L. Michael and G. F. McEwen, "Hydrographic, plankton, and dredging records of the Scripps Institution for Biological Research of the University of California 1901 to 1912 compiled and arranged under the supervision of W. E. Ritter," University of California Publications in Zoology, vol. 15, pp. 1-206, 1915.

[89] T. Hanai, N. Ikeya, and M. Yajima, "Checklist of Ostracoda from Southeast Asia," The University Museum, The University of Tokyo Bulletin, vol. 17, pp. 1-236, 1980.

[90] S. Hiruta, "Post-embryonic development of myodocopid Ostracoda," in Proceedings of the 8th International Symposium on Ostracoda, R. F. Maddocks, Ed., pp. 667-677, University of Houston, Texas, University Park, Tex, USA, 1983.

[91] A. R. Jones, "Sedimentary relationships and community structure of benthic crustacean assemblages of reef-associated sediments at Lizard Island, Great Barrier Reef," Coral Reefs, vol. 3, no. 2, pp. 101-111, 1984.

[92] L. S. Kornicker, "Chapter 21. Ostracoda (Myodocopina) of Enewetak Atoll," in The Natural History of Enewetak Atoll, Volume II, D. M. Devaney, E. S. Reese, B. L. Burch, and P. Helfrich, Eds., Biogeography and Systematics, p. 252, U.S. Department of Energy, Office of Scientific and Technical Information, Oak Ridge, Tenn, USA, 1987.

[93] T. Spears and L. G. Abele, "Crustacean phylogeny inferred from $18 \mathrm{~S}$ rDNA," in Arthropod Relationships, R. A. Fortey and R. H. Thomas, Eds., pp. 169-187, Chapman and Hall, London, UK, 1998. 

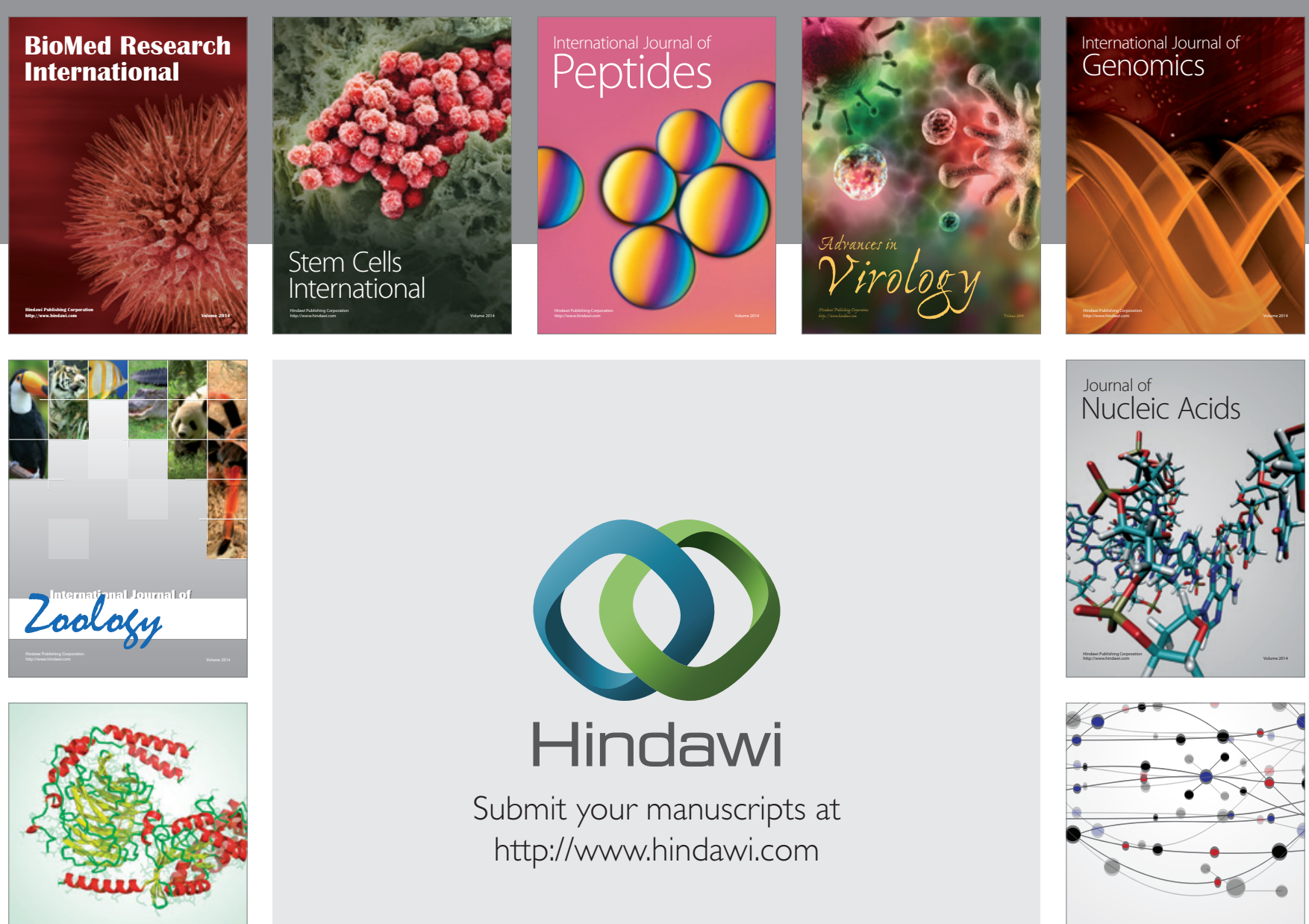

Submit your manuscripts at

http://www.hindawi.com

Signal ${ }^{\text {Jumal }}$ Transduction
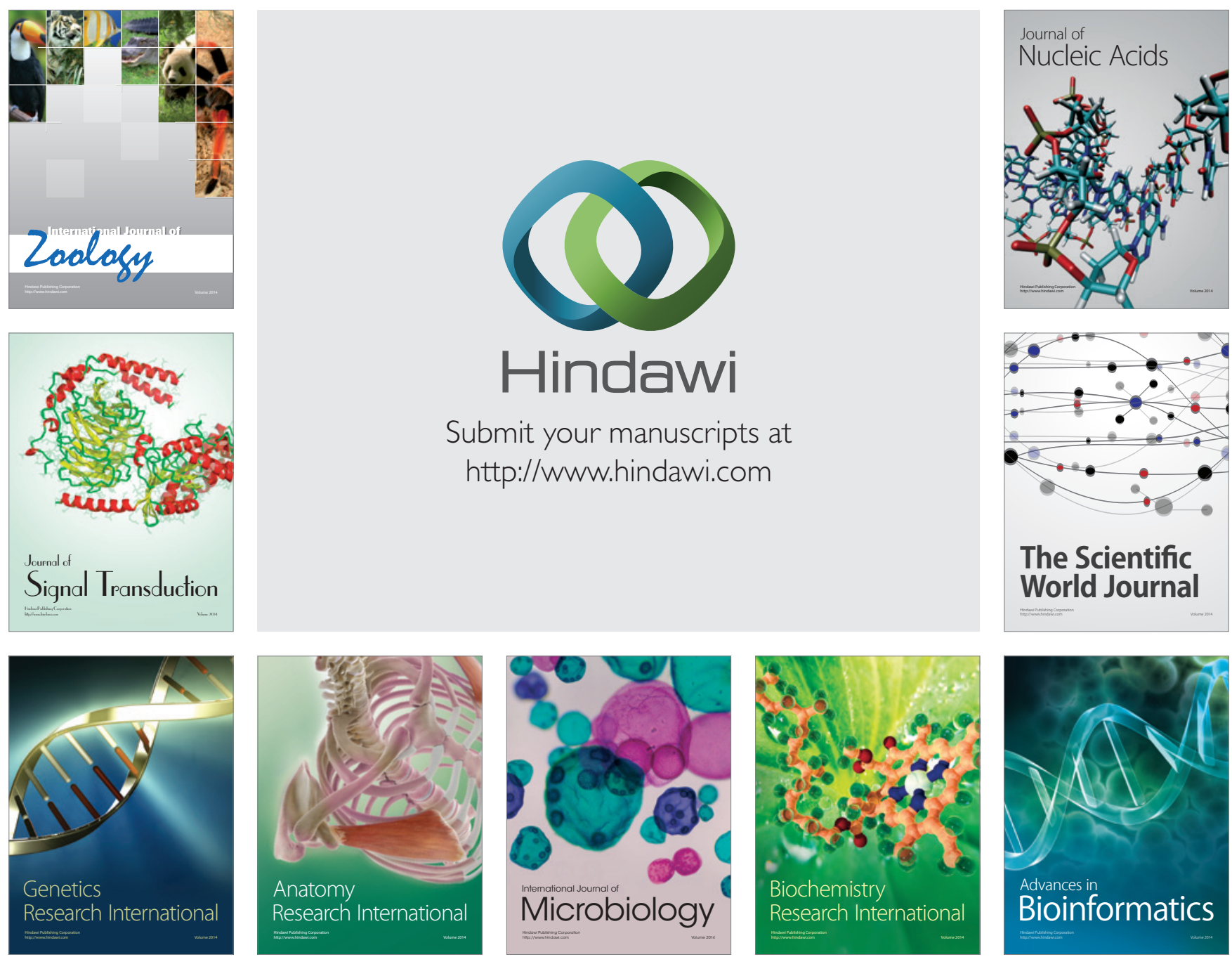

The Scientific World Journal
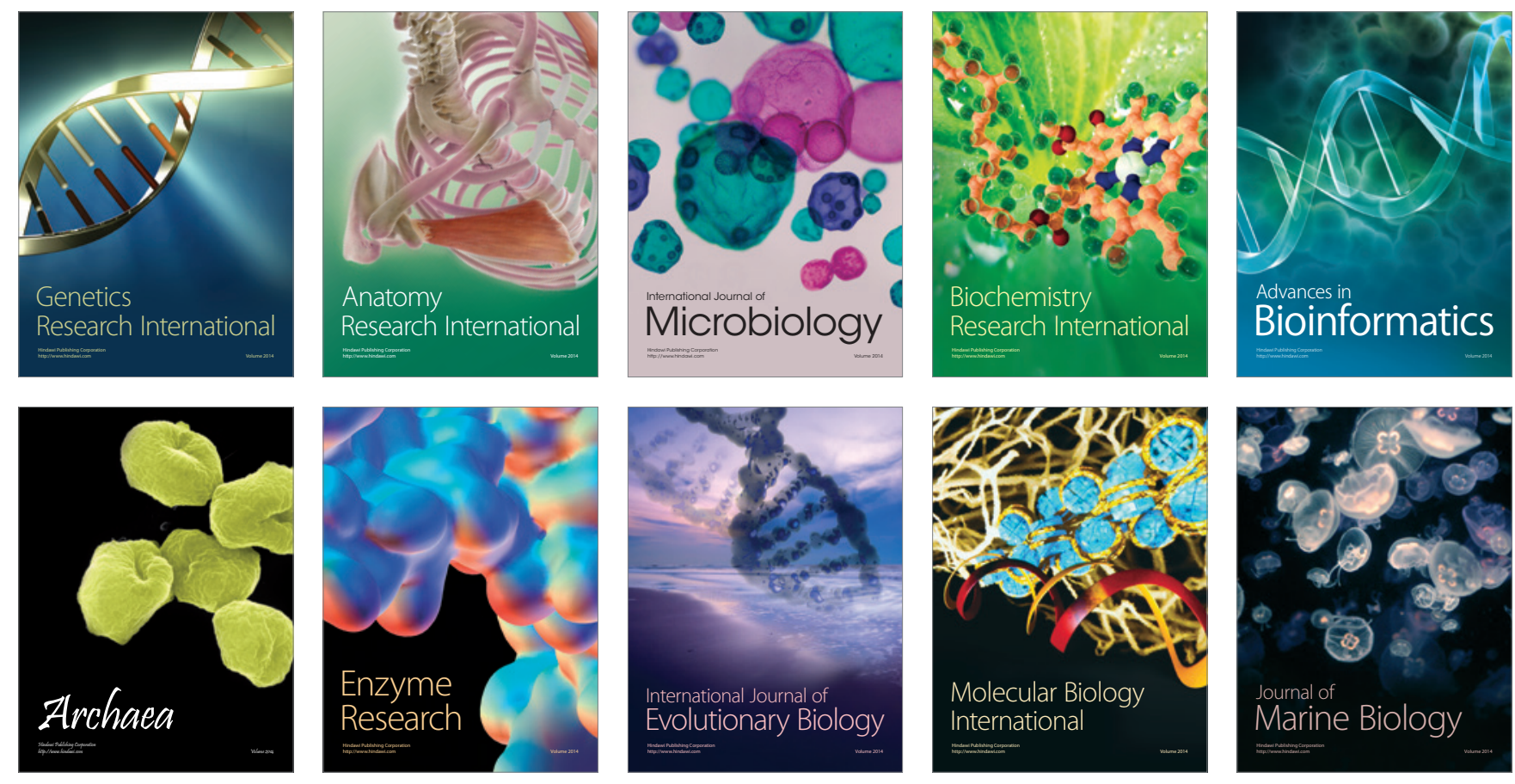